NIST GCR 15-988

\title{
On Extending an ISO Standard for Exchanging Product Manufacturing Information
}

\author{
Thomas Thurman \\ TRThurman Consulting
}

This publication is available free of charge from: https://doi.org/10.6028/NIST.GCR.15-988

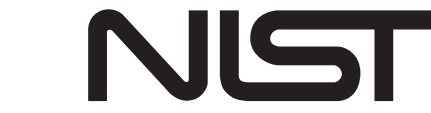

National Institute of Standards and Technology U.S. Department of Commerce 
NIST GCR 15-988

\title{
On Extending an ISO Standard for Exchanging Product Manufacturing Information
}

\author{
Prepared for \\ U.S. Department of Commerce \\ Engineering Laboratory \\ National Institute of Standards and Technology \\ Gaithersburg, MD 2089
}

By

Thomas Thurman

TRThurman Consulting

This publication is available free of charge from: https://doi.org/10.6028/NIST.GCR.15-988

February 2015

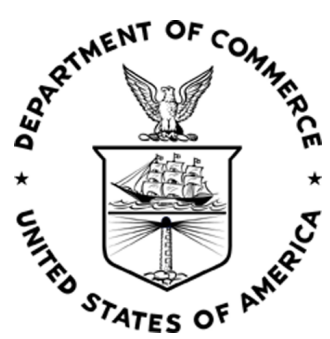

U.S. Department of Commerce Penny Pritzker, Secretary

National Institute of Standards and Technology Willie May, Acting Under Secretary of Commerce for Standards and Technology and Acting Director 


\section{NIST GCR 15-988}

Disclaimer:

This report was prepared by Thomas Thurman.

Mr. Thurman was supported under Award No. SB1341-13-SE-0287 from the National Institute of Standards and Technology (NIST), U.S. Department of

Commerce. The statements, findings, conclusions, and recommendations are those of the author and do not necessarily reflect the views of NIST or the U.S.

Department of Commerce. 


\begin{abstract}
Industrial users of ISO 10303 (STEP) protocols need the ability to exchange design product data that is in conformance with recently updated ISO Geometric Product Specifications (GPS) ISO 1101:2012; with AWS A2.4:2012 Standard Symbols for Welding, Brazing, and Nondestructive Examination; with ISO 2553: 2013 Welding and allied processes — Symbolic representation on drawings — Welded joints; with SAE AS8879D and ISO 3161:1999 Aerospace — UNJ threads - General requirements and limit dimensions; with and ISO 5855-1:1999 — Aerospace — MJ threads — Part 1: General requirements. The LOTAR consortium created a project to extend ISO 10303-242 for these new capabilities. Changes to relevant STEP information models have been proposed to support the additions to ISO 1101, and new information models proposed to support the welding and thread standards.
\end{abstract}

\title{
Keywords
}

STEP; computer aided design; data exchange; data model; dimension model; dimension representation; dimensioning and tolerancing; geometric dimensioning and tolerancing; geometrical product specification; product and manufacturing information; integration; geometric tolerance; product data; profile tolerance; tolerance model; tolerance representation; weld; welding symbol; screw thread; design context. 


\section{Introduction}

\section{Motivation}

Industrial users of STEP[1] protocols need the ability to exchange design product data definitions that are in compliance with product manufacturing information (PMI) standards. Industry needs to continue migration from drawing based PMI exchange to full model based exchange and sharing. Welding symbology and Screw thread specifications are widely used but were not addressed in ISO 10303-242:2014[2].

\section{Problem statement}

Extensions for welding symbols, screw threads and to support recent updates in Geometric Product Specifications (GPS) ISO 1101[3] are needed for [1] in a design context. AWS A2.4:2012 Standard Symbols for Welding, Brazing, and Nondestructive Examination[4] and ISO 2553: 2013 Welding and allied processes — Symbolic representation on drawings — Welded joints[5] provide source requirements for welding symbology in a design context. SAE AS8879D[6], ISO 3161:1999 Aerospace — UNJ threads — General requirements and limit dimensions[7], and ISO 5855-1:1999 — Aerospace — MJ threads — Part 1: General requirements[8] provide source requirements for screw thread properties in a design context. The LOTAR consortium created a project to extend ISO 10303-242 for these new capabilities. Changes to relevant STEP information models have been proposed to support the extensions. Upward compatibility is a constraint on any modifications to the STEP information models where there are existing implementations. 


\section{Approach}

Existing capabilities of STEP in the design context were compared to the requirements defined by the identified requirements and extensions were created as needed for the scope identified by the industrial users. The use of the EXPRESS[10] SELECT and SUBTYPE constructs in extensions to existing constructs were proposed to provide upward compatibility.

\section{Scope and contribution}

This document describes the structure of the proposed screw thread and weld information models to be added to STEPmod[11] Application Reference Model (ARM) models. It also identifies needed maintenance actions when the manufacturing Application Protocols (APs) are incorporated into STEPmod.

\section{Results}

New ARM EXPRESS models for Screw thread and for Weld are proposed. The EXPRESS models were integrated using STEPmod[11] architecture in the STEP Module and Resource Library version 6[12] into a long form EXPRESS model for evaluation. Preliminary review of mapping onto the STEP product model identified one upward compatibility risk that would be addressed by using the EXPRESS SELECT type to extend an existing model. Maintenance issues with several Application Modules (AMs) were identified. 


\section{Development process}

The information model development process used an incremental approach. The industrial user base provided coverage and implementation interpretation issues. The ASME and ISO committees provided source standard documents for the information model update and creation process. Members of the ASME and ISO committees were available for and contributed to the development process as subject matter experts. Issues that had not been dealt with by the ASME and ISO committees clearly enough were deferred for later work. There is no intent on the part of STEP developers that the interpretation of product data by receiving systems shall deviate from the requirements specified in the ASME and ISO standards, as clearly the requirement on the STEP protocol is to provide a computer interpretable representation of the relevant content of the source standards and not to develop a new or enhanced GPS system. Issues that were deemed in scope, based on resources and schedule and availability of a source standard, were analyzed by subject matter experts and STEP developers for accuracy. Requirements were identified, architectural enhancements were proposed; impact to existing implementations and data sets was considered along with capability of the architecture to support evolution of the source standards.

\section{Updates to PMI model}

\section{Screw thread}

These standards were identified as source standards based on industrial aerospace and defense requirements identified by LOTAR[13]:

SAE AS8879D[14] and ISO 3161:1999 Aerospace — UNJ threads - General requirements and limit dimensions[7]; 
ISO 5855-1:1999 —Aerospace — MJ threads — Part 1: General requirements[8].

It is recognized that this is not a general solution to exchange of thread data. In order to clearly separate the design and manufacturing contexts, a new AM Screw_thread is proposed. This AM does not provide information about the shape from which the thread is created but provides a parametric thread data set that is placed in the design geometric model. A template definition is provided to support re-use. Both Inch and Metric threads are supported, as well as catalogue and threads defined only by description. For development purposes, the schema interface provides explicit USE FROM and REFERENCE FROM to existing AMs. For development purposes, several Application Objects (AOs) are explicitly included in the thread AM that will be moved to external AMs for publication because the Screw_thread is not the correct scope for those AOs. The Screw_thread AM should be directly referenced by the implementation module ISO/TS 10303-442[15] upon publication. 


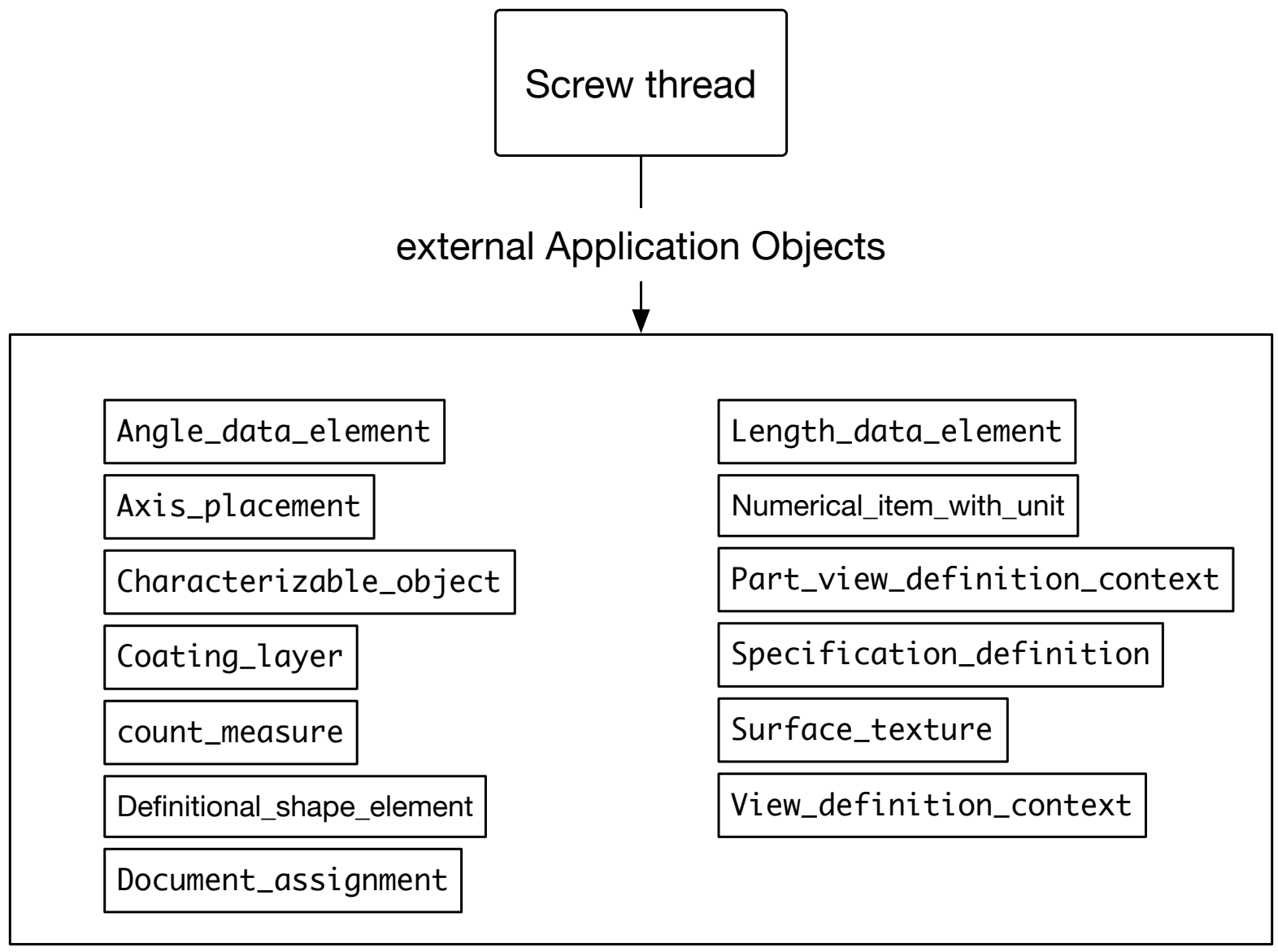

Figure 1 illustrates the external Application Objects directly referenced by the Screw_thread application module ARM EXPRESS.

The Application Objects identified in Figure 1 are formally interfaced by the EXPRESS USE FROM declaration. Those AOs define direct attributes and SUPERTYPEs of the Application Objects defined in the Screw_thread ARM EXPRESS. The AOs also bound the scope of the externally referenced AMs as applied to the screw thread design domain. For example, only an Axis_placement_shape_element is required allowing the AM Screw_thread to be completely independent of geometric model type. 


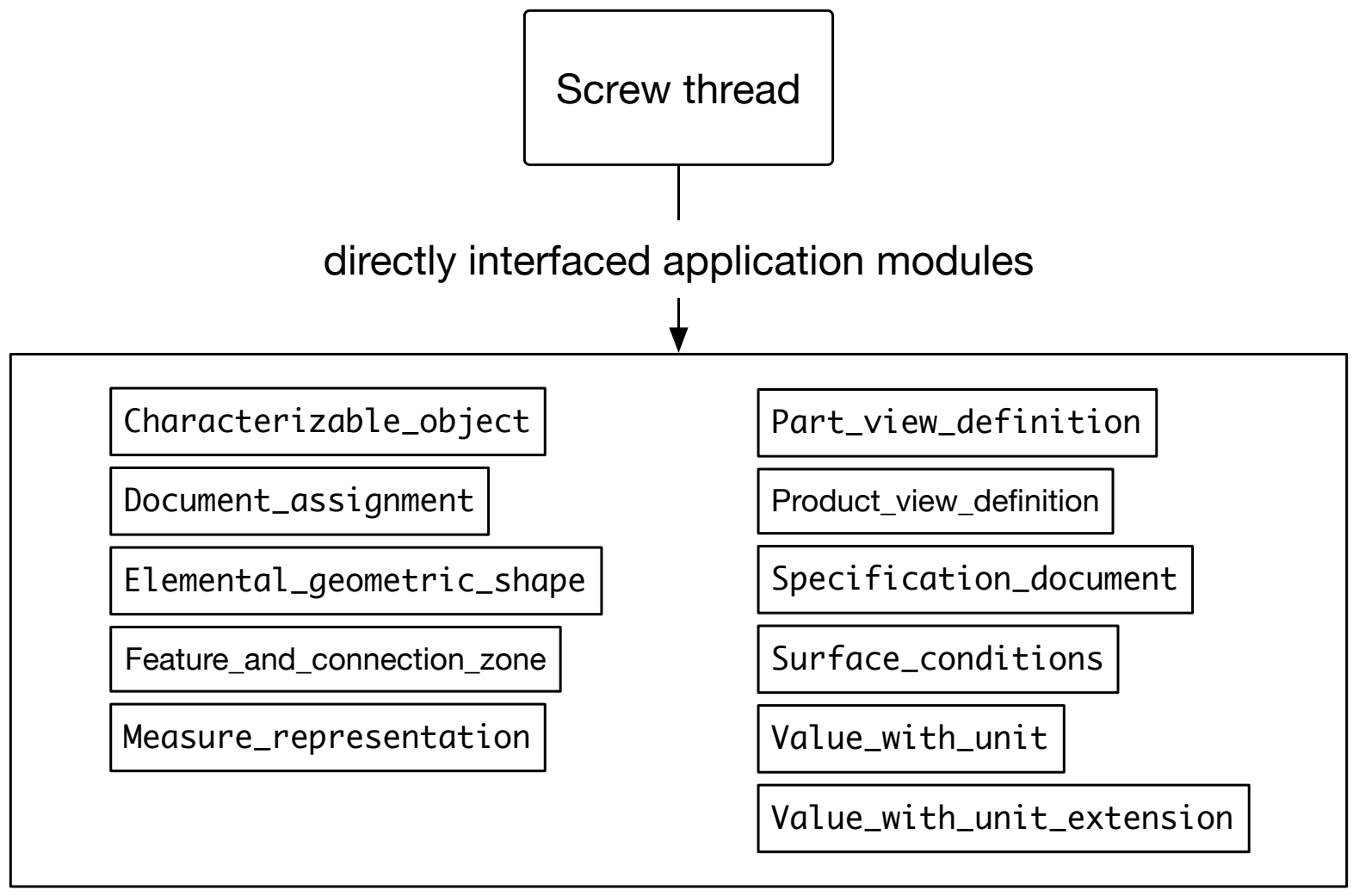

Figure 2 illustrates the external application modules that contain the directly referenced Application Objects included in Figure 1.

The Application Modules illustrated in Figure 2 are formally interfaced by the EXPRESS USE FROM declaration. 


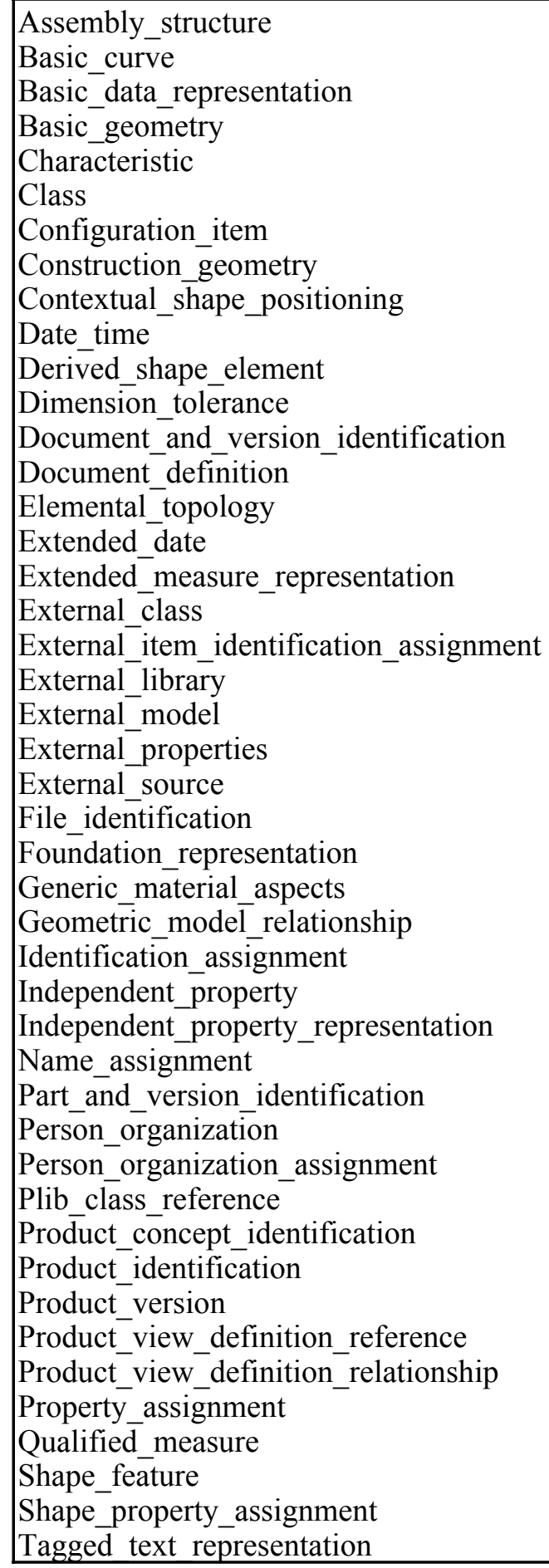

Table One identifies the Application Modules indirectly included in Screw_thread module by executing a short to long form transform on the ARM EXPRESS as defined in [10]. 
Several Application Modules in Table One are not in the scope of screw threads. The consequence is that validation and testing will be more expensive due to the added effort of tracking out of scope items. The out of scope modules should be removed from the scope during the next phase of the development process as identified in[11]. As an example, there is no requirement for AM Assembly_structure[16] to be included in the scope of screw thread design features. 


\section{Welding symbology}

Welding symbology and associated characteristics are defined in

AWS A2.4:2012 Standard Symbols for Welding, Brazing, and Nondestructive

Examination[4];

ISO 2553:2013 Welding and allied processes — Symbolic representation on drawings -

Welded joints[5].

Welded joints are defined by a welding symbol that contains a number of elements.

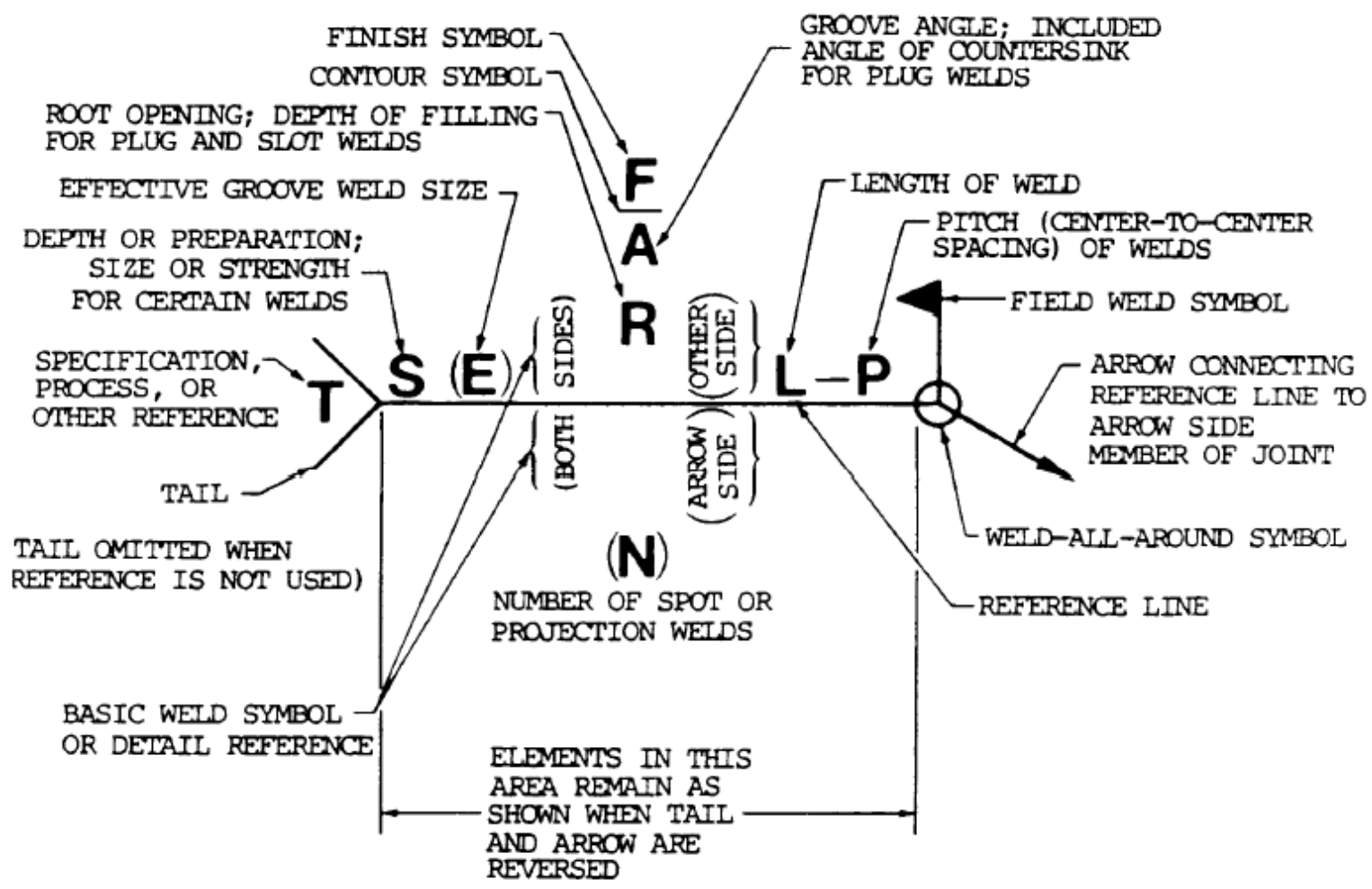

Figure 3[17] illustrates the elements of a welding symbol that is in accordance with[4].

A welding symbol associates a welded joint with the materials, process, and inspection requirements to achieve the welded joint. After review of the current capabilities of the STEP modules and resources library (SMRL) version 6[12], it was determined that a welded joint 
should be modeled as a SUBTYPE of the AO Assembled_with_bonding found in AM ISO/TS 10303-1649 Assembly_technology[18].

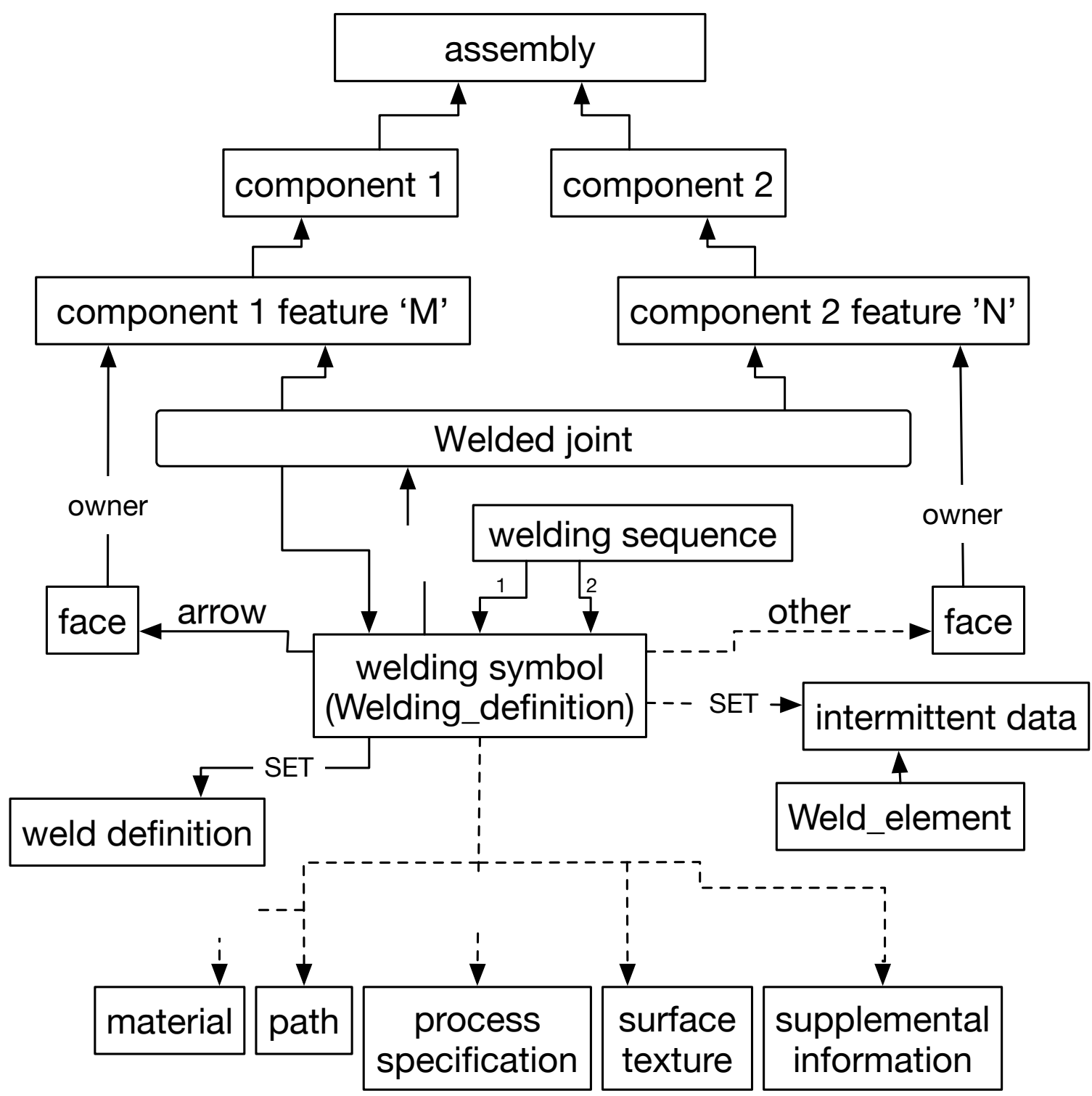

Figure 4 illustrates the key domain concepts to represent in the Weld application module.

Legend: In Figure 4 the arrows indicate dependency relations. Solid lines indicate mandatory relations. Dashed lines indicate optional relations. Cardinality of more than one is indicated by the SET keyword. 
The concepts and Application Objects identified in Figure 4 result from direct interpretation of the source documents. An assembly has two components (component 1) and (component 2). Each component has a feature of interest for welding. Each feature has at least one face specified for welding. In Figure 4 the term 'face' is a concept that is represented by a Face_shape_element[19] in the model. The face is on the surface of the component feature. In a CAD model the face would be represented by a geometric face entity that might be helping to compose the 3D model. Alternatively, the face may be placed on the surface specifically to support the welding symbol. Assembly, component, face, component feature, material, path, process specification, and surface texture, are all concepts represented in [2]. Welded joint, welding sequence, welding symbol, weld_element, weld definition and intermittent data are specific to the weld domain and are newly proposed. The AO Assembly_bond_definition, also found in [18], was determined to be an appropriate SUPERTYPE for the welding symbol. New AOs Welded_joint and Welding_definition are proposed as SUBTYPEs of Assembled_with_bonding and of Assembly_bond_definition, respectively. The relationship between Welded joint and Welding definition is essentially reversed from the default inherited from the SUPERTYPEs Assembly_joint and Assembled_with_bonding. That is necessary because one welding symbol can be applied to several joints. In the case that the component features are complex and more than one welding symbol is required, additional instances of Welded_joint will be provided by the pre-processor because each Welded_joint may only reference one instance of Welding_definition; in feature based design this is not a limitation as the end user defines the welding symbol by identifying 'arrow' and 'other' faces attached to the features and the pre-processor synthesizes Welded_joint as part of the mapping from the internal 
CAD model to the STEP model. Welding_definition is proposed as a name representing the properties in a welding symbol because this proposal does not include the graphic symbology. The AO Welding_symbol may be included (and bound to the Welding_definition) when the graphic symbology is added. There are five classes of welded joint: butt, corner, T, lap and edge that are modeled as SUBTYPEs of Welded_joint and five corresponding classes of Welding_definition.

A Welding_definition

- $\quad$ specifies the joint (or joints) it is helping to realize,

- $\quad$ includes a set of welds,

- includes designation of the arrow side,

- may include

- designation of the other side,

- $\quad$ supplemental information,

- $\quad$ surface texture,

- $\quad$ process specifications,

- a weld path,

- $\quad$ and auxiliary material.

There may be more than one welding operation in support of a welded joint and multiple welding operations occur in a sequence. Therefore a sequence relationship is included. The weld concept is represented by the AO Weld_definition. There are several types of welds supported, in accordance with [4] and [5]. They are identified as SUBTYPEs of Weld_definition in the ARM 
EXPRESS included herein. Three forms of intermittent data are supported: welds on one side only, welds located at same position on arrow and other side, welds that are placed first on one side then on the other (staggered). To support reporting inspection results, an explicit model of the weld elements derived from the intermittent data is provided, including unique identification. Parametric attributes are provided as required to satisfy [4] and [5]. In most cases, the terminology of [4] and [5] are consistent. In cases where consistency is not $100 \%$ [5] is used as the source for terms because there are more terms in [5]. The classification structure and separation of joint class, welding class, weld class and intermittent data classes make mapping from the source documents onto the Weld ARM straightforward. A weld context provides the ability to establish that the data being provided is in accordance with this AM. A separate context provides greater granularity for validation, verification and implementation. Consequently this AM can be considered to be an implementation level module in the STEP modular architecture. The Weld AM should be directly referenced by the implementation module ISO/TS 10303-442[15] upon publication.

Minor structural issues exist in the relationship between Assembled_with_bonding and Assembly_bond_definition. Because it appears that Assembly_bond_definition has never been implemented the corrections can be accomplished as part of stepmod maintenance. For development purposes, the schema interface provides explicit USE FROM and REFERENCE FROM to existing AMs. For development purposes, several AOs are explicitly included in the Weld AM that will be included in external AMs for publication. 


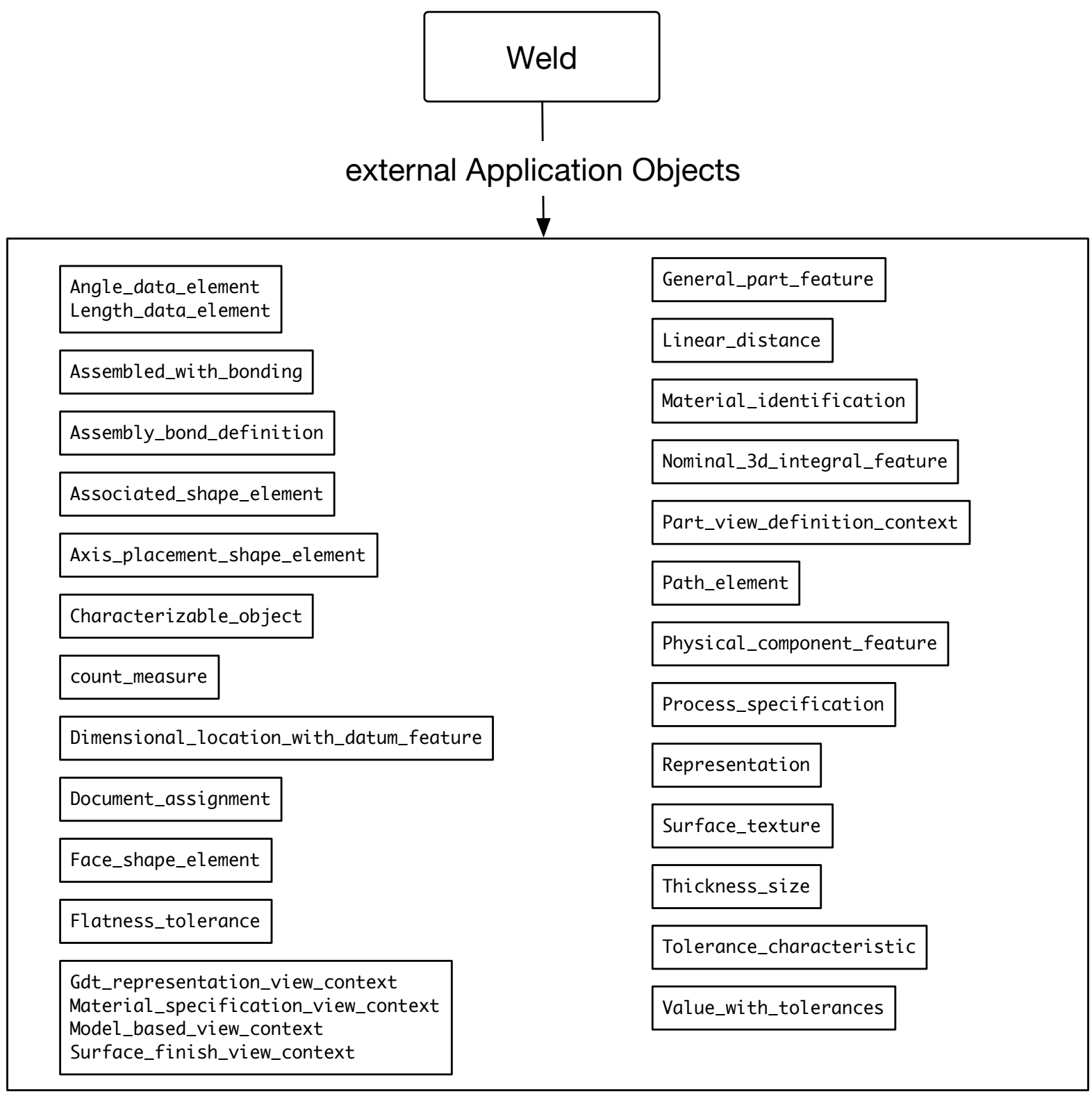

Figure 5 illustrates the external Application Objects directly referenced by the Weld application module ARM EXPRESS.

The Application Objects identified in Figure 5 are formally interfaced by the EXPRESS USE FROM declaration. They may define direct attributes or may be SUPERTYPEs of the Application Objects defined in the Weld ARM EXPRESS. The AOs also bound the scope of the externally referenced AMs as applied to the weld domain to facilitate validation and 
conformance processes in the weld domain.

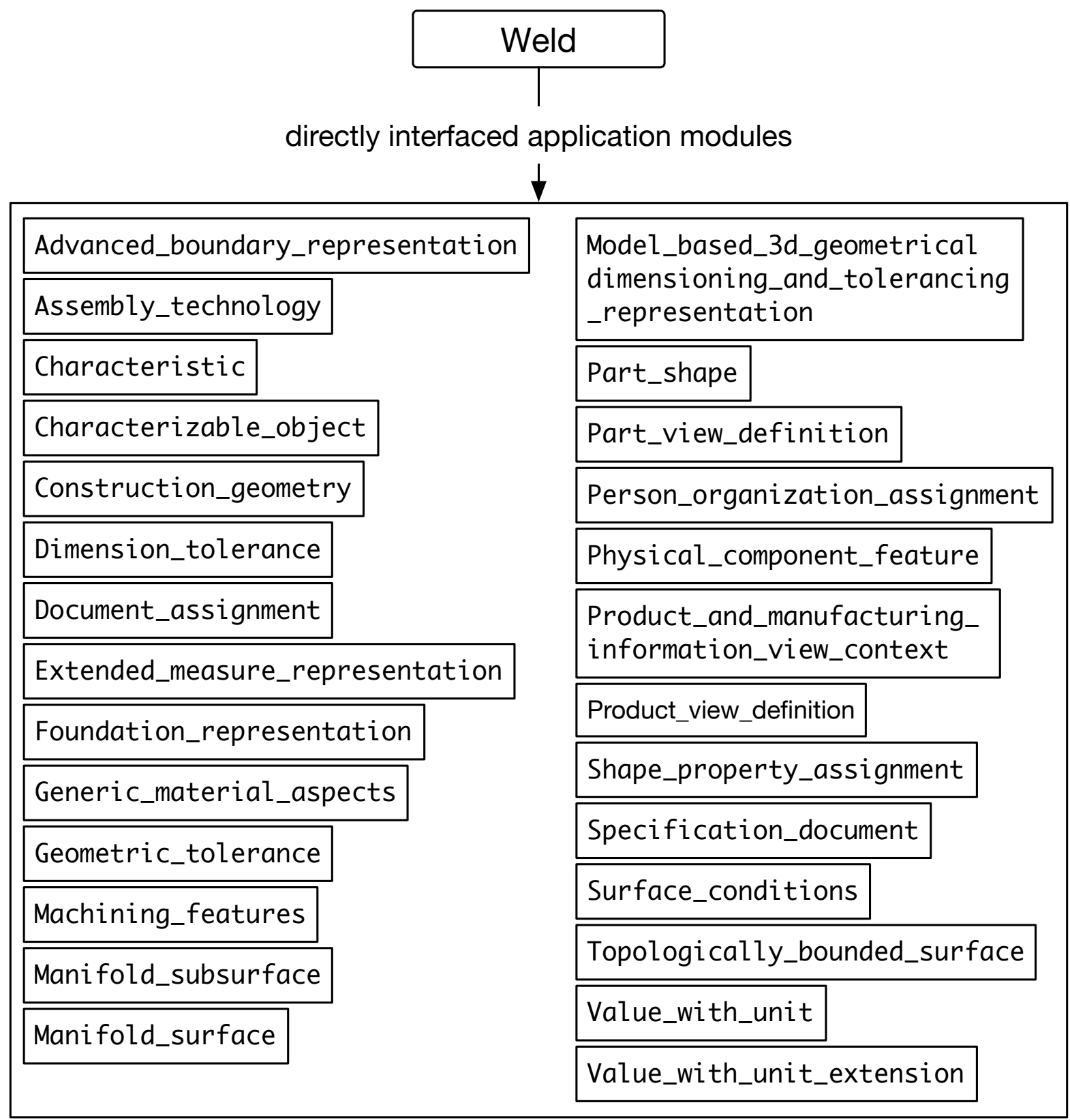

Figure 6 illustrates the external application modules that contain the directly referenced Application Objects included in Figure 5. 
The Application Modules illustrated in Figure 6 are formally interfaced by the EXPRESS USE FROM declaration. Several modules are interfaced because they include Application Objects that are provided to support geometric or dimensional tolerancing that may be included in the welding symbol but is not explicitly modeled. An example is AM ISO/TS 10303-1051 Geometric_tolerance(20) that includes Flatness_tolerance, and AM ISO/TS 10303-1050 Dimension_tolerance[21] that includes Thickness_size and Linear_distance. AM ISO/TS 10303-1816 Model based 3d geometrical dimensioning and tolerancing representation[22] was included to provide access to the correct context Application Objects (e.g., Gdt_representation_view_context) in a manner consistent with [2]. Several Application Modules in Table 6 are not in the scope of welding symbology. The consequence is that validation and testing will be more expensive due to the added effort of tracking out of scope items. The out of scope modules should be removed from the interfaces during the next phase of the development process as identified in[11] by moving the Application Objects interfaced into a foundation module[11] that does not have a narrow domain scope. An example is AM Machining_features[19] that includes Face_shape_element. Face_shape_element and Face_shape_element_relationship should be moved to AM ISO/TS 10303-1032 Shape_property_assignment[23]. Another example is AM Geometric_tolerance[20] that includes Plane_shape_element and Point_shape_element. Those AOs should also be moved to [23]. 


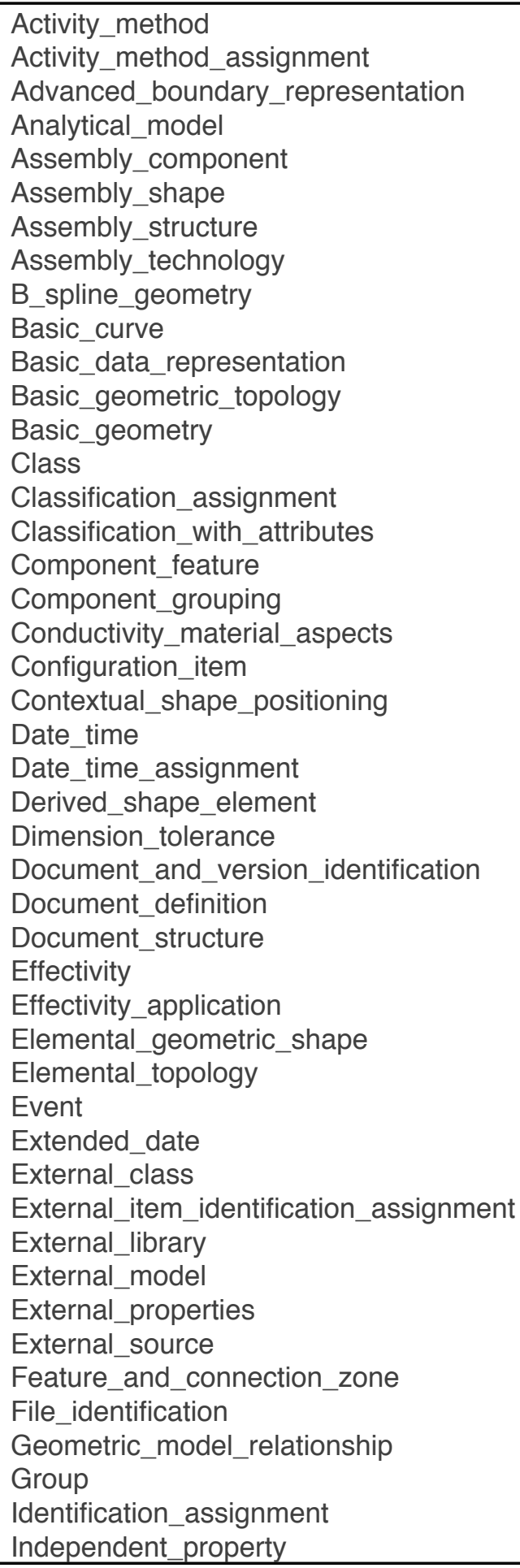

Table Two identifies the Application Modules indirectly included in Weld module by executing a short to long form transform on the ARM EXPRESS as defined in [10]. 


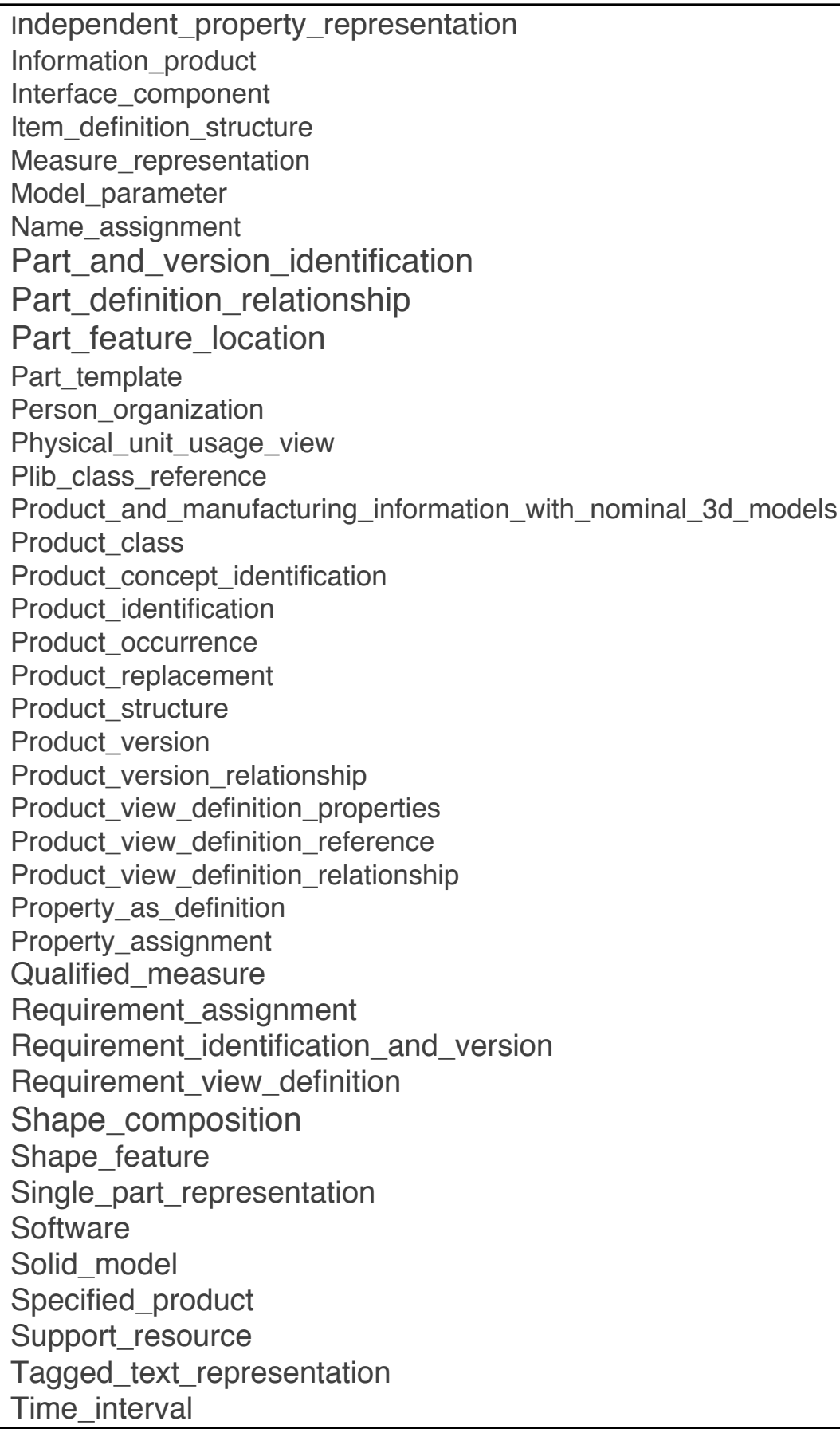

Table Two continued.

Several Application Modules in Table Two are not in the scope of welding symbology.

The consequence is that validation and testing will be more expensive due to the added effort of tracking out of scope items. The out of scope modules should be removed from the scope during 
the next phase of the development process as identified in[11]. Examples include Tagged_text_representation[24], and Information_product[25]. 


\section{Information model maintenance}

\section{Surface texture}

Surface_texture_parameters.parameter_name and

Surface_texture_parameters.parameter_value are incorrectly described in ISO/TS

10303-1110:2014-02 Surface conditions AM[26]. Those descriptions will be corrected in the next publication of ISO/TS 10303-1110 Surface conditions AM as part of the STEPmod maintenance task of AP 242 ed2 development.

\section{Thread}

Several STEPmod maintenance items relate to the existing Thread AO in [19].

Issue 4236 identifies needed clarifications on the attributes Thread.thread_hand and Thread.inner_or_outer_thread in the ARM descriptions and EXPRESS. Those clarifications were incorporated in the Screw_thread module proposed in this work and can be incorporated into [19] as part of a maintenance activity when the manufacturing APs are migrated to STEPmod.

Issue 4237 relates to the Thread.applied_shape attribute that specifies the base shape of the part from which machining processes remove material to result in the shape of the thread. The model used for Thread.applied_shape to establish that relationship to the base shape is shared amongst ISO 10303-224[27] (AP 224) up through edition 3, ISO 10303-238[28] (AP 238) and ISO 14649-10[29]. Those APs are manufacturing context specific. ISO 10303-214[30] edition 3 (AP 214) has a different approach in the ARM but the mapping results in the same formal interface. There is however a distinct difference between a design context and a manufacturing context. The modeling approach of the manufacturing APs is that there is a 
volume of material from which material is removed to generate a net shape, while the design context approach is feature based and does not acknowledge a particular process manufacturing may use to realize the feature. The issue of design features as compared to manufacturing features is addressed in[31].

Issue 4783 includes mapping errors in[19]. The detailed recommendations for issue 4783 are provided in[32]. The corrections can be incorporated as part of a maintenance activity when the manufacturing APs are migrated to STEPmod.

Issue 5116 includes a discussion of the relationship between Thread and Partial_area_definition and has lead to the proposal for a new Screw_thread module for the design context. Clarification of the relationship between Thread and Partial_area_definition should be deferred until the manufacturing APs are migrated to STEPmod. 
Updated architecture of PMI in STEP

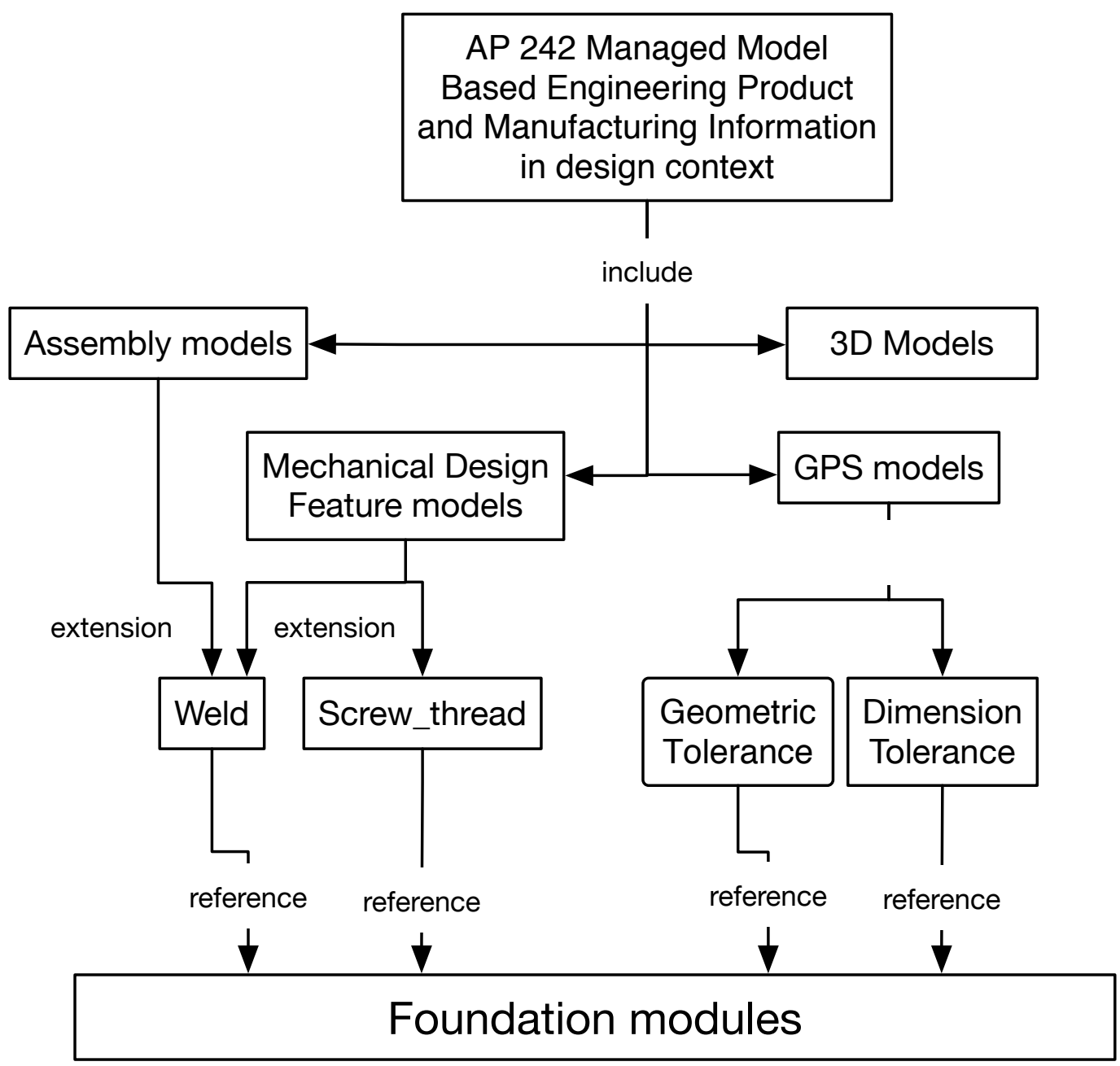

Figure 7 illustrates the updated abstract document architecture proposed to be included in edition two of[33]

The models related to the addition of domain of screw threads and weld in the second edition of [2] can be gathered under the Product and Manufacturing Information (PMI) concept. Specifically for screw thread and weld we are concerned only with a design context. The AP will support PMI with 3 dimensional geometric models, assembly models, mechanical design feature models, and Geometric Product Specification (GPS) models. Screw thread data are independent 
of the model geometric representation. Welding data require the equivalent of a geometric face in the geometric model. Explicit product_definition_context[34] entities are provided in the PMI design context to support more efficient interface development. The weld domain requires assembly models that include part occurrence as a component, component features and assembly joint. Representation of those concepts are available in[2]. The mechanical feature models in[2]that include e.g., Definitional_shape_element[35] are extended by the inclusion of Weld and Screw_thread. Existing Geometric Product Specification (GPS) models for Dimension tolerance and for Geometric tolerance in[2] are re-used, as are lower level shape and shape property models included in the Foundation modules in Figure 7. Screw thread and weld data relies on the identification capability provided in the existing mechanical design feature models, e.g., unique constraints on shape_aspect.id[34] and on geometric_item_specific_usage[23] to link production measured data back to design features. Weld extends those identification data to include intermittent weld identification.

\section{Upward compatibility and deprecation}

Because Screw thread and Weld are new capabilities in the design context, there are no upward compatibility issues determined to exist with Screw thread and Weld. 


\section{Screw thread ARM EXPRESS}

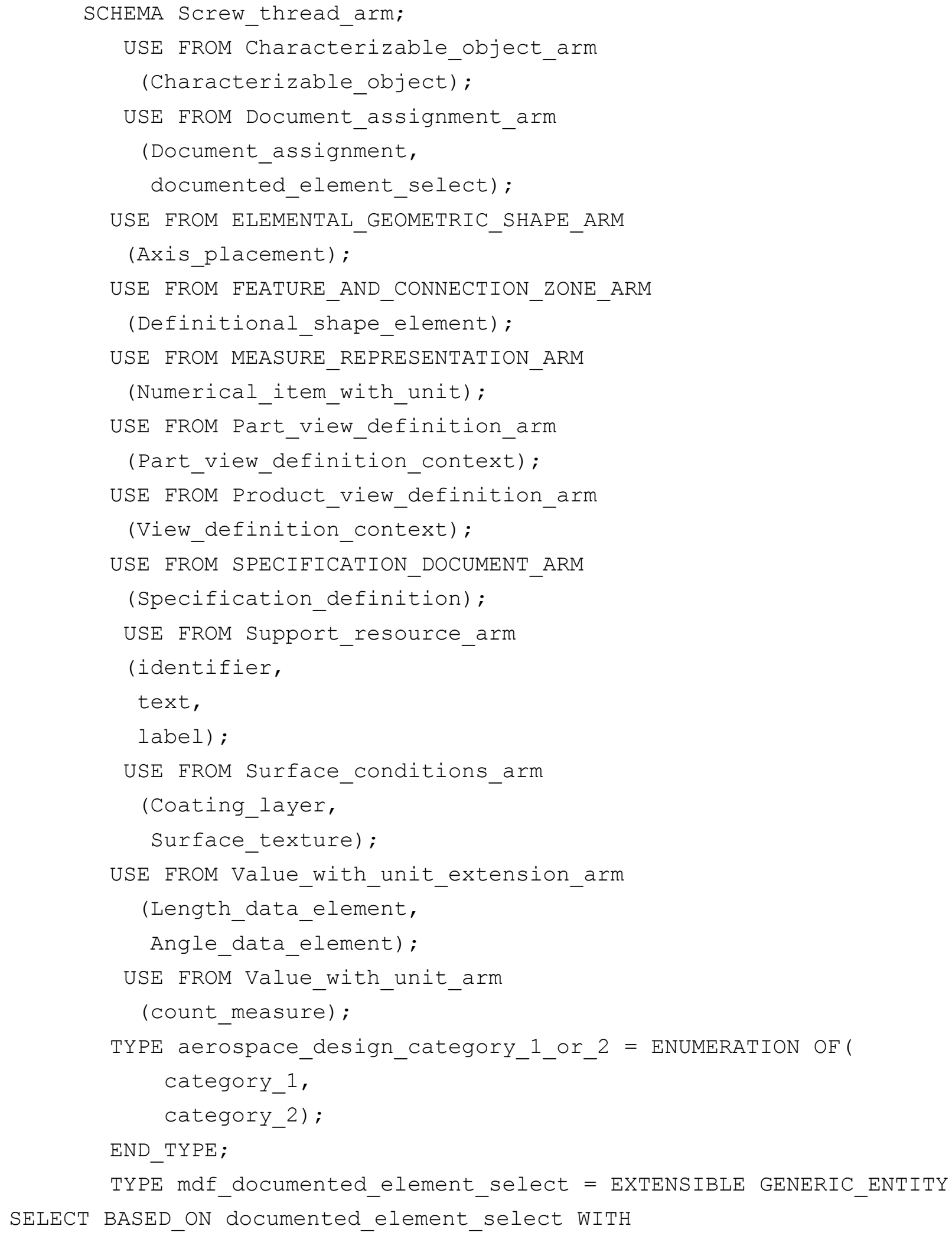




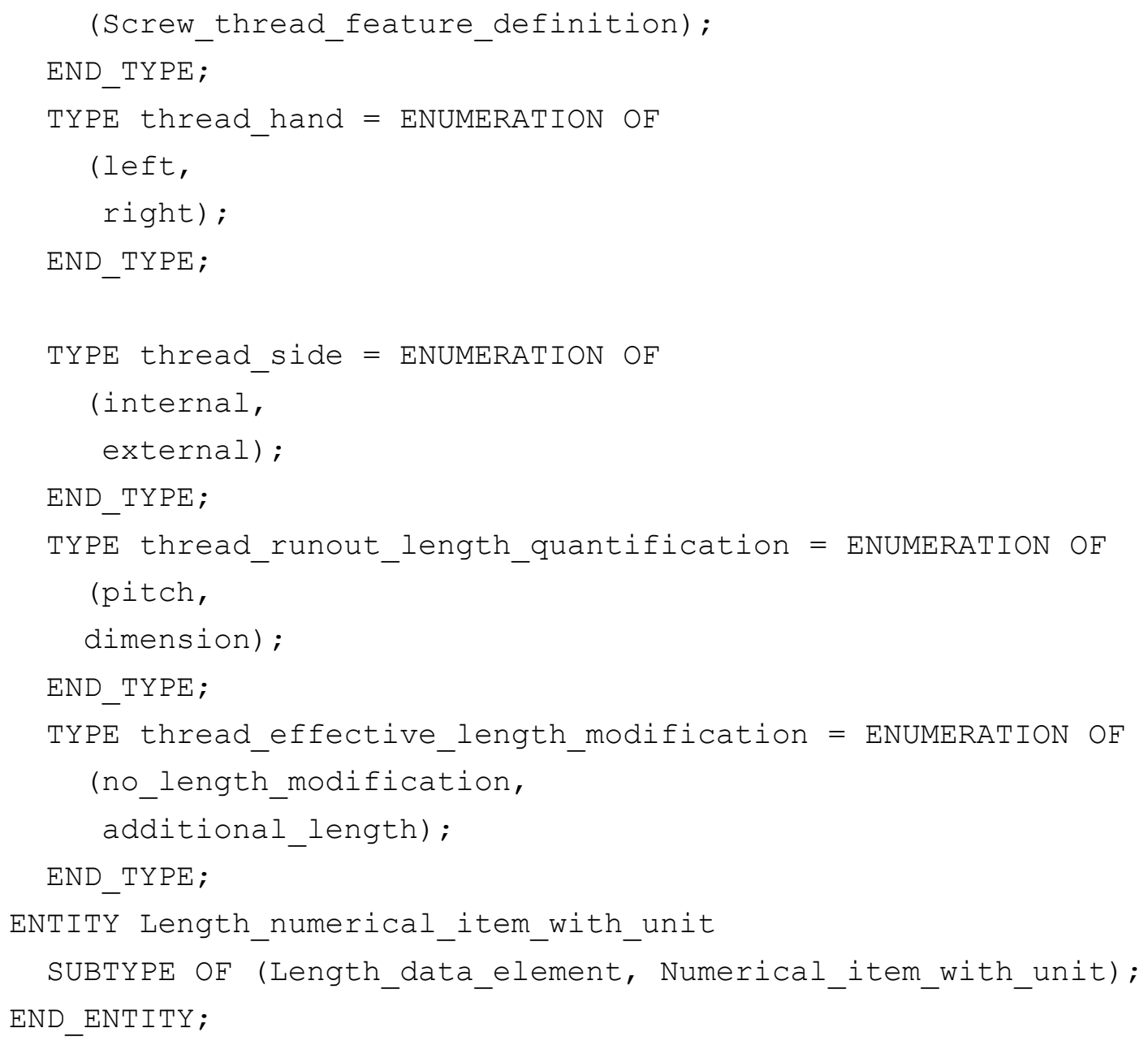

- This AO provides the ability to declare that a data element that may have tolerances supplied as part of the data element is a length. When integrated into STEPmod, Length_numerical_item_with_unit will be included in ISO/TS 10303-1753 AM Value_with_unit_extension[18].

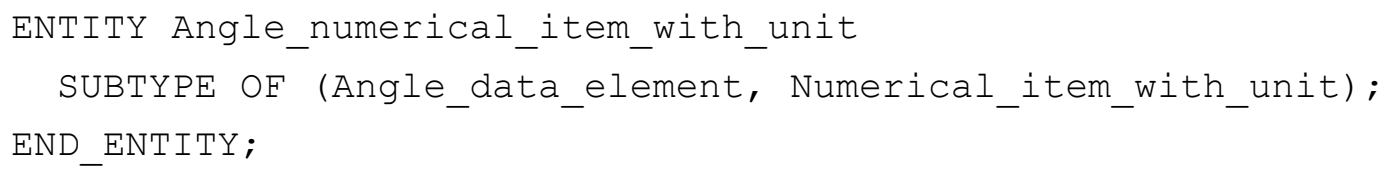

- This AO provides the ability to declare that a data element that may have tolerances supplied as part of the data element is angular. When integrated into STEPmod, Angle_numerical_item_with_unit will be included in [18].

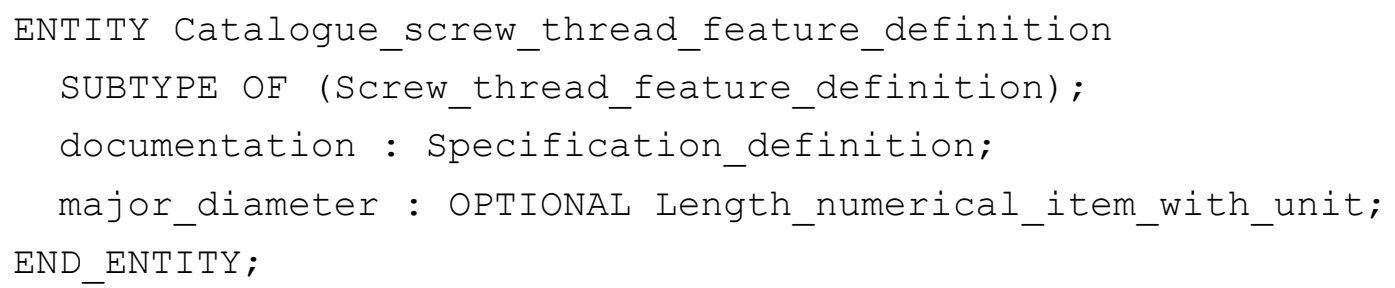


—Catalogue_screw_thread_feature_definition is derived from AO Catalogue_thread in [19] but does not include the base shape construct. The major_diameter is constrained to be a length.

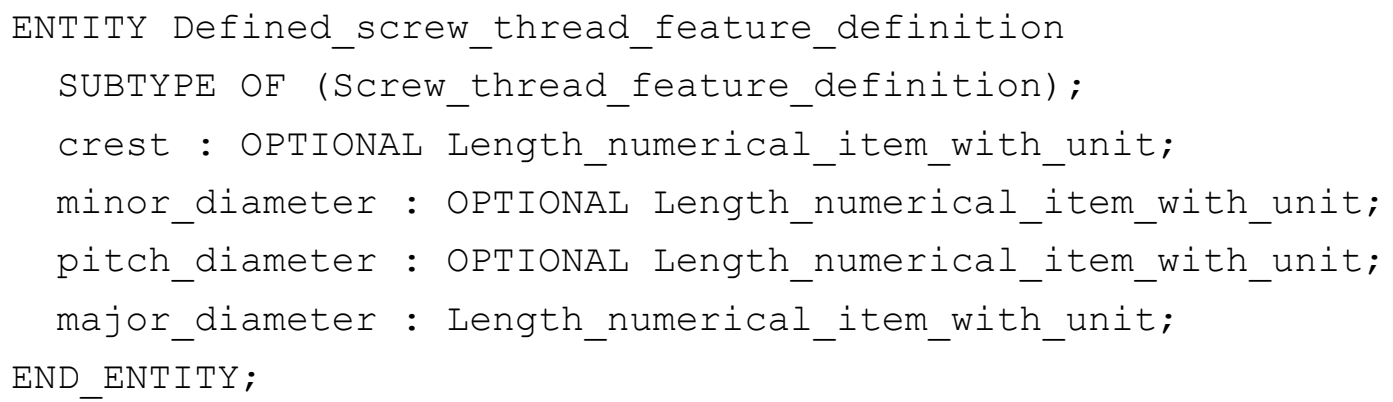

— Defined_screw_thread_feature_definition is derived from AO Defined_thread in [19] but does not include the base shape construct. The attributes are constrained to be lengths.

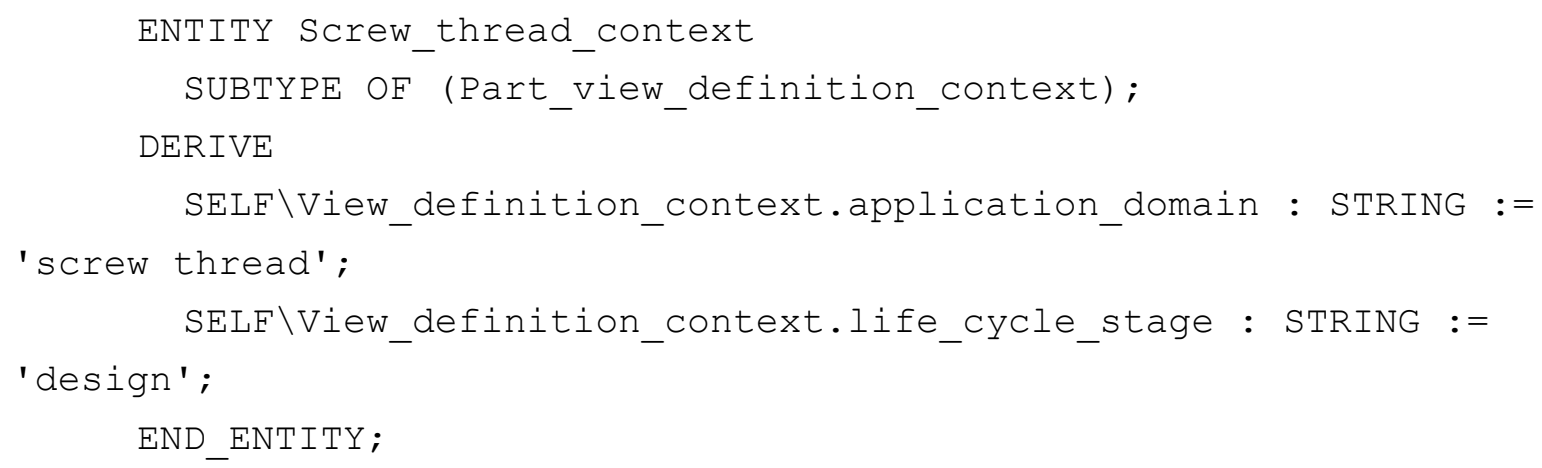

- Screw_thread_context provides the ability to establish that the data being provided is in accordance with this AM. A separate context provides greater granularity for validation, verification and implementation.

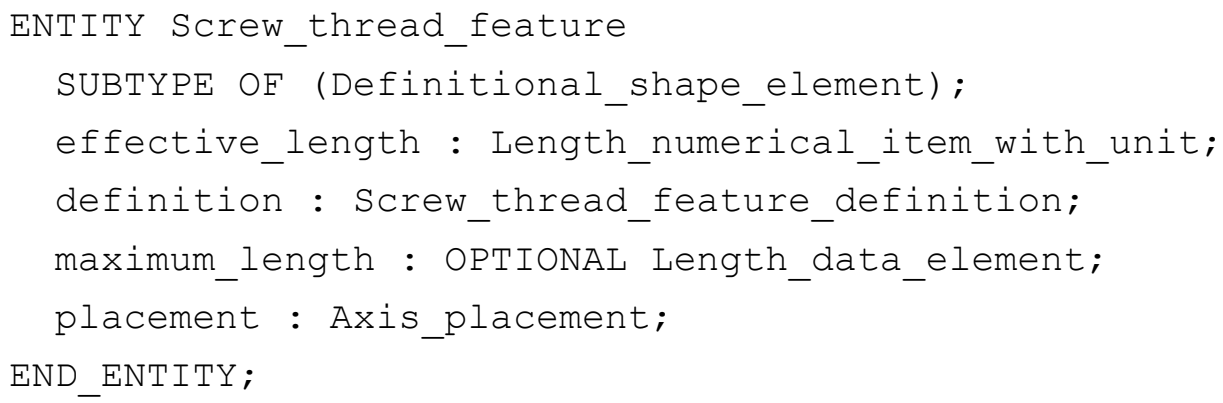

— Screw_thread_feature is derived from AO Partial_area_definition in [19] but does not include the base shape construct and is a Definitional_shape_element providing straightforward integration into other aspects of the design model. Specifically the critical attribute Screw_thread_feature.definition will be interpreted to be a shape_aspect_relationship in ISO 10303-41[34]. The Screw_thread_feature.effective_length and Screw_thread_feature.maximum_length attributes are constrained to be length AO types to ease 
mapping specification and MIM development.

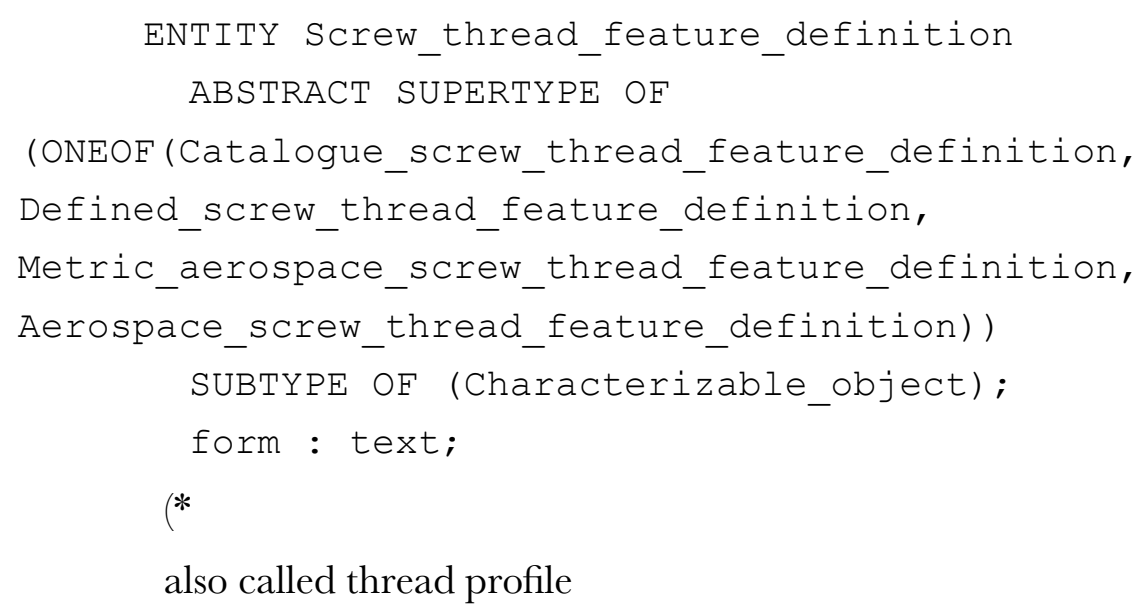

The cross-sectional shape of a thread is often called its form or threadform. It may be square, triangular, trapezoidal, or other shapes. The terms form and threadform sometimes refer to all design aspects taken together (cross-sectional shape, pitch, and diameters).

The form attribute provides the same data as Thread.form in [19].

*) gaging_system : OPTIONAL text;

- The gaging_system attribute provides the value for gaging specified in [7] or [8]. hand : thread_hand;

- The hand attribute provides the same data as Thread.form in [19]. side : thread_side;

- The side attribute provides the same data as Thread.side in [19]. runout : OPTIONAL Thread_runout;

- The runout attribute provides similar data as Thread.runout in [19]. series : OPTIONAL text;--coarse, fine,..

(*

series of threads, i.e. groups of diameter and number of threads per inch combinations distinguished from each other by the number of threads per inch associated with any given thread diameter.

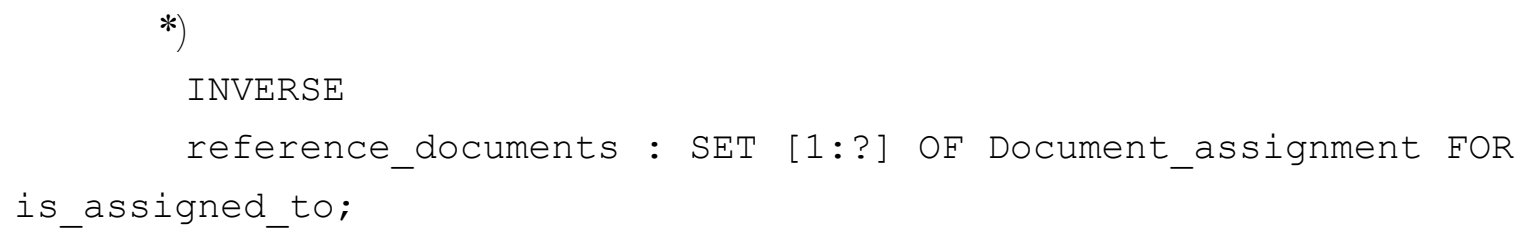

- Specifies the document that controls the interpretation of the data provided. END_ENTITY; 
- Screw_thread_feature_definition is derived from the AO Thread in [19] but does not include the base shape construct and is a Characterizable_object providing straightforward integration into other aspects of the design model. The attributes that are representations of length concepts are constrained to be length AO types.

ENTITY Metric_aerospace_screw_thread_feature_definition

SUBTYPE OF (Screw_thread_feature_definition);

tolerance_class : text;

- Tolerance class is specified in [8] nominal_size : count_measure;

—Nominal_size is nominal diameter in millimetres as specified in [8]. pitch : count measure;

- Pitch is the distance between threads in millimetres as specified in [8]. special_thread : BOOLEAN;

-A special thread is defined in [8].

END_ENTITY;

- A Metric_aerospace_screw_thread_feature_definition is a minimal set of parametric data needed to specify commonly used metric screw threads as defined in [8].

ENTITY Aerospace_screw_thread_feature_definition

SUBTYPE OF (Screw_thread_feature_definition);

coating : OPTIONAL Coating_layer;

-Aerospace threads may have coating applied.

fit_class : text;

— The fit_class attribute provides the same data as Thread.fit_class in [19] except that it does not include the metric case.

design_category : OPTIONAL aerospace_design_category_1_or_2;

-Design category is defined in [7].

$(*$

not in iso 3161 ?

*)

major_diameter : OPTIONAL Length_numerical_item_with_unit;

-Diameters are nominal unless special_thread is indicated. See [7] or [8]. minor_diameter : OPTIONAL Length_numerical_item_with_unit;

-Diameters are nominal unless special_thread is indicated. See [7] or [8]. number_of_threads_per_inch : OPTIONAL count_measure; pitch_diameter : OPTIONAL Length_numerical_item_with_unit; 
—Diameters are nominal unless special_thread is indicated. See [7] or [8]. qualifying_information : OPTIONAL text;

(*

Occasionally it is necessary to modify the major diameter of external threads or the minor diameter of internal threads in order to fit a specific purpose, but without changing the pitch diameter limits (it should be noted that existing gauges may be used to accept such threads). Such threads shall be specified with the established thread designation followed by the modified crest diameter limits and the designation "MOD".

In that case "MOD" would be the qualifying_information value.

*) root_radius : OPTIONAL Length_numerical_item_with_unit;

- Root_radius is not toleranced unless special_thread is indicated as noted in [7] or [8]. Screw_thread_feature_definition\series : text;

(*

graded pitch: coarse, fine, extra fine constant pitch: 8, 12, 16 threads per inch

$*)$

- The value of series is for example: 'graded pitch, coarse'.

- The value of series is for example: 'constant pitch, 8 threads per inch'. significant_digits : OPTIONAL INTEGER;

- Significant digits is included when necessary, in accordance with [7] or [8]. special_thread : BOOLEAN;

-A special thread as defined in [7].

-A special thread will usually provide qualifying information as identified in the attribute 'qualifying_information'.

surface_roughness : OPTIONAL surface_texture;

- Surface roughness may be provided.

DERIVE

pitch : REAL := 1.0/number_of_threads_per_inch;

END_ENTITY;

-The Aerospace_screw_thread_feature_definition is based on [7] and [14].

ENTITY Thread_runout;

length_of_runout : Length_numerical_item_with_unit;

length_quantification : thread_runout_length_quantification; 
effective_length_modification :
thread_effective_length_modification;

END_ENTITY;

- Thread_runout is derived from Thread_runout in [19] but provides a clarified model

for length_quantification and for effective_length_modification, as well as constraining

length_of_runout to be a length AO type.

END_SCHEMA; 


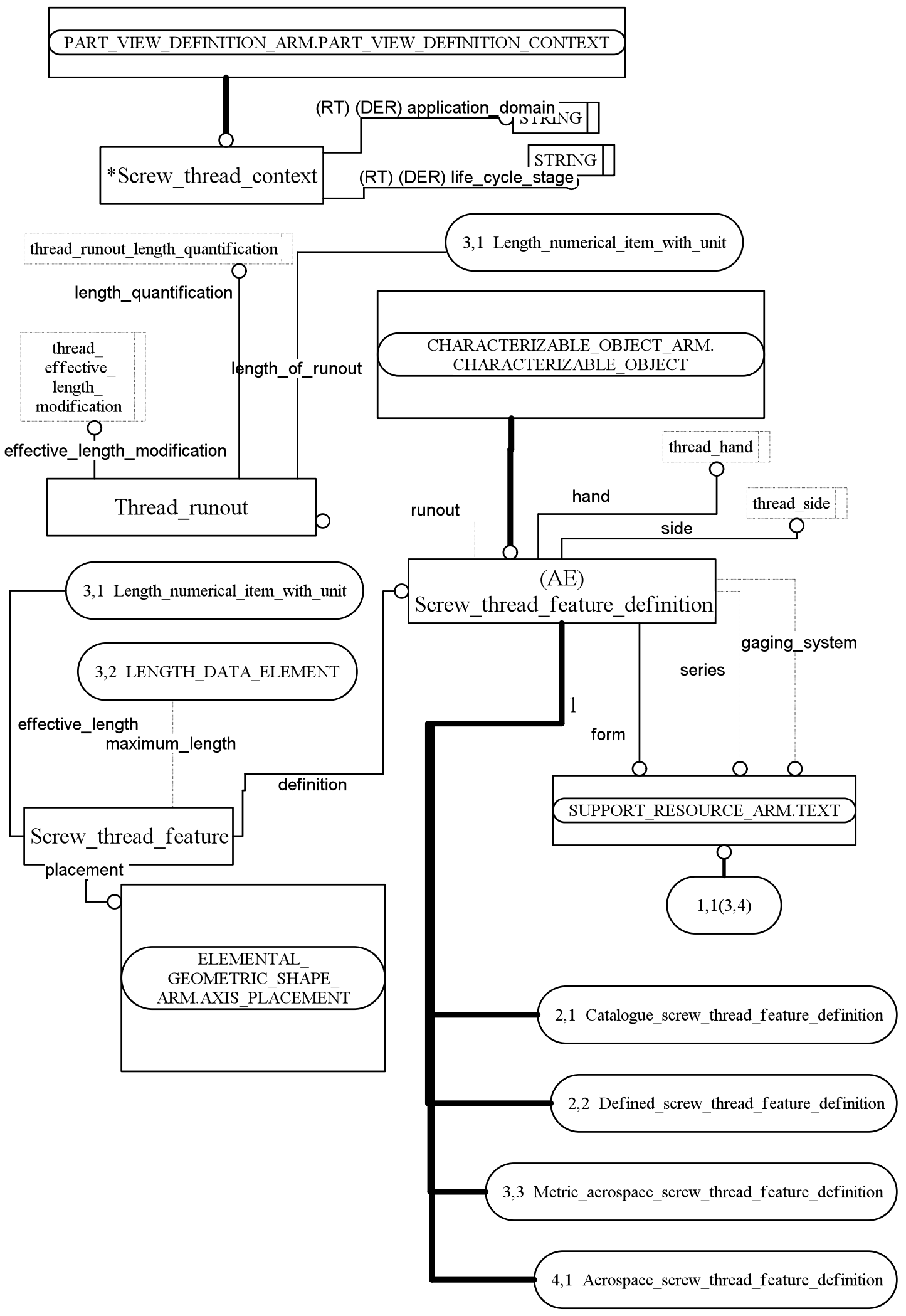

Figure 8 Screw thread ARM Diagram 1 of 4

On Extending an ISO Standard for Exchanging Product Manufacturing Information 


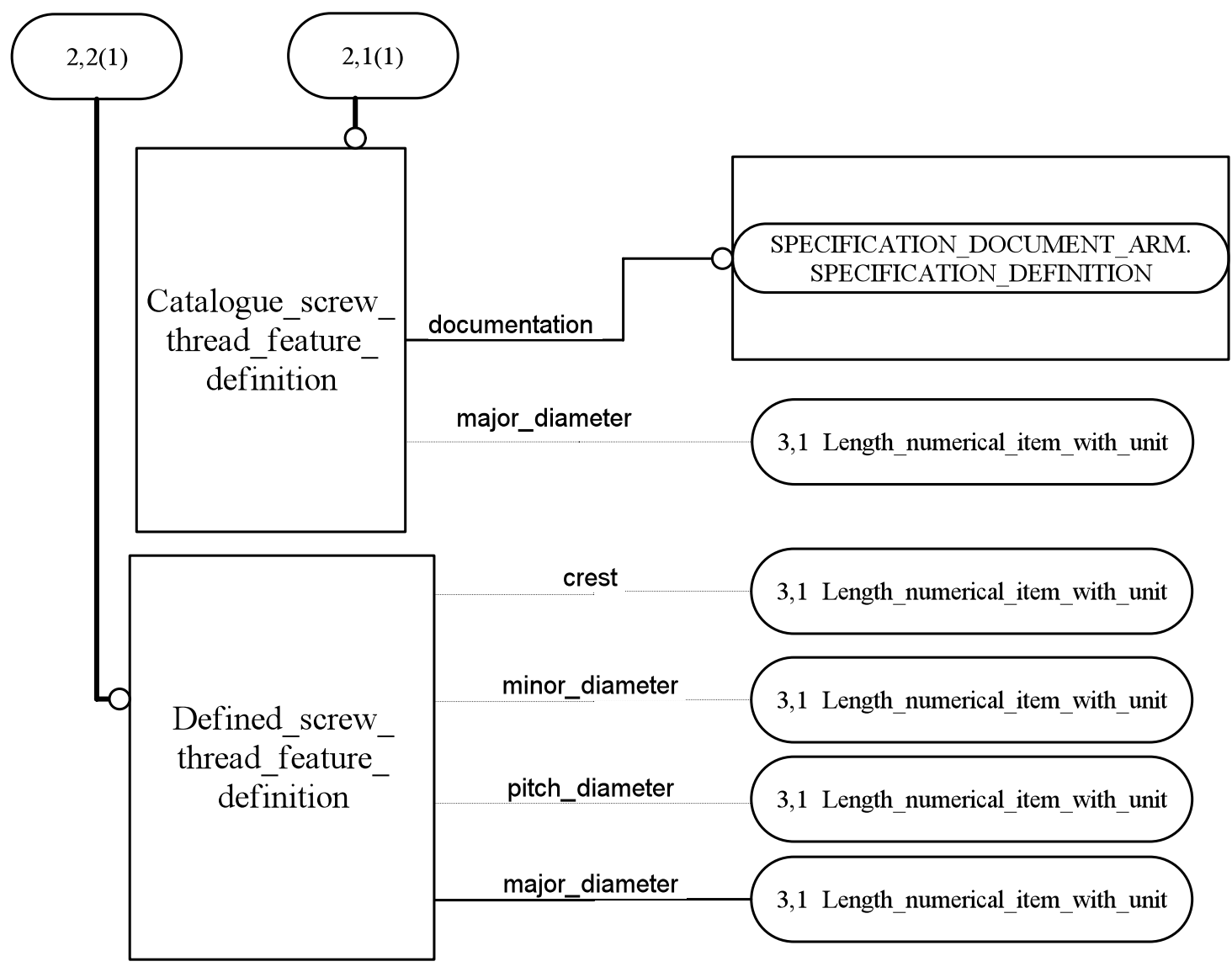

Figure 9 Screw thread ARM Diagram 2 of 4 

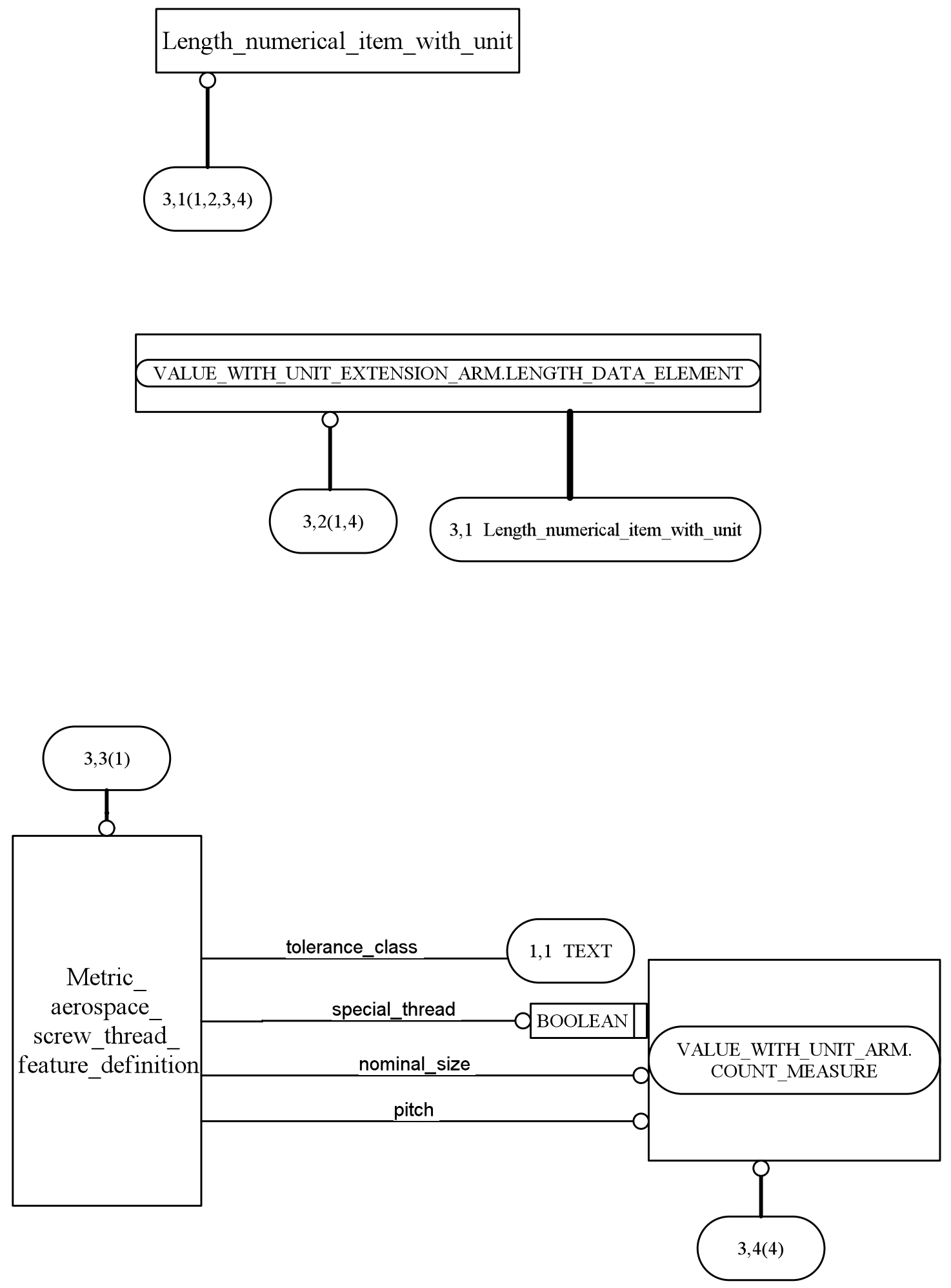

Figure 10 Screw thread ARM Diagram 3 of 4 


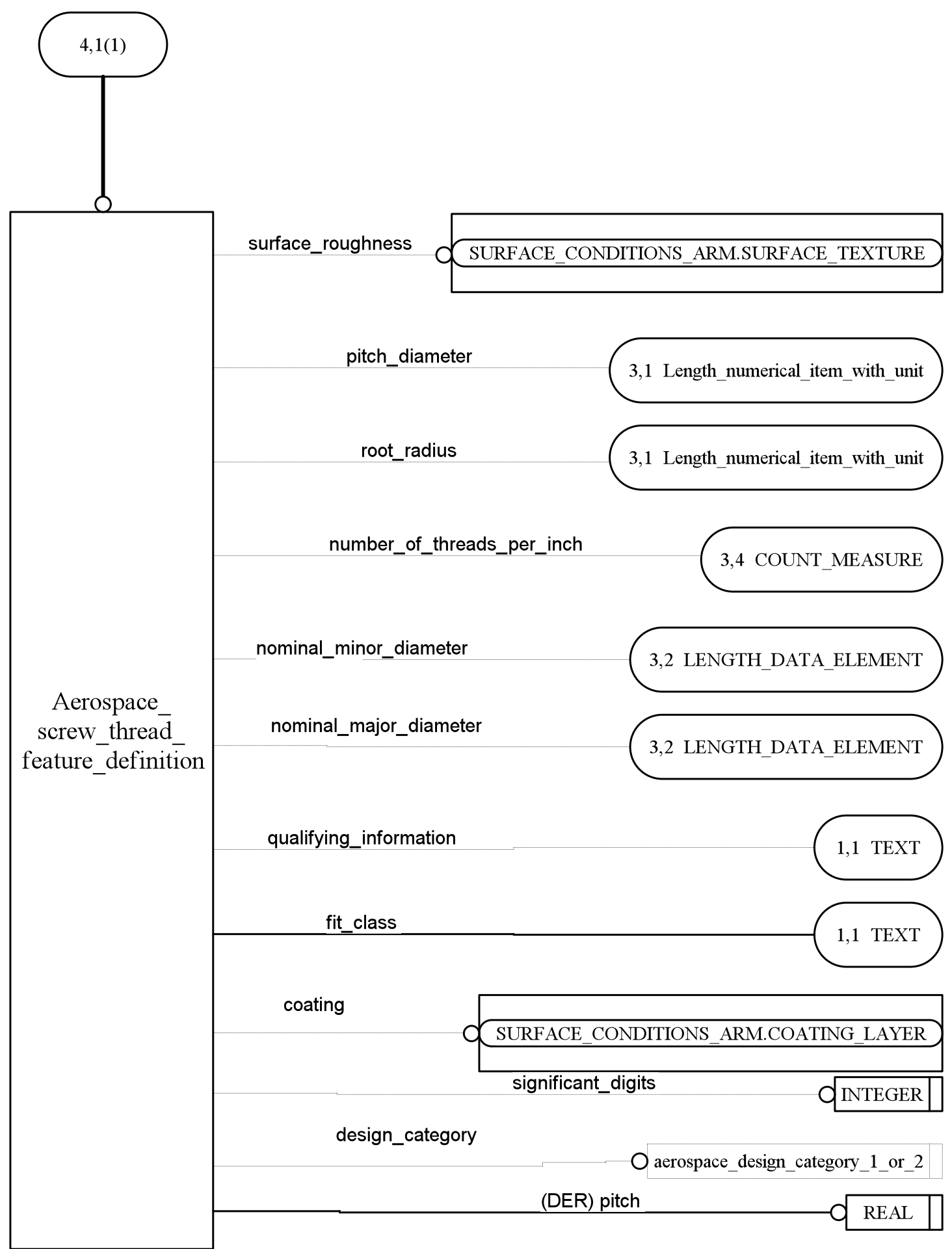

Figure 11 Screw thread ARM Diagram 4 of 4 


\section{Weld ARM EXPRESS}

SCHEMA Weld_arm;

USE FROM Advanced_boundary_representation_arm; -- ISO/TS $10303-1514$

USE FROM Assembly_technology_arm

(Assembled_with_bonding, Assembly_bond_definition);

USE FROM Characteristic_arm

(Length_tolerance_characteristic, Maximum_tolerance_characteristic, Minimum_tolerance_characteristic, Nominal_tolerance_characteristic, Plus_minus_tolerance_characteristic, Qualified_tolerance_characteristic, Statistical_tolerance_characteristic, Symmetrical_tolerance_characteristic, Tolerance_characteristic, Typical_tolerance_characteristic);

USE FROM Characterizable_object_arm (Characterizable_object);

USE FROM Construction_geometry_arm; -- ISO/TS 10303-1131

USE FROM Dimension_tolerance_arm

(Thickness_size,

Linear_distance);

USE FROM Extended_measure_representation_arm (Value_with_tolerances);

USE FROM Foundation_representation_arm

(Representation); USE FROM Generic_material_aspects_arm

(Material_identification);

USE FROM Geometric_tolerance_arm

(Axis_placement_shape_element, Dimensional_location_with_datum_feature, Flatness_tolerance); USE FROM Machining_features_arm 


$$
\begin{aligned}
& \text { (Face_shape_element, } \\
& \text { Path_element); }
\end{aligned}
$$

- These two AOs should be moved to another AM to avoid USE FROM

\section{Machining_features_arm}

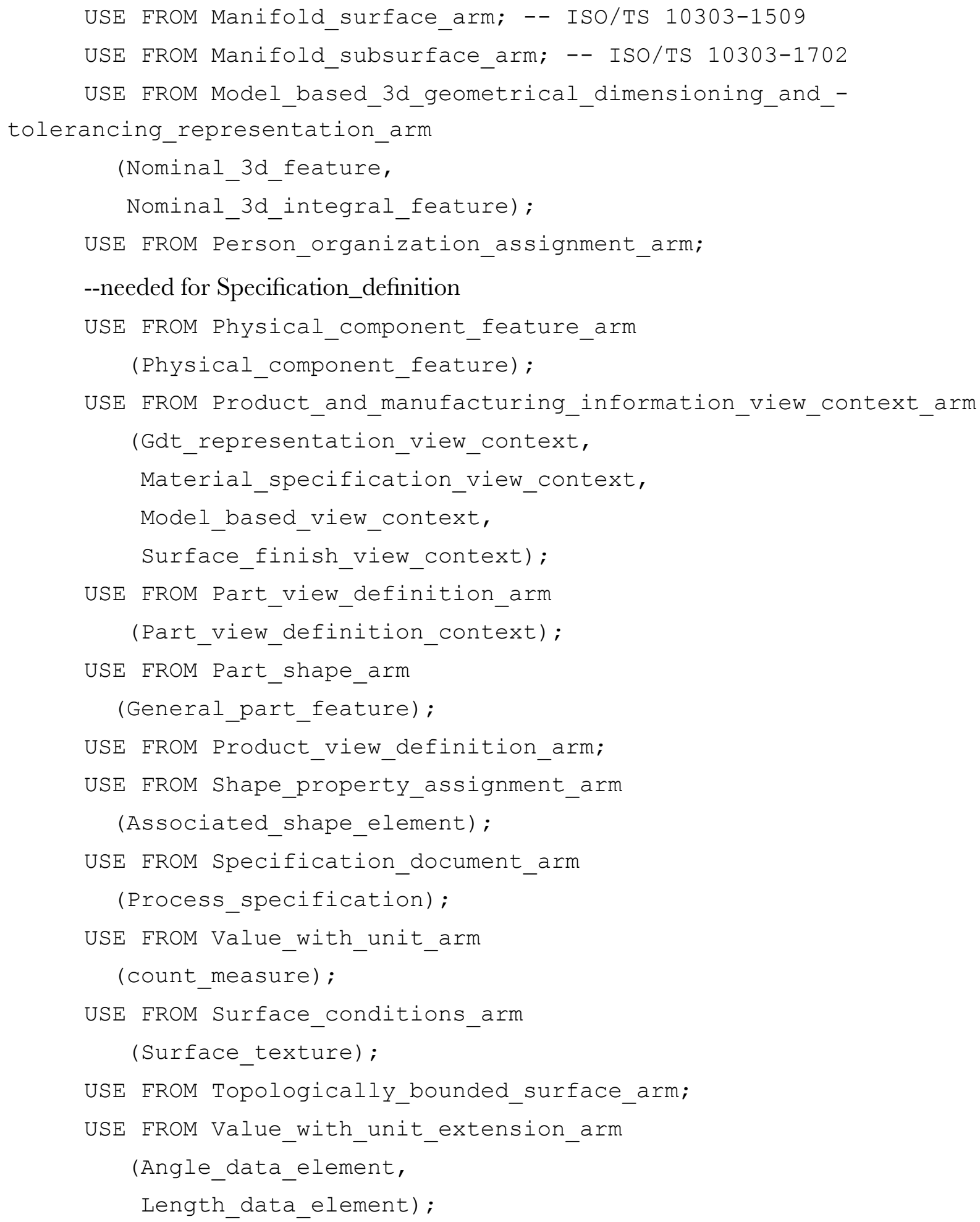




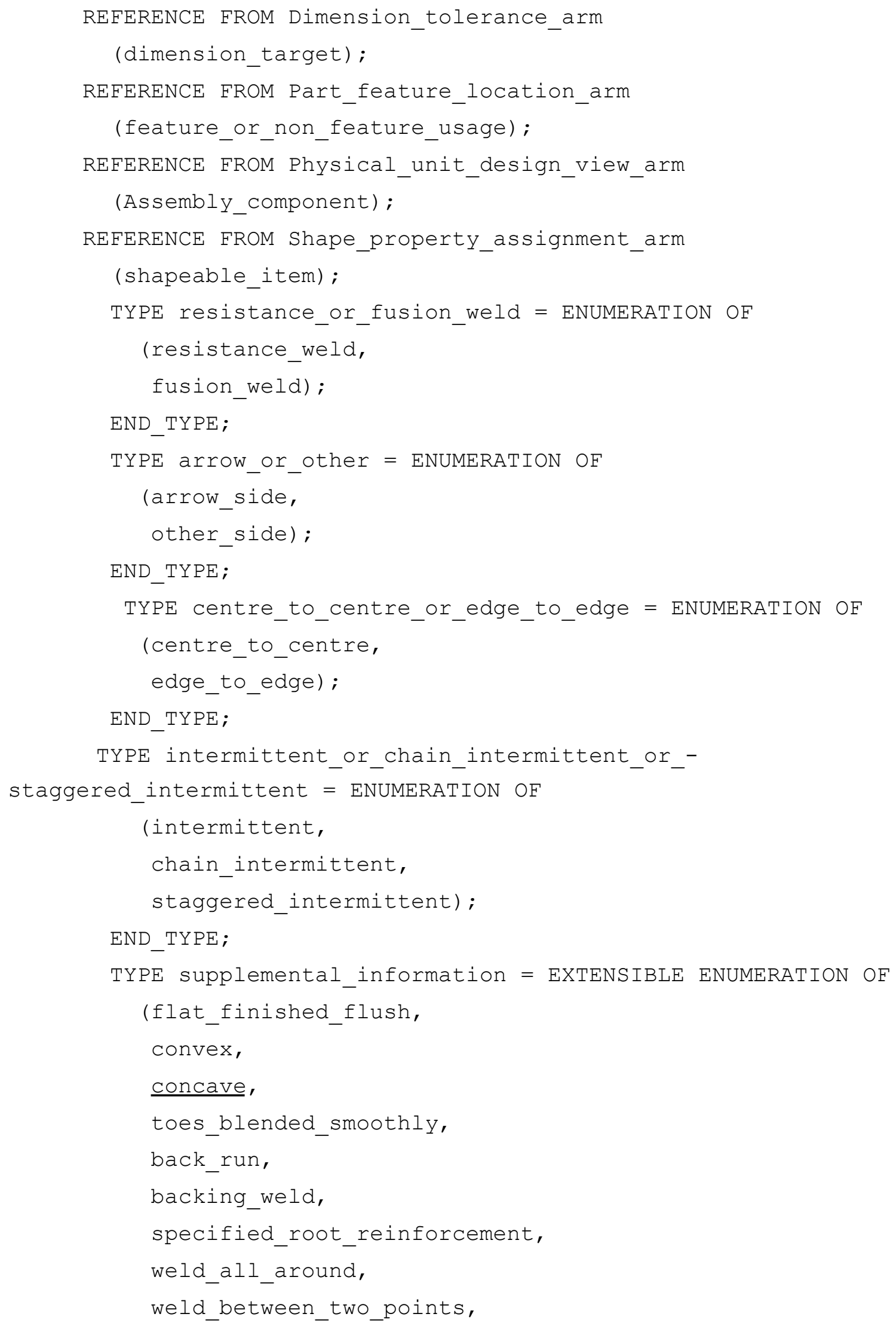




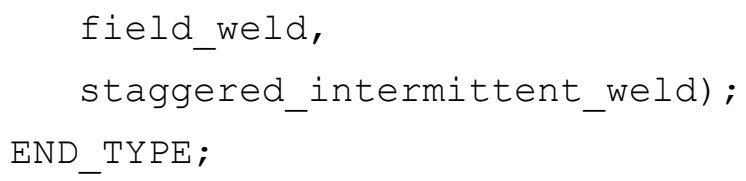

- In [5] this information is referred to as supplementary symbols.

TYPE weld_auxiliary_material_role = ENUMERATION OF

(consumable_insert,

permanent_backing,

removable_backing,

spacer,

unspecified_backing);

END_TYPE;

- In [5] auxiliary material is included in supplementary symbols.

TYPE weld_dimension_target $=$ SELECT BASED_ON dimension_target

WITH (Nominal_3d_feature);

END_TYPE;

- A target is needed for the dimension tolerance associated with a flatness tolerance that may be applied to the finished workpiece.

TYPE weld_shapeable_item = SELECT BASED_ON shapeable_item WITH

(Characterizable_object);

END_TYPE;

-Weld_shapeable_item is needed for STEPmod integration.

ENTITY Angle_tolerance_characteristic

SUBTYPE OF(Tolerance_characteristic);

WHERE

WR1 : ('CHARACTERISTIC_ARM.-

STATISTICAL_TOLERANCE_CHARACTERISTIC' IN TYPEOF(SELF)) OR

('CHARACTERISTIC_ARM.SYMMETRICAL_TOLERANCE_CHARACTERISTIC' IN

TYPEOF (SELF)) OR

( 'CHARACTERISTIC_ARM.PLUS_MINUS_TOLERANCE_CHARACTERISTIC'

IN TYPEOF (SELF)) OR

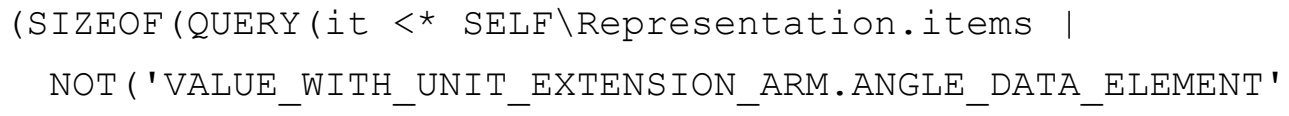

IN TYPEOF(it))

\author{
)) = 0); \\ WR2 : NOT ('CHARACTERISTIC_ARM. ' + \\ 'SYMMETRICAL_TOLERANCE_CHARACTERISTIC' IN TYPEOF(SELF)) OR
}

On Extending an ISO Standard for Exchanging Product Manufacturing Information 
(SIZEOF (QUERY (it <* SELF $\backslash$ Representation.items । ('VALUE_WITH_UNIT_EXTENSION_ARM.ANGLE_DATA_ELEMENT' IN

TYPEOF (it))

$$
\text { )) (1); }
$$

WR3 :

NOT ('CHARACTERISTIC_ARM.STATISTICAL_TOLERANCE_CHARACTERISTIC' IN TYPEOF (SELF)) OR

(SIZEOF (QUERY (it <* SELF\Representation.items ।

('VALUE_WITH_UNIT_EXTENSION_ARM.ANGLE_DATA_ELEMENT' IN

TYPEOF (it))

$$
\text { )) = 1); }
$$

WR4 : NOT EXISTS(SELF $\backslash$ Representation.description); WR5 :

NOT ('CHARACTERISTIC_ARM.PLUS_MINUS_TOLERANCE_CHARACTERISTIC' IN TYPEOF (SELF)) OR

$$
\text { (SIZEOF (QUERY (it <* SELF \Representation.items । }
$$

NOT( ('EXTENDED_MEASURE_REPRESENTATION_ARM.VALUE_WITH_TOLERANCES' IN TYPEOF(it)) AND

('VALUE_WITH_UNIT_EXTENSION_ARM.ANGLE_DATA_ELEMENT'

IN TYPEOF (it Value_with_tolerances.item_value)))

)) = 0);

END_ENTITY;

- Angles may have tolerance associated. This AO will be added to [18].

ENTITY Butt_joint

SUPERTYPE OF (ONEOF (Flanged_Butt_joint,

Inclined_Butt_joint))

SUBTYPE OF (Welded_joint);

SELF \assembled_with_bonding.default_bond_definition :

Butt_joint_welding_definition;

END_ENTITY;

—Butt_joint specifies a butt joint specific welding definition.

ENTITY Butt_joint_welding_definition

SUBTYPE OF (Welding_definition);

SELF\Welding_definition.welds : set [1:?] OF

Butt_weld_definition;

INVERSE 


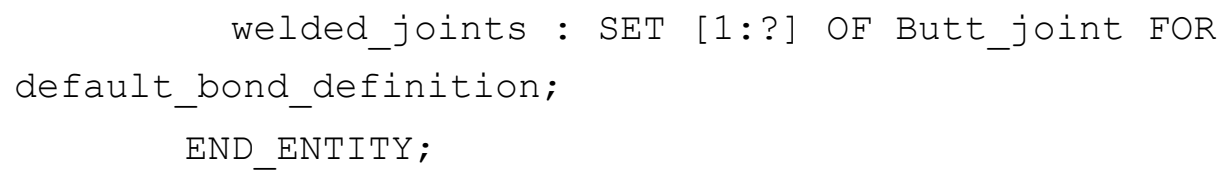

—Butt_joint_welding_definition specifies a set of Butt_weld_definitions and associates those weld definitions to one or more Butt_joints.

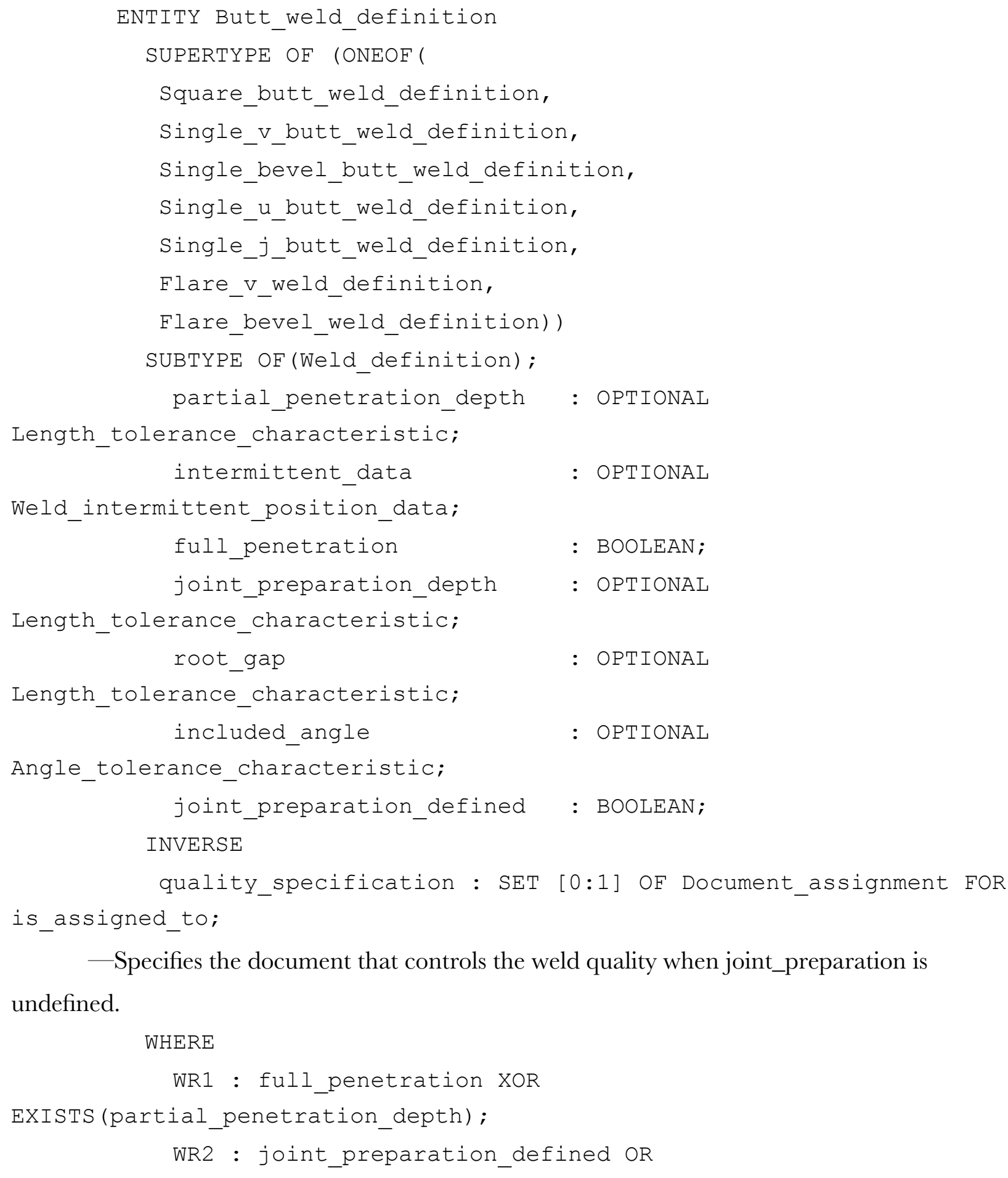




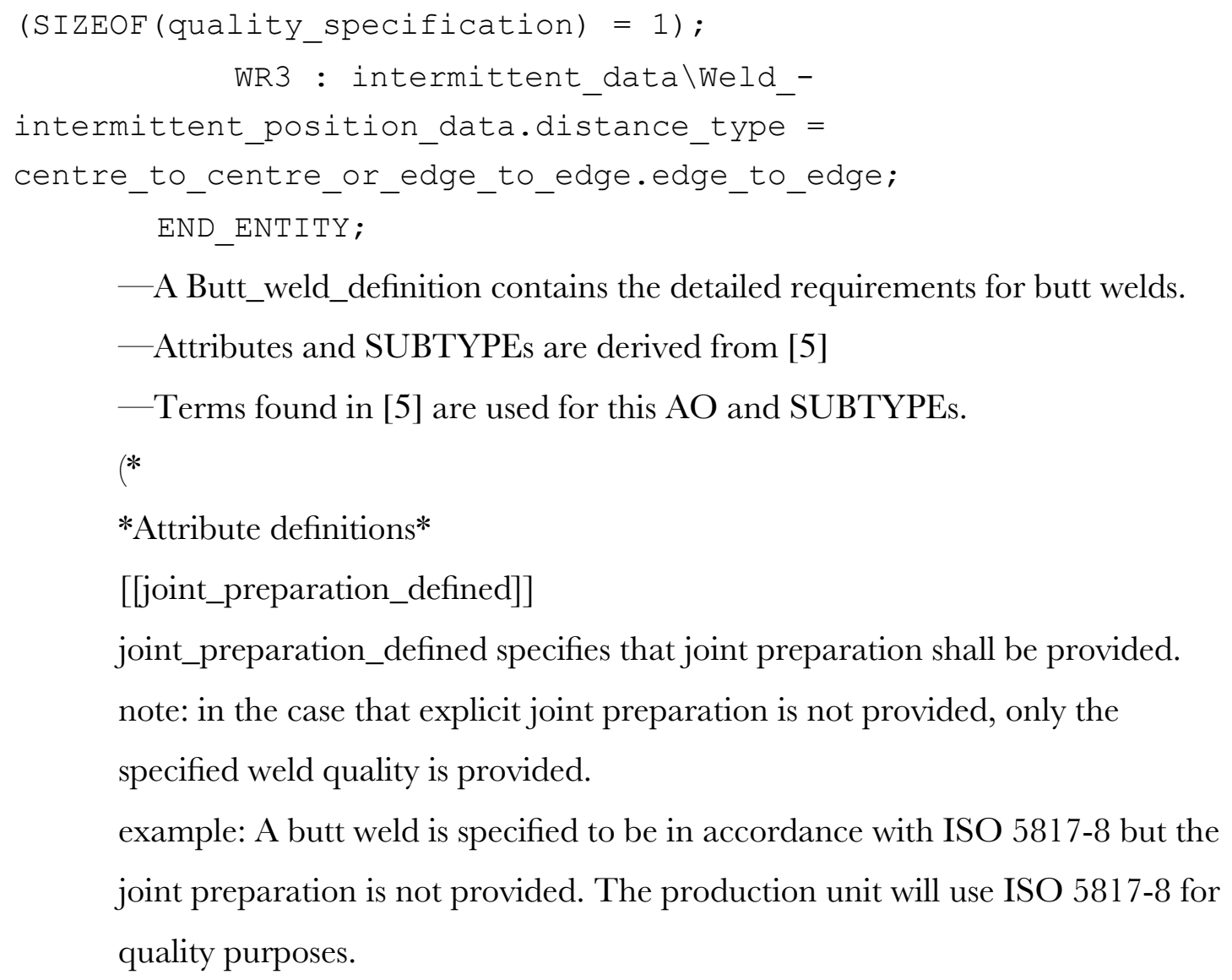

The penetration provided shall be indicated as full or a value shall be provided for partial penetration, but both full indication and partial penetration data shall not be simultaneously provided.

WR2

When joint preparation is defined, a quality specification need not be provided.

When joint preparation is not defined, a quality specification shall be provided.

WR3

The distance type is edge to edge.*)

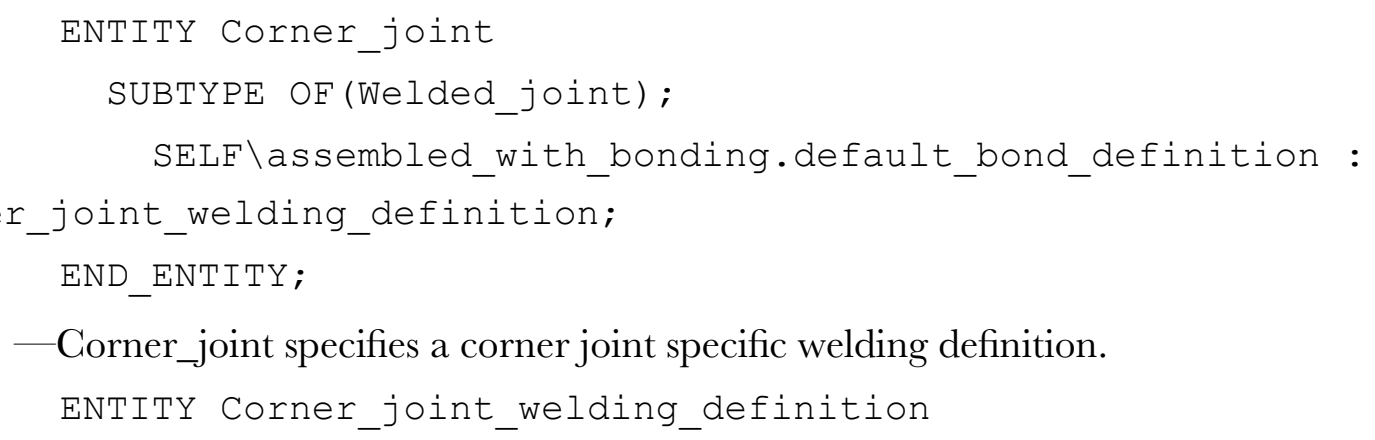

_Corner_joint specifies a corner joint specific welding definition. ENTITY Corner_joint_welding_definition 


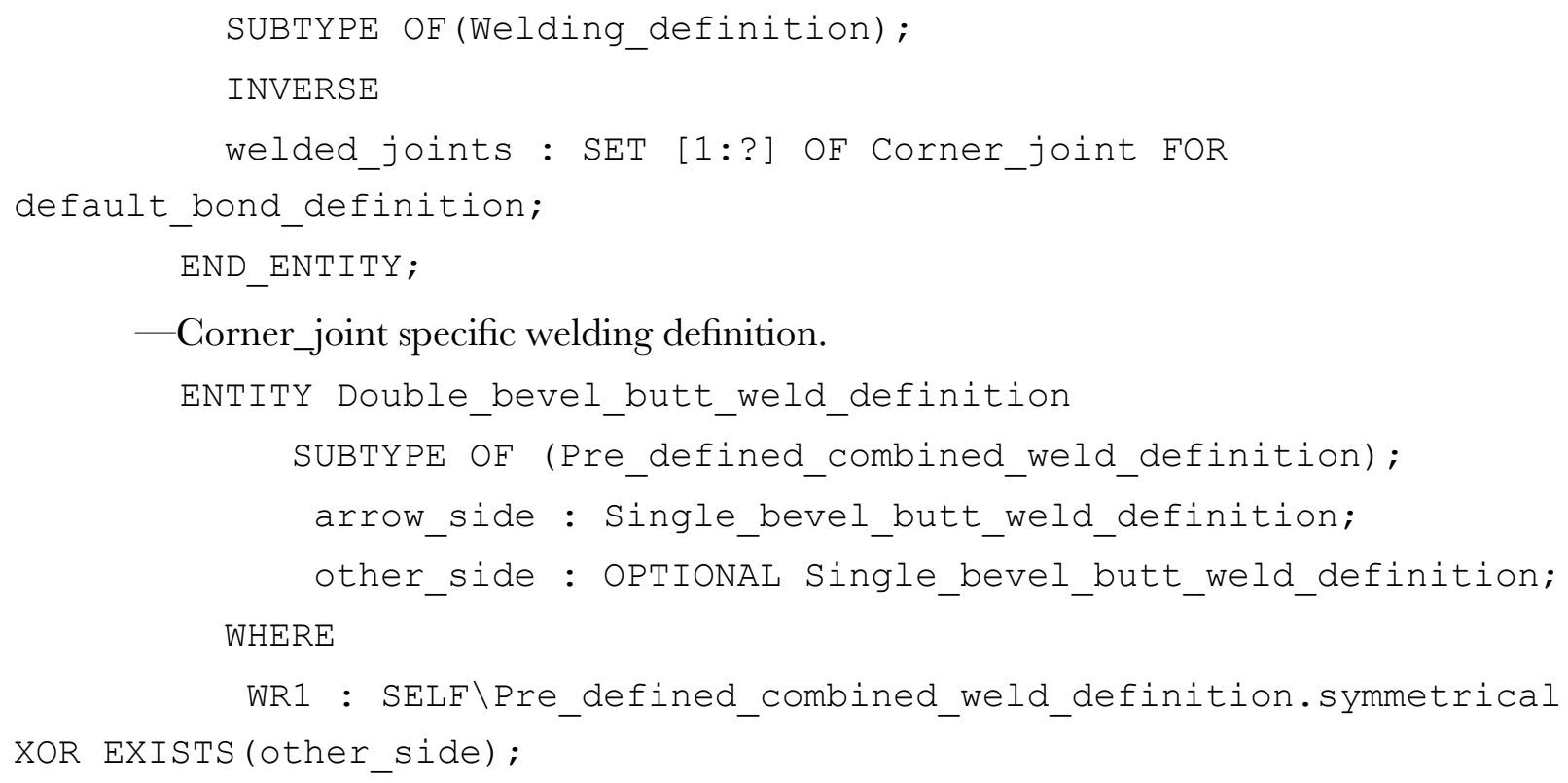

- If the weld is symmetrical then the other side shall not be provided, and the converse is true. END_ENTITY;

- The Double_bevel_butt_weld_definition is specified in [5].

ENTITY Double_bevel_butt_with_broad_root_face_and_fillet_welds_weld_definition

SUBTYPE OF (Double_bevel_butt_weld_definition);

arrow_side_fillet : fillet_weld_definition;

other_side_fillet : OPTIONAL fillet_weld_definition; WHERE

WR1 : SELF $\backslash$ Pre_defined_combined_weld_definition.symmetrical XOR EXISTS(other_side_fillet);

- If the weld is symmetrical then the other side shall not be provided, and the converse is true. END_ENTITY;

—The Double_bevel_butt_with_broad_root_face_and_fillet_welds_weld_definition is specified in [5].

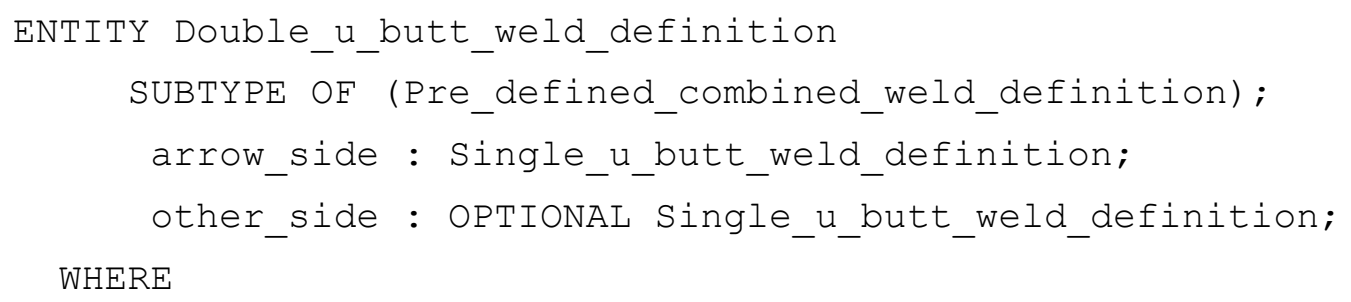


WR1 : SELF $\backslash$ Pre_defined_combined_weld_definition.symmetrical XOR EXISTS(other_side);

- If the weld is symmetrical then the other side shall not be provided, and the converse is true.

END ENTITY;

-The Double_u_butt_weld_definition is specified in [5].

ENTITY Double_v_butt_weld_definition

SUBTYPE OF (Pre_defined_combined_weld_definition);

arrow_side : Single_v_butt_weld_definition;

other_side : OPTIONAL Single_v_butt_weld_definition;

WHERE

WR1 : SELF $\backslash$ Pre_defined_combined_weld_definition.symmetrical

XOR EXISTS(other_side);

- If the weld is symmetrical then the other side shall not be provided, and the converse is

true.

END_ENTITY;

-The Double_v_butt_weld_definition is specified in [5].

ENTITY Edge_joint

SUBTYPE OF (Welded_joint);

SELF\assembled_with_bonding.default_bond_definition :

Edge_joint_welding_definition;

END_ENTITY;

_Edge_joint specifies an edge joint specific welding definition.

ENTITY Edge_joint_welding_definition SUBTYPE

OF(Welding_definition);

INVERSE

welded_joints : SET [1:?] OF Edge_joint FOR

default_bond_definition;

END ENTITY;

_Edge_joint specific welding definition.

ENTITY Edge_weld_definition

SUBTYPE OF(Weld_definition);

weld_metal_thickness : Length_tolerance_characteristic;

- The metal thickness is a length and may have a tolerance.

END_ENTITY; 
- The edge weld is specified in [5].

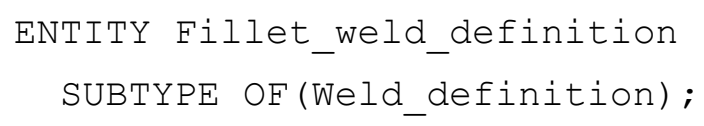

- The distance between welds in the intermittent case shall be edge to edge. END_ENTITY;

- The fillet weld is specified in [5].

- Leg length, nominal throat thickness, deep penetration throat thickness, and intermittent data are specified in [5].

- Unequal legs specifies two surface based properties that when provided, are provided in pairs as specified in [5].

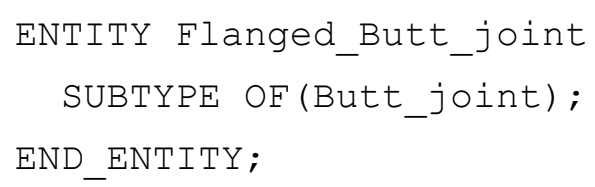

-A type of butt joint specified in [5].

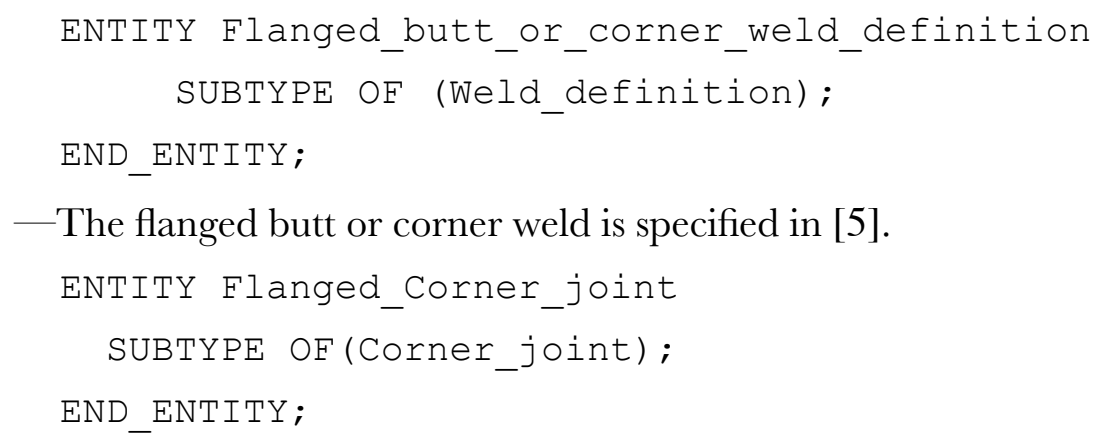

- The flanged butt or corner weld is specified in [5]. 
-A type of corner joint specified in [5].

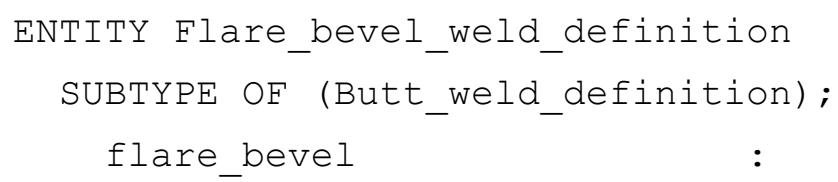

-Flare bevel is a length specified in [5] that may have a tolerance applied. END_ENTITY;

-A type of butt weld specified in [5].

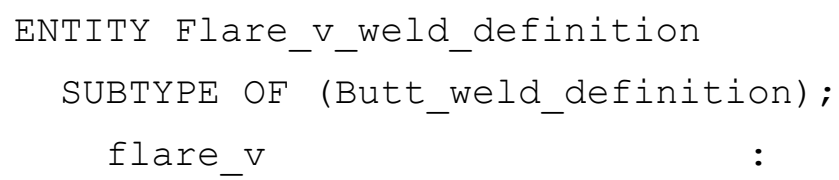

- Flare v is a length specified in [5] that may have a tolerance applied. END_ENTITY;

-A type of butt weld specified in [5].

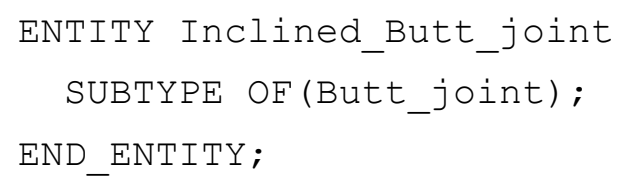

-A type of butt joint specified in [5].

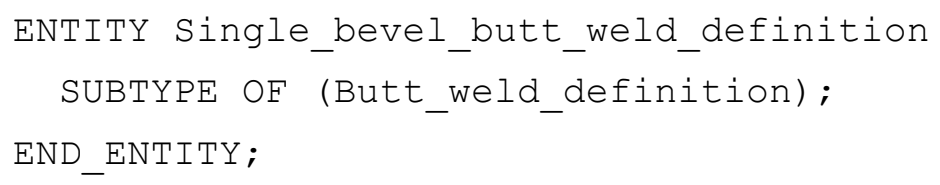

-A type of butt weld specified in [5].

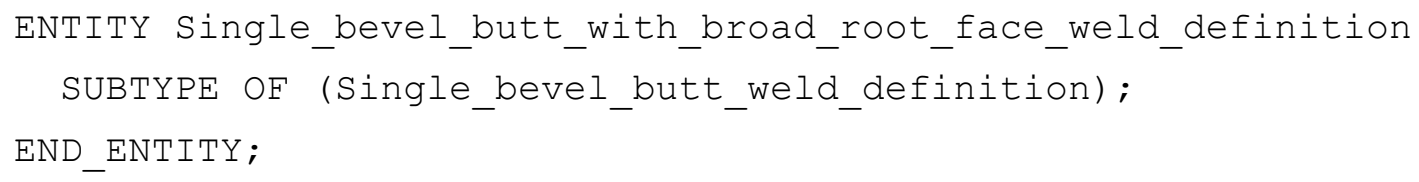

-A type of butt weld specified in [5].

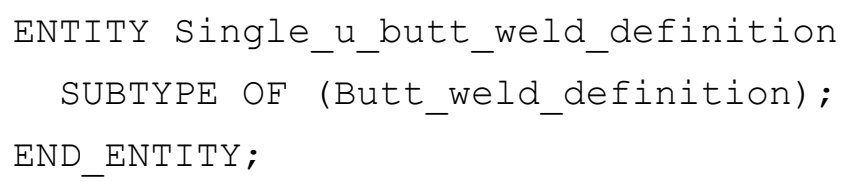

-A type of butt weld specified in [5].

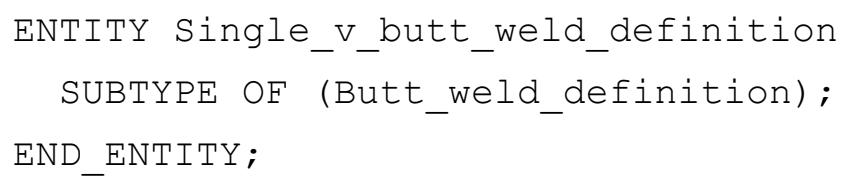


- A type of butt weld specified in [5].

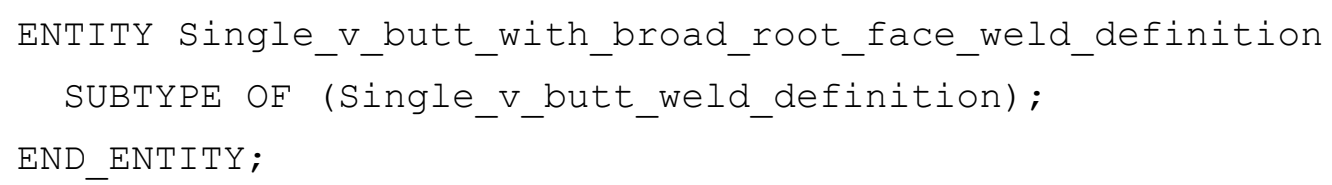

- A type of butt weld specified in [5].

ENTITY Lap_joint

SUBTYPE OF(Welded_joint);

SELF \assembled_with_bonding.default_bond_definition :

Lap_joint_welding_definition;

END_ENTITY;

—Lap_joint specifies a lap joint specific welding definition.

ENTITY Lap_joint_welding_definition

SUBTYPE OF (Welding_definition);

INVERSE

welded_joints : SET [1:?] OF Lap_joint FOR

default_bond_definition;

END_ENTITY;

_Lap_joint specific welding definition.

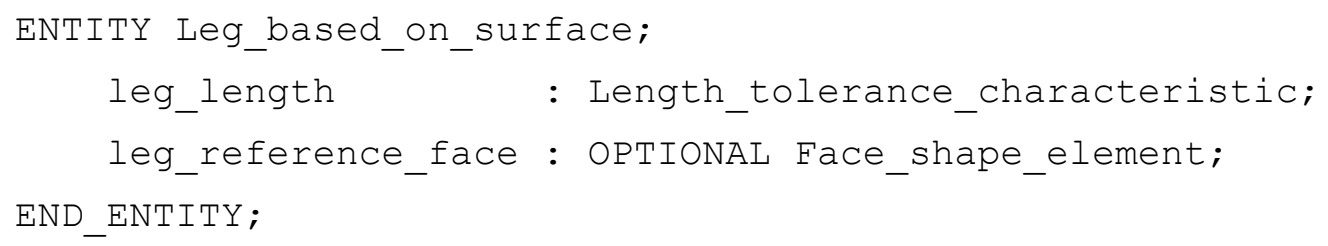

-A Leg_based_on_surface associates a length characteristic with a face. This is provided as a separate $\mathrm{AO}$ in order to improve ARM clarity.

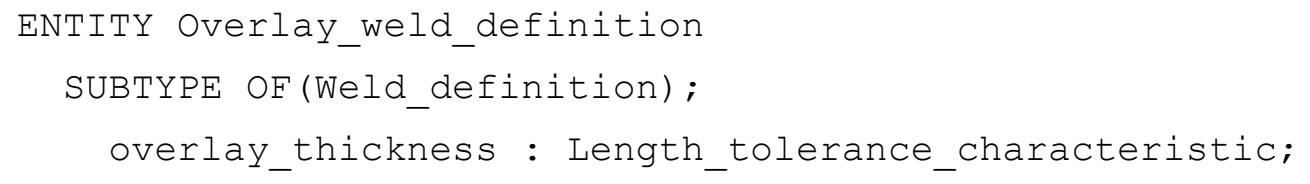

- Thickness specified in [5]. The length may have a tolerance applied. END_ENTITY;

-A type of weld specified in [5].

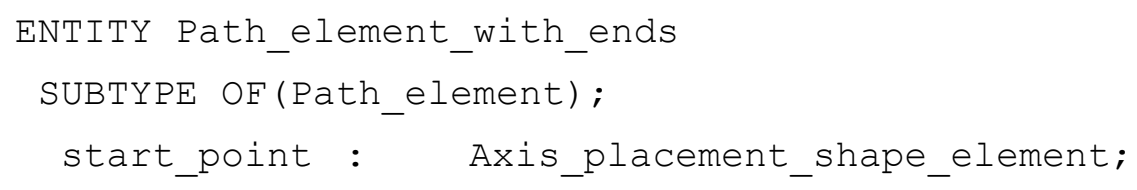

- The start is associated with an Axis_placement.

$$
\text { end_point : Axis_placement_shape_element; }
$$


- The end is associated with an Axis_placement.

WHERE

WR1 : start_point :<>: end_point;

- The start and end points are different.

END_ENTITY;

-Path_element_with_ends is provided when needed. Explicit ends are provided.

ENTITY Plug_weld_in_circular_holes_definition

SUBTYPE OF (Weld_definition);

full_penetration : BOOLEAN;

plug_diameter :

Length_tolerance_characteristic;

partial_fill_depth : OPTIONAL

Length_tolerance_characteristic;

intermittent_data : OPTIONAL

Weld_intermittent_position_data;

WHERE

WR1 : intermittent_data \Weld_intermittent_-

position_data.intermittent_type = intermittent_or_chain_intermittent_or_staggered_intermittent.intermittent;

-Only intermittent data shall be provided for

Plug_weld_in_slot_definition.intermittent_data.

WR2 : intermittent_data \Weld_intermittent_-

position_data.distance_type = centre_to_-

centre_or_edge_to_edge.centre_to_centre;

- The distance between weld elements shall be centre to centre.

WR3 : full_penetration XOR EXISTS(partial_fill_depth);

--The penetration provided shall be indicated as full or a value shall be

--provided for partial fill, but both full indication and partial

--fill data shall not be simultaneously provided.

END_ENTITY;

-A type of weld specified in [5].

ENTITY Plug_weld_in_slot_definition

SUBTYPE OF (Weld_definition);

full_penetration : BOOLEAN;

slot_width : OPTIONAL

On Extending an ISO Standard for Exchanging Product Manufacturing Information

Page 51 


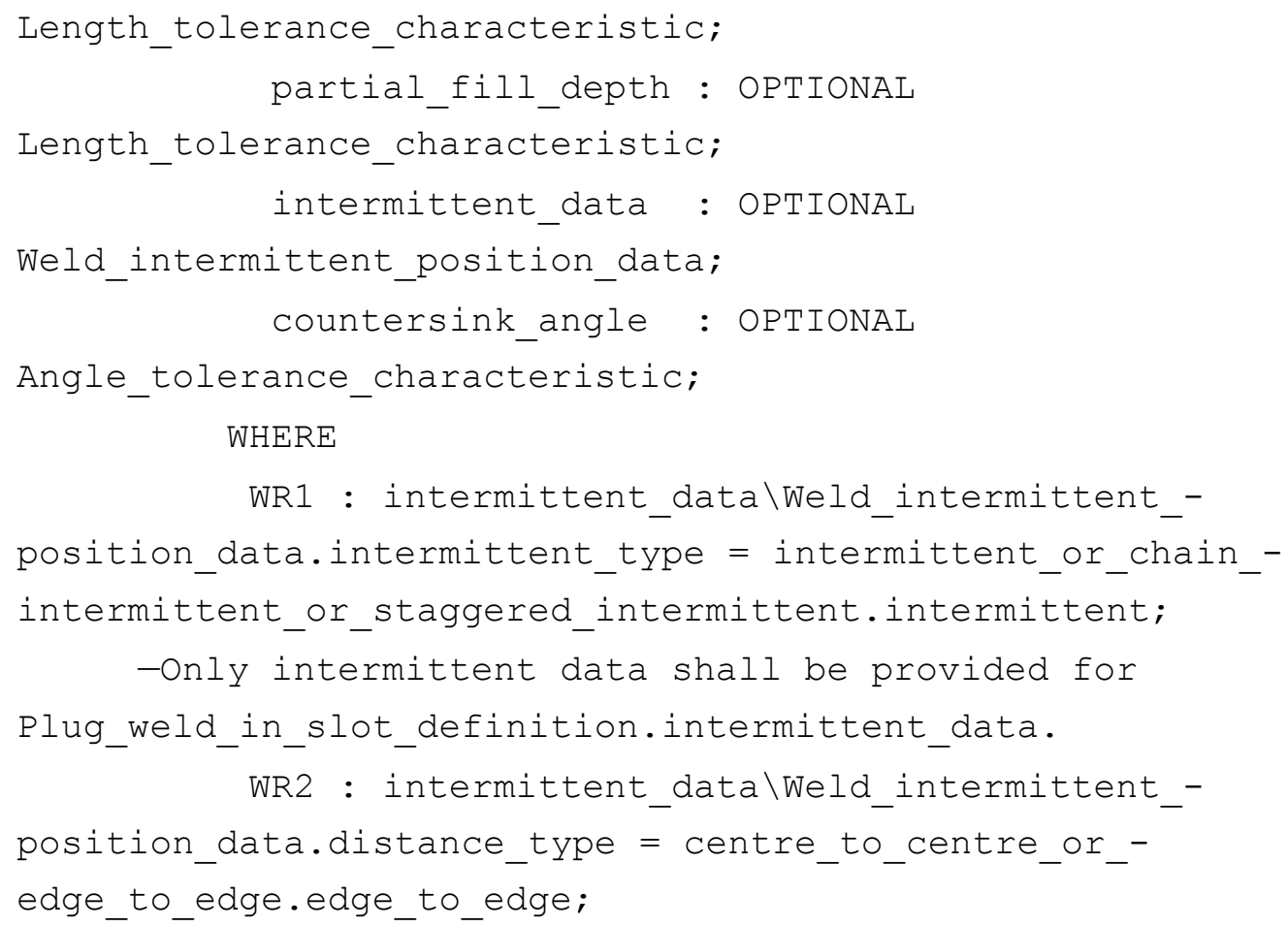

- The distance between weld elements shall be edge to edge.

WR3 : full_penetration XOR EXISTS(partial_fill_depth);

--The penetration provided shall be indicated as full or a value shall be provided for partial fill, but both full indication and partial fill data shall not be simultaneously provided. END ENTITY;

- A type of weld specified in [5].

- Full penetration, slot width, partial fill depth, intermittent data, and countersink angle are specified in [5].

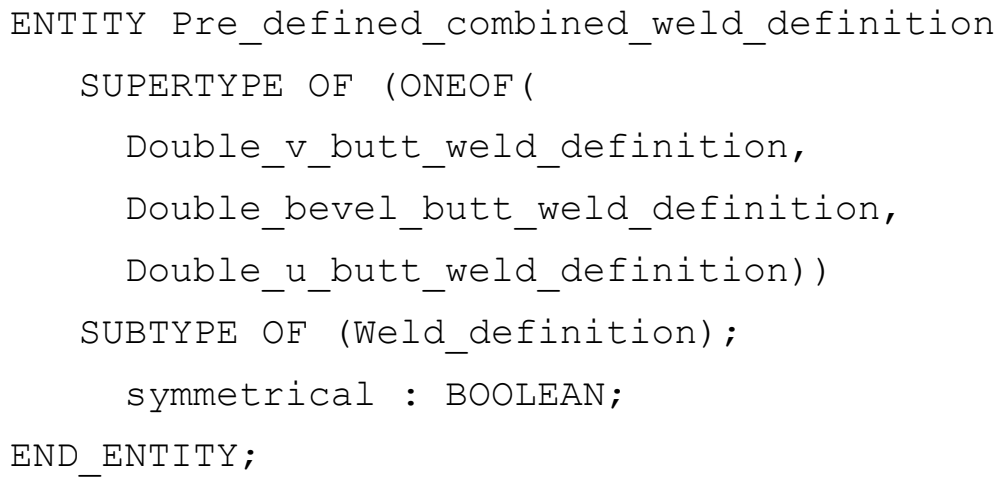

- Predefined weld combinations specified in [5].

- Symmetrical specifies if both arrow and other side are treated equally. ENTITY Seam_weld_definition 
SUBTYPE OF (Weld_definition);

$$
\text { weld_type : resistance_or_fusion_weld; }
$$

-A weld may be either resistance or fusion.

$$
\text { intermittent_data : OPTIONAL }
$$

Weld_intermittent_position_data;

- There may be more than one weld specified.

$$
\text { weld_width : Length_tolerance_characteristic; }
$$

- The cross-sectional width of the weld.

WHERE

WR1 : intermittent_data \Weld_intermittent_-

position_data.intermittent_type = intermittent_or_chain_-

intermittent_or_staggered_intermittent.intermittent;

-Only intermittent data shall be provided for

Plug_weld_in_slot_definition.intermittent_data.

$$
\text { WR2 : intermittent_data \Weld_intermittent_- }
$$

position_data.distance_type = centre_to_centre_-

or_edge_to_edge.edge_to_edge;

- The distance between weld elements shall be edge to edge.

END_ENTITY;

- A type of weld specified in [5].

ENTITY Single_j_butt_weld_definition

SUBTYPE OF (Butt_weld_definition);

END_ENTITY;

- A type of weld specified in [5].

ENTITY Spot_weld_definition

SUBTYPE OF (Weld_definition);

weld_diameter : Length_tolerance_characteristic;

intermittent_data : OPTIONAL

Weld_intermittent_position_data;

- There may be more than one weld specified.

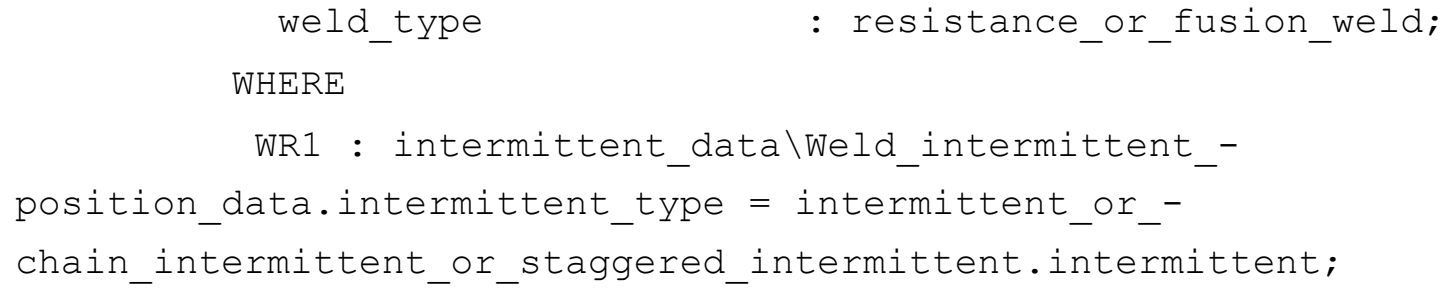


- Only intermittent data shall be provided for

Plug_weld_in_slot_definition.intermittent_data.

WR2 : intermittent_data \Weld_intermittent_-

position_data.distance_type = centre_to_centre_or_-

edge_to_edge.centre_to_centre;

- The distance between weld elements shall be centre to centre.

IP1 : The nominal weld_diameter shall equal intermittent_data \Weld_intermittent_position_data.nominal_length_of_weld_element.

END ENTITY;

- A type of weld specified in [5].

-Weld diameter, intermittent data, and weld type are specified in [5].

ENTITY Square_butt_weld_definition

SUBTYPE OF (Butt_weld_definition);

END_ENTITY;

-A type of weld specified in [5].

ENTITY Stake_weld_definition

SUBTYPE OF (Weld_definition);

END_ENTITY;

-A type of weld specified in [5].

ENTITY Steep_flanked_single_v_butt_weld_definition

SUBTYPE OF (Single_v_butt_weld_definition);

END_ENTITY;

-A type of weld specified in [5].

ENTITY Stud_weld_definition

SUBTYPE OF(Weld_definition);

stud_diameter : Length_tolerance_characteristic;

intermittent_data : OPTIONAL

Weld_intermittent_position_data;

WHERE

WR1 : intermittent_data \Weld_intermittent_-

position_data.intermittent_type = intermittent_or_-

chain_intermittent_or_staggered_intermittent.intermittent;

-Only intermittent data shall be provided for

Plug_weld_in_slot_definition.intermittent_data.

On Extending an ISO Standard for Exchanging Product Manufacturing Information

Page 54 


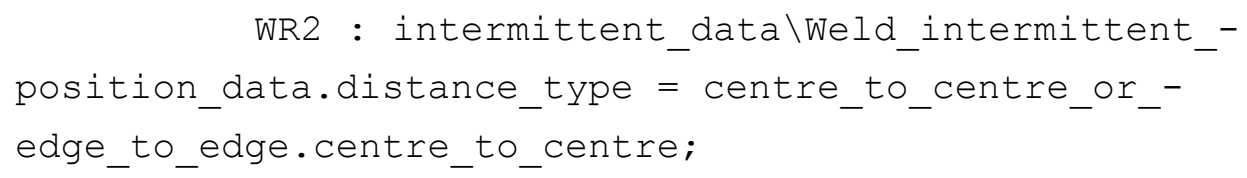

- The distance between weld elements shall be centre to centre.

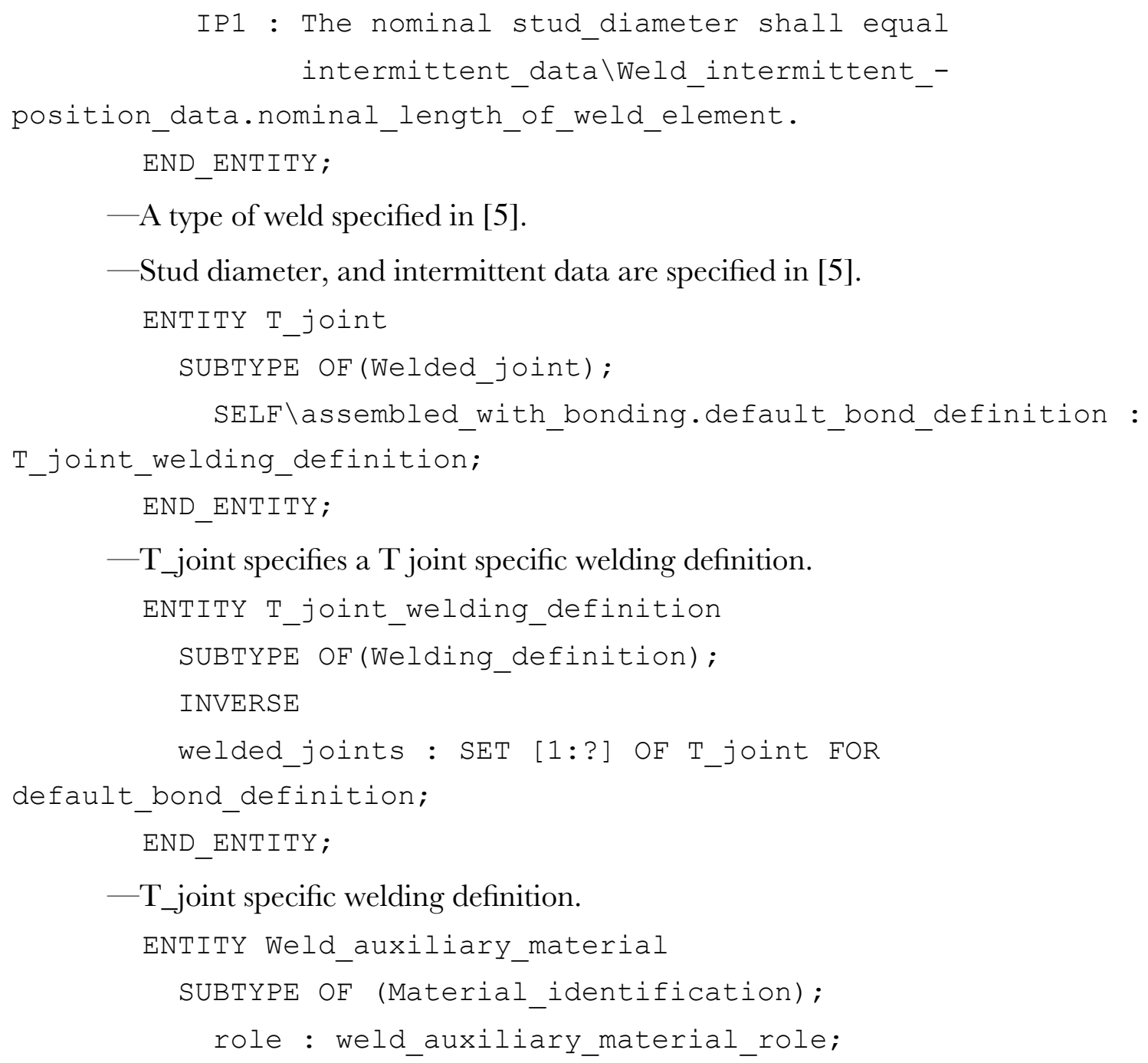

- A type of weld specified in [5].

- Stud diameter, and intermittent data are specified in [5].

ENTITY T_joint

- $\mathrm{T}_{-}$joint specifies a $\mathrm{T}$ joint specific welding definition.

-Role as defined in [4] or [5]

shape : OPTIONAL Associated_shape_element;

- There may be a specific shape provided.

SELF \Material_identification.items : SET [1:1] OF Welding_definition;

- The Welding_definition for which the material is auxiliary. END_ENTITY;

- Material used as auxiliary material. This is the standard STEP material model. ENTITY Weld_context 


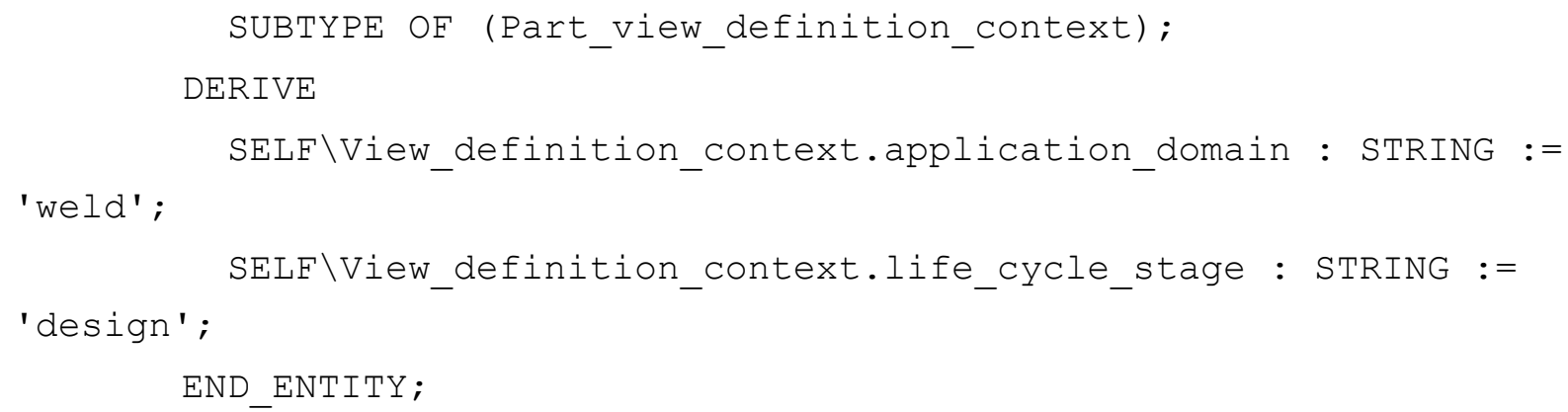

-A view definition context is provided to allow validation, verification and conformance support.

- This context is a design context and a weld context.

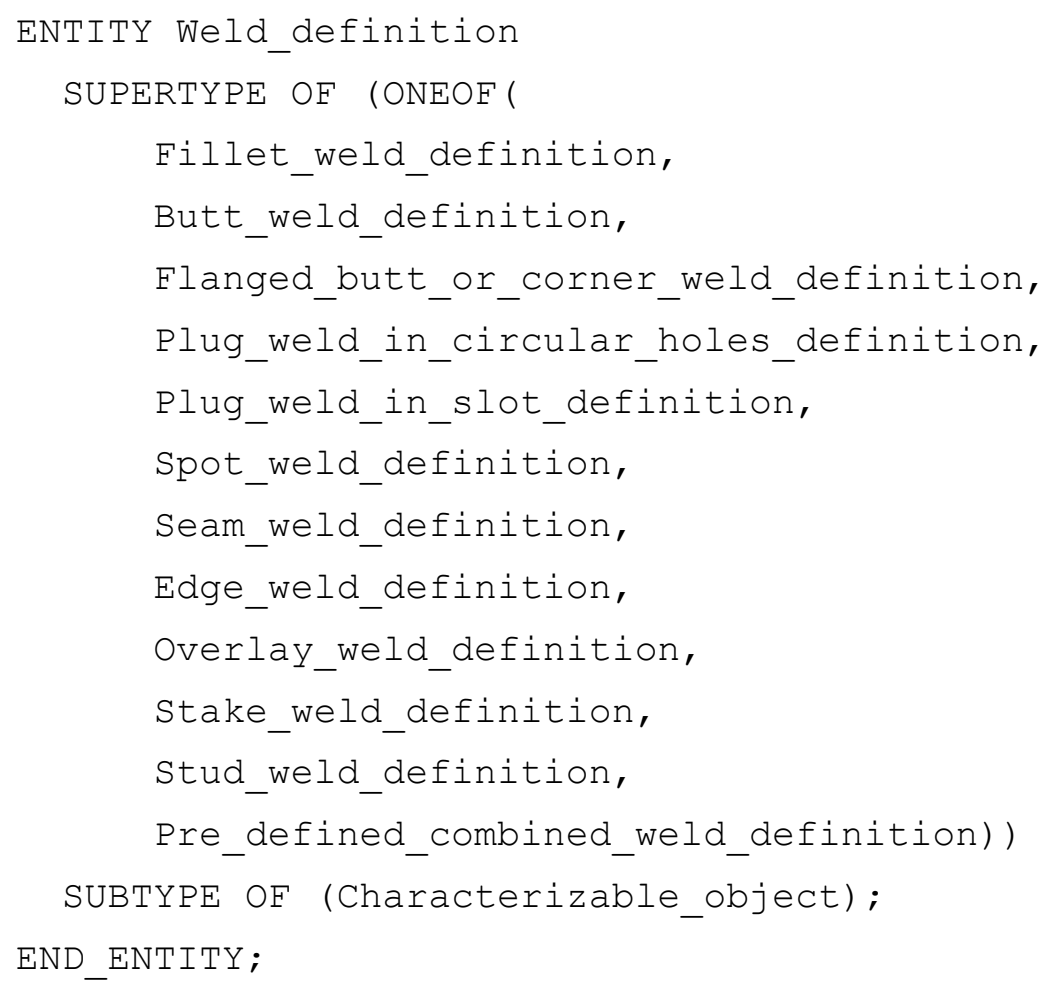

- The weld types as specified in [4] and [5]. Some types are further SUBTYPEd in this document in accordance with [4] and [5].

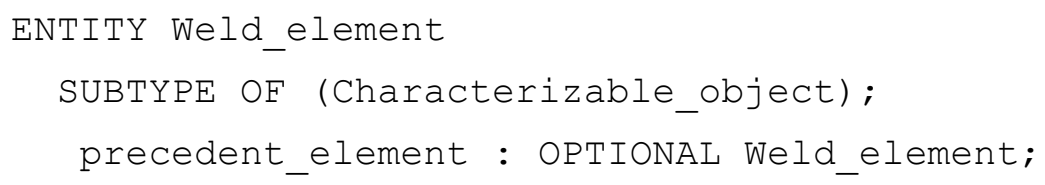

- The preceding element in the linked list of elements.

$$
\text { identifier : count_measure; }
$$

- The identification of the position of this element in the list.

$$
\text { side : arrow_or_other; }
$$


- The side to which the weld element is applied.

derived_from : Weld_intermittent_position_data;

- The parametric source for identification of weld elements. There is also an existence dependency relationship on the Weld_intermittent_position_data that is in the role of derived_from.

\section{INVERSE}

subsequent_element : SET [0:1] OF Weld_element for

precedent_element;

- In a linked list, the subsequent element.

UNIQUE

UR1 : identifier, derived_from;

- No identifier may be used more than once in the context of a specific

Weld_intermittent_position_data.

WHERE

WR1 : identifier $>0$;

- The lower bound of the identifier shall be 1 .

WR2 : identifier <= derived_from \Weld_intermittent_-

position_data.number_of_weld_elements;

- The upper bound of the identifier shall be the number of Weld_elements.

WR3 : NOT EXISTS (precedent_element) OR

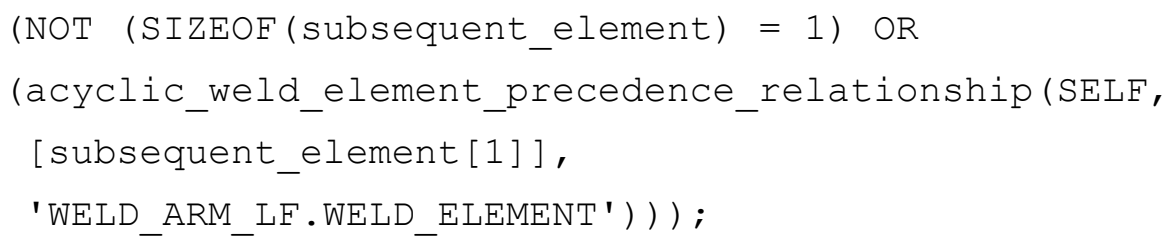

- There shall be no cycles in the list of Weld_elements.

WR4 : EXISTS (precedent_element) XOR (identifier = 1);

- The initial element shall provide no precedent_element; for that initial element the identifier shall be 1 .

END_ENTITY;

(*Weld_element is derived from the Weld_intermittent_position_data as required for inspection purposes. The Weld_element.identifier represents the element at the identifier position in the list of elements.

Weld_element is a requirement specification for a capability. Other solutions may be adopted as a result of the consensus development process in ISO TG 184/SG 4/WG 12. 
*)

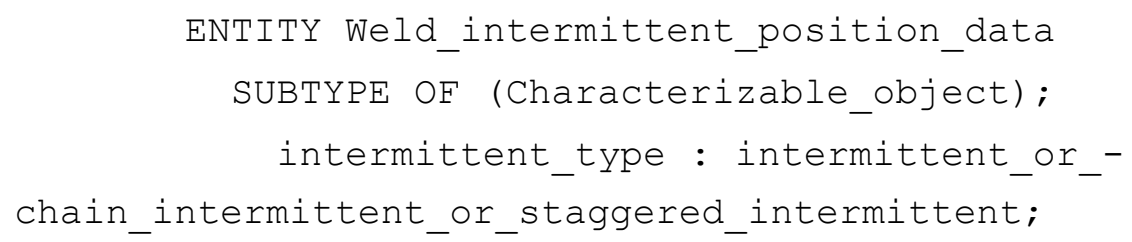

- The position data shall be one of three possible types: intermittent, chained intermittent, or staggered intermittent as specified in [4] and [5]

$$
\text { number_of_weld_elements : count_measure; }
$$

- The number of weld elements as specified in [4] and [5]

$$
\text { nominal_length_of_weld_element : }
$$

Length_data_element;

- The nominal length of a weld element as specified in [4] and [5]

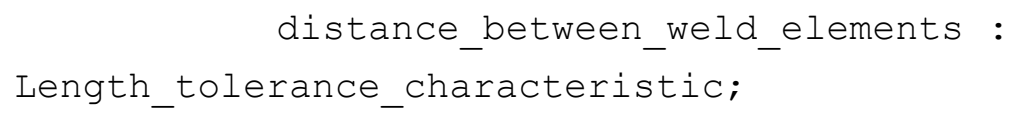

- The distance between weld elements as specified in [4] and [5]

$$
\text { distance_type : centre_to_centre_or_edge_to_edge; }
$$

- The AOs referencing this AO specify whether the distance between weld elements is centre to centre or nearest edge to nearest edge.

INVERSE

weld_elements : SET [0:?] OF Weld_element FOR derived_from;

- The weld elements that are derived from the combination of the number of weld elements, the type of position data, and path information inherited from the welded joint. Weld elements are provided to enable inspection results to be correlated back to the original welding symbol but are not required nor specified in [4] and [5].

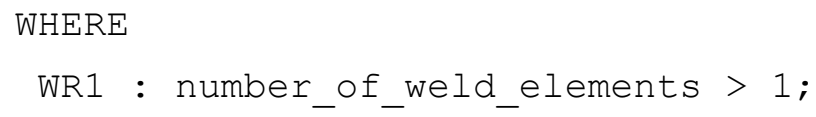

- There shall be at least two weld elements.

$$
\text { WR2 : NOT (SIZEOF(weld_elements) > 0) OR }
$$

$($ SIZEOF (weld_elements) = number_of_weld_elements);

- If the attribute weld_elements is populated with data, then the number of elements in that data shall equal the value of number_of_weld_elements.

$$
\begin{aligned}
\text { WR3 : NOT (SIZEOF (weld_elements) > 0) OR } \\
\text { (SIZEOF (QUERY (we <* weld_elements I EXISTS (we }
\end{aligned}
$$

(Weld_element.precedent_element))) = 


$$
\text { (number_of_weld_elements -1)); }
$$

- If the attribute weld_elements is populated with data, then there shall be one element that does not have a precedent. That element is the initial element in the list.

END_ENTITY;

- The Weld_intermittent_position_data AO is derived from the intermittent data requirements specified in [4] and [5].

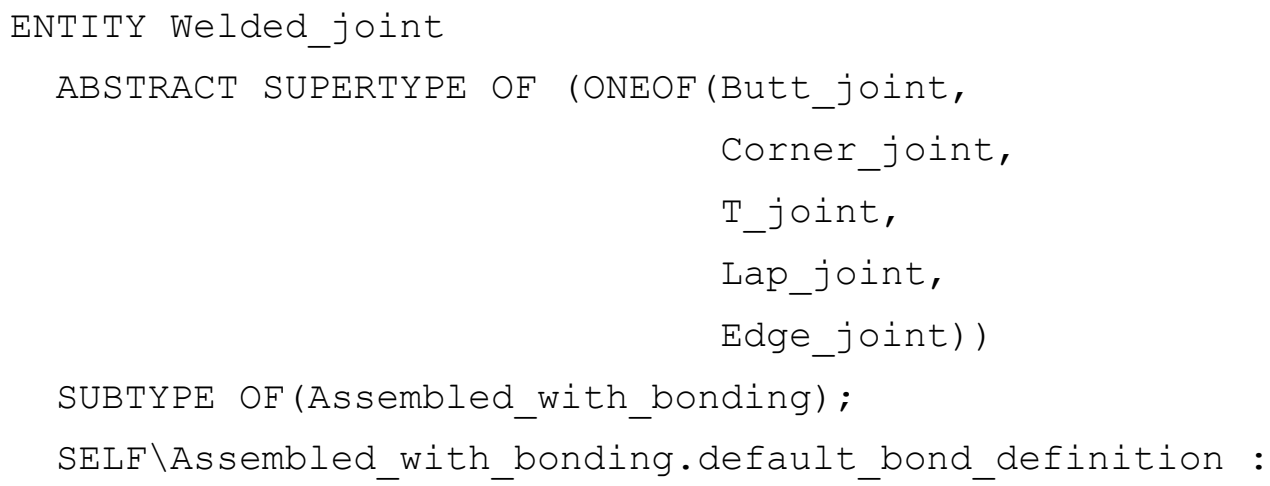

- The Welded_joint is a type of Assembly_joint that is bonded using welding processes.

— The Welded_joint specifies the Welding_definition that provides the details of the welding requirements.

- The types of Welded_joint are specified in [4] and [5].

ENTITY Welding_definition

$$
\begin{aligned}
& \text { ABSTRACT SUPERTYPE OF (ONEOF( } \\
& \text { Butt_joint_welding_definition, } \\
& \text { Corner_joint_welding_definition, } \\
& \text { T_joint_welding_definition, } \\
& \text { Lap_joint_welding_definition, } \\
& \text { Edge_joint_welding_definition)) }
\end{aligned}
$$

- The type of Welding_definition provided is based on the type of Welded_joint associated with the definition.

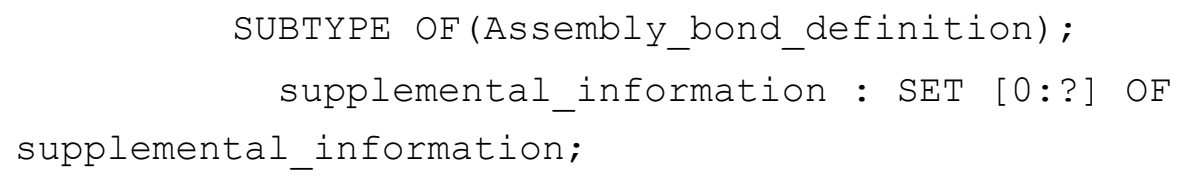

-Optional supplementary information as defined in [4] and [5]

$$
\text { surface_texture : OPTIONAL Surface_texture; }
$$


-Optional surface text information as required by [4] and [5].

$$
\text { welds : SET [1:?] OF Weld_definition; }
$$

-Welds provides the detailed parametric data about each weld defined by a welding symbol.

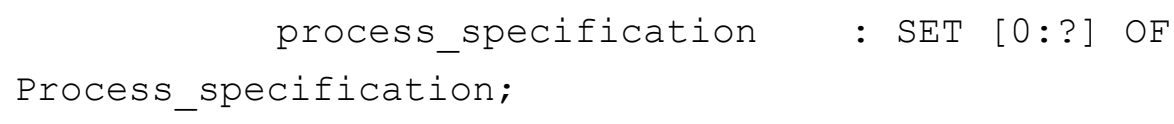

-Optional process specification as required by [4] and [5].

$$
\text { path : OPTIONAL Path_element_with_ends; }
$$

-Optional path for path based welding as required by [4] and [5].

$$
\text { arrow_side_ : Associated_shape_element; }
$$

- The arrow side of the welded joint as defined by [4] and [5].

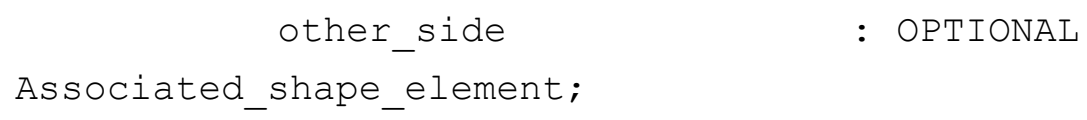

- The other side of the welded joint as defined by [4] and [5].

—The model uses Associated_shape_element to provide a link to a geometric face for both arrow and other side. The model uses a generic reference rather than a specific reference for flexibility in the case that the geometric model does not contain explicit faces.

INVERSE

$$
\text { welded_joint : SET [1:?] OF Welded_joint FOR }
$$

default_bond_definition;

-A Welding_definition may be specified by more than one Welded_joint. That relationship satisfies the requirement in [4] and [5] that a welding symbol shall be able to specify more than one joint.

$$
\text { auxiliary_material : SET [0:?] OF }
$$

Weld_auxiliary_material FOR items;

-Optional material callout as required by [4] and [5].

WHERE

$$
\text { WR1 : arrow_side : }<>\text { : other_side; }
$$

- The arrow side and other side shall be distinct.

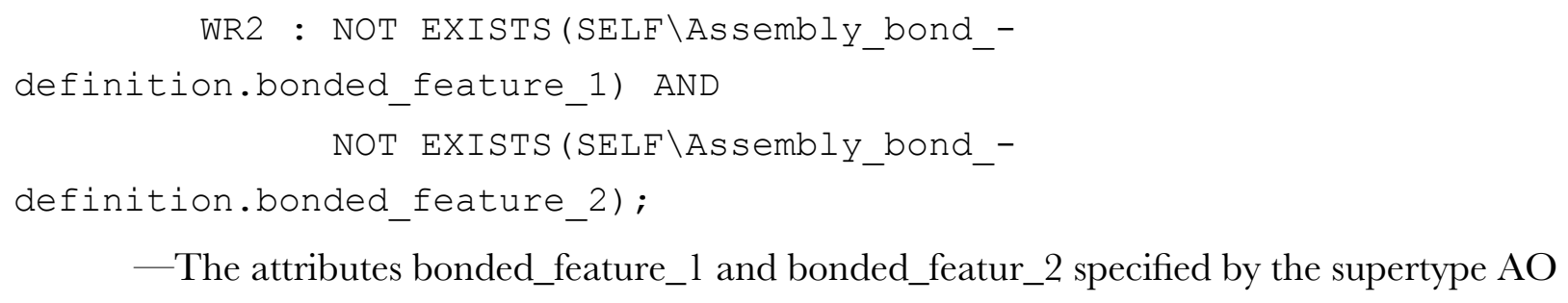

- The attributes bonded_feature_1 and bonded_featur_2 specified by the supertype AO 
Assembly_bond_definition shall not be provided.

END_ENTITY;

ENTITY Welding_definition_sequence_relationship;

precedent_welding_definition : Welding_definition;

subsequent_welding_definition : Welding_definition;

WHERE

WR1 : w_acyclic_sequence (precedent_welding_definition,

subsequent_welding_definition);

- There shall be no cycles in the sequence.

END_ENTITY;

-Welding symbols may be applied in a sequence. This AO provides a directed sequence relationship as defined by [4] and [5].

FUNCTION w_acyclic_sequence ( input1 : Welding_definition;

input2 : Welding_definition) : BOOLEAN;

RETURN (TRUE);

END_FUNCTION;

- w_acyclic_sequence is a function signature provided because ARMs are not formally implemented in STEP.

FUNCTION acyclic_weld_element_precedence_relationship

(relation : Weld_element; relatives : SET[1:?] OF

Weld_element; specific_relation : STRING) : BOOLEAN;

LOCAL

$x$ : SET OF Weld_element;

END_LOCAL;

IF relation.precedent_element IN relatives THEN

RETURN (FALSE);

END_IF;

$\mathrm{x}:=$ QUERY $(\underline{\mathrm{p}} \mathrm{d}<*$

bag_to_set(USEDIN(relation.precedent_element,

'WELD_ARM.' + 'WELD_ELEMENT.' +

'SUBSEQUENT_ELEMENT')) | specific_relation IN

TYPEOF ( $\underline{\mathrm{pd}}))$;

REPEAT $i:=1$ TO $\operatorname{HIINDEX}(\mathrm{x})$;

IF NOT acyclic_weld_element_precedence_relationship(x[i],

relatives + 


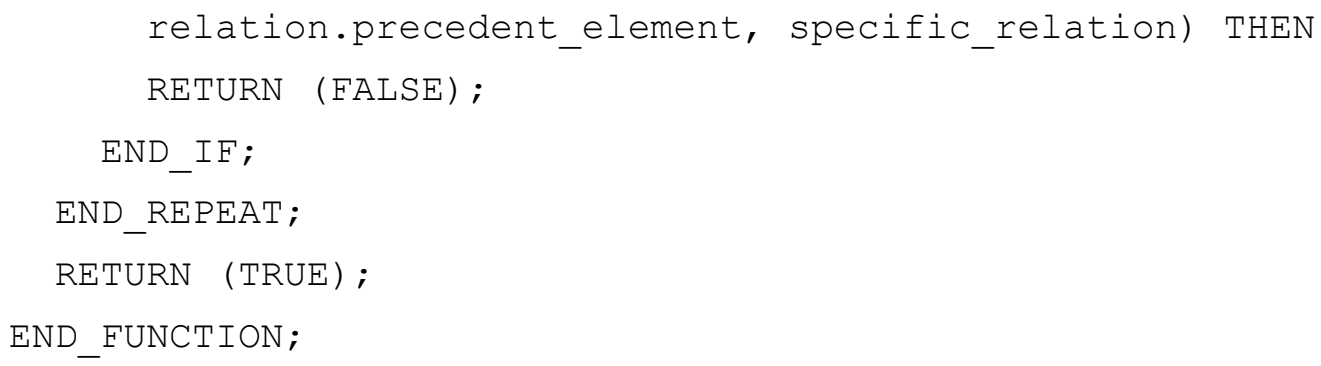

- This function returns TRUE if there are no cycles in the sequence of weld elements. END_SCHEMA; 


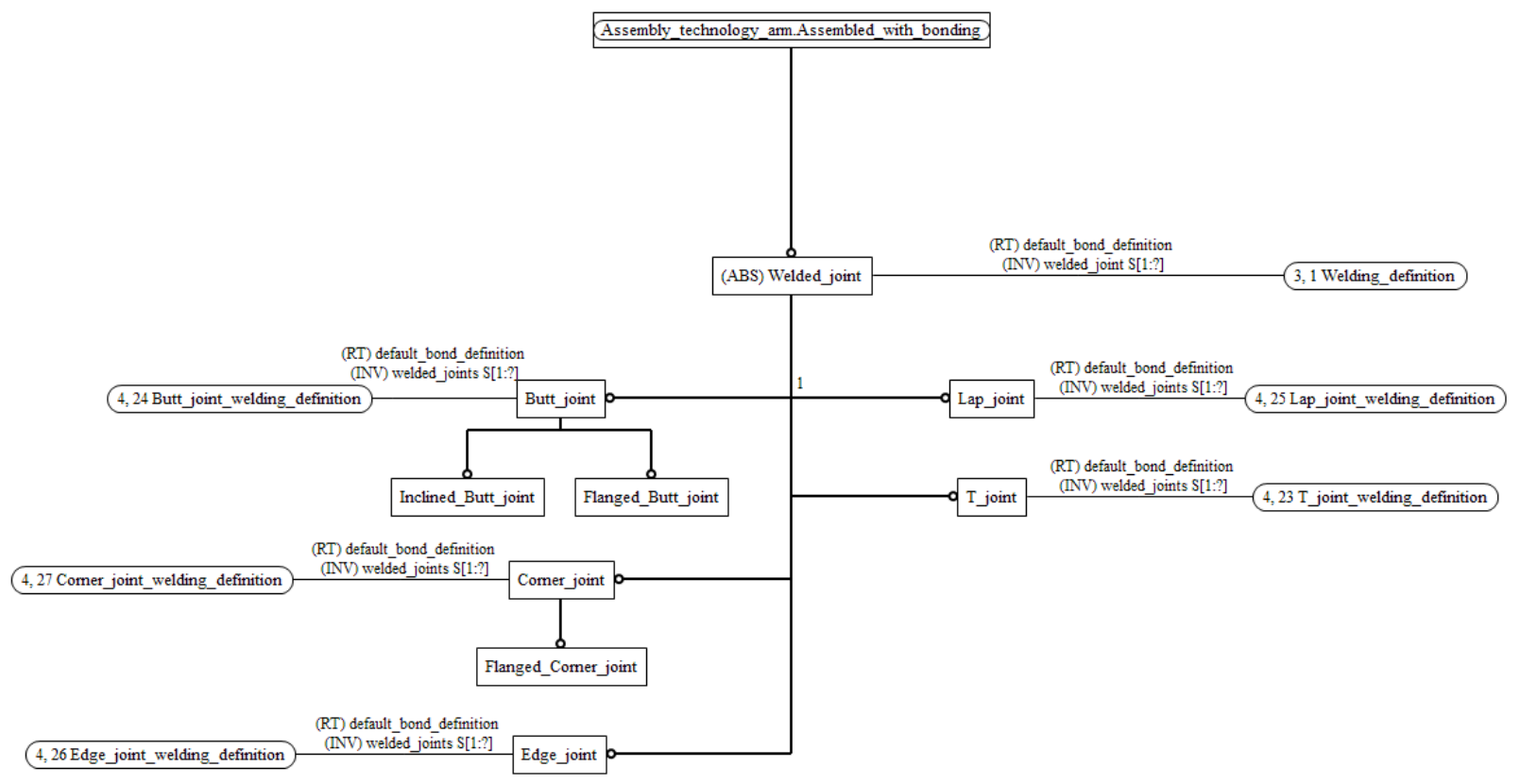

Figure 12 Weld ARM Diagram 1 of 13 
NIST GCR 15-988

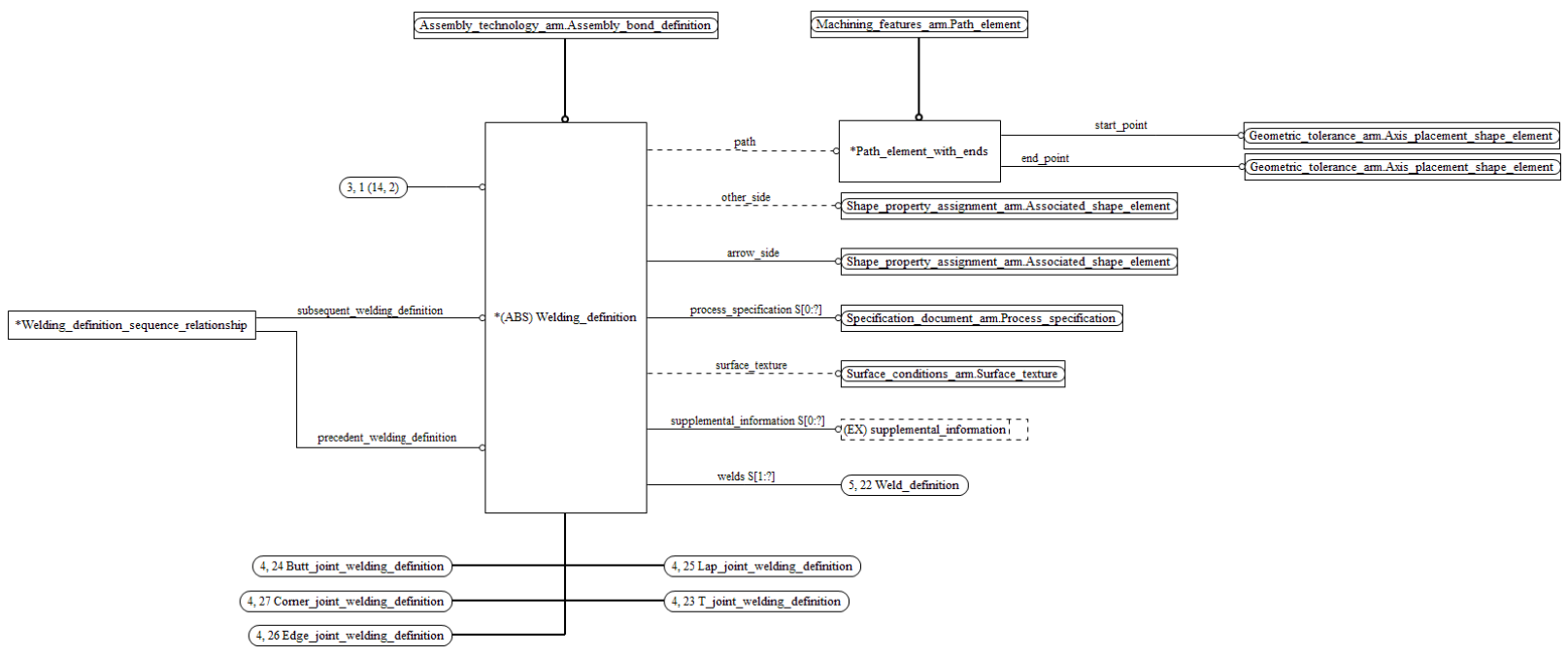

Figure 13 Weld ARM Diagram 2 of 14 

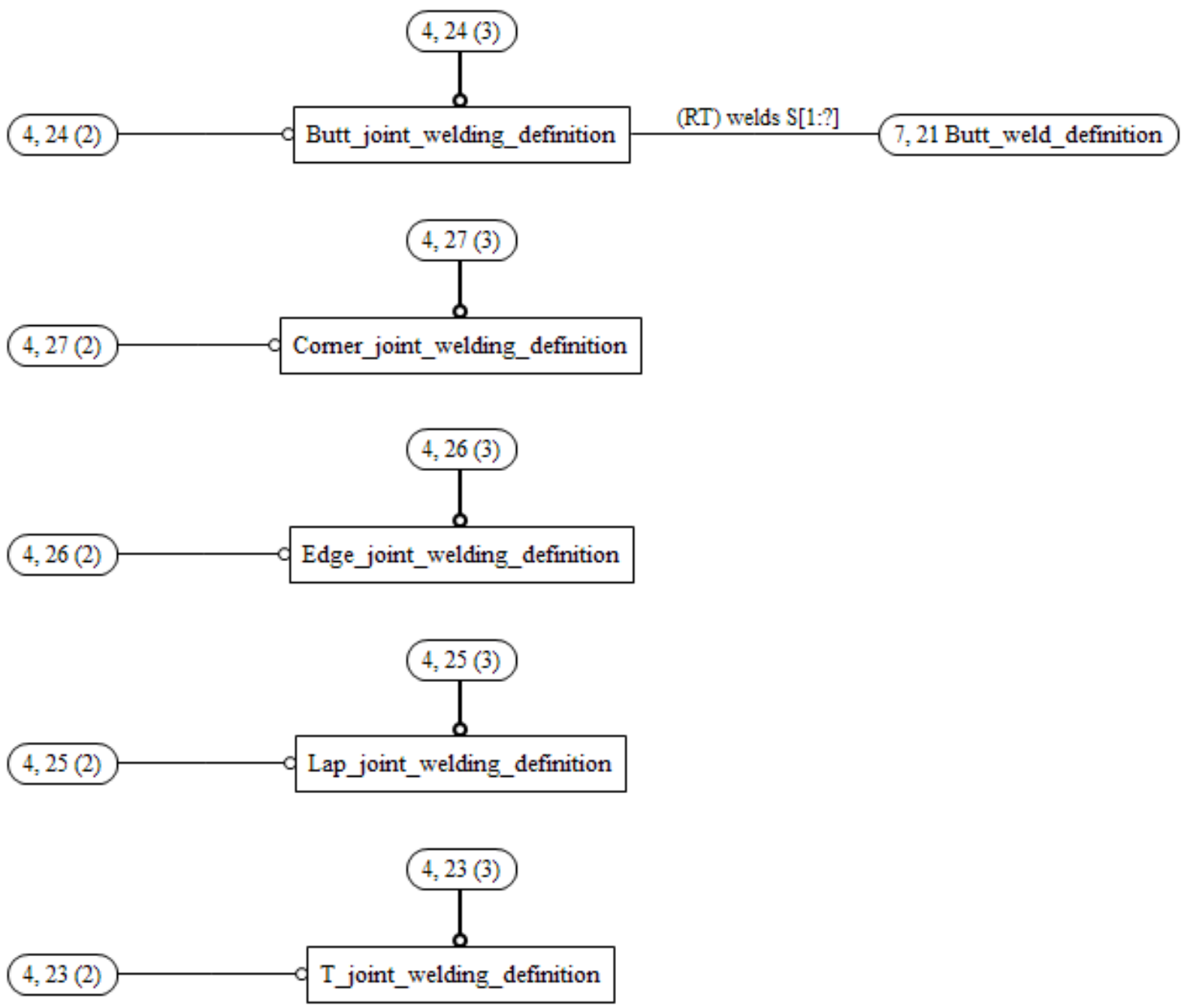

Figure 14 Weld ARM Diagram 3 of 14 


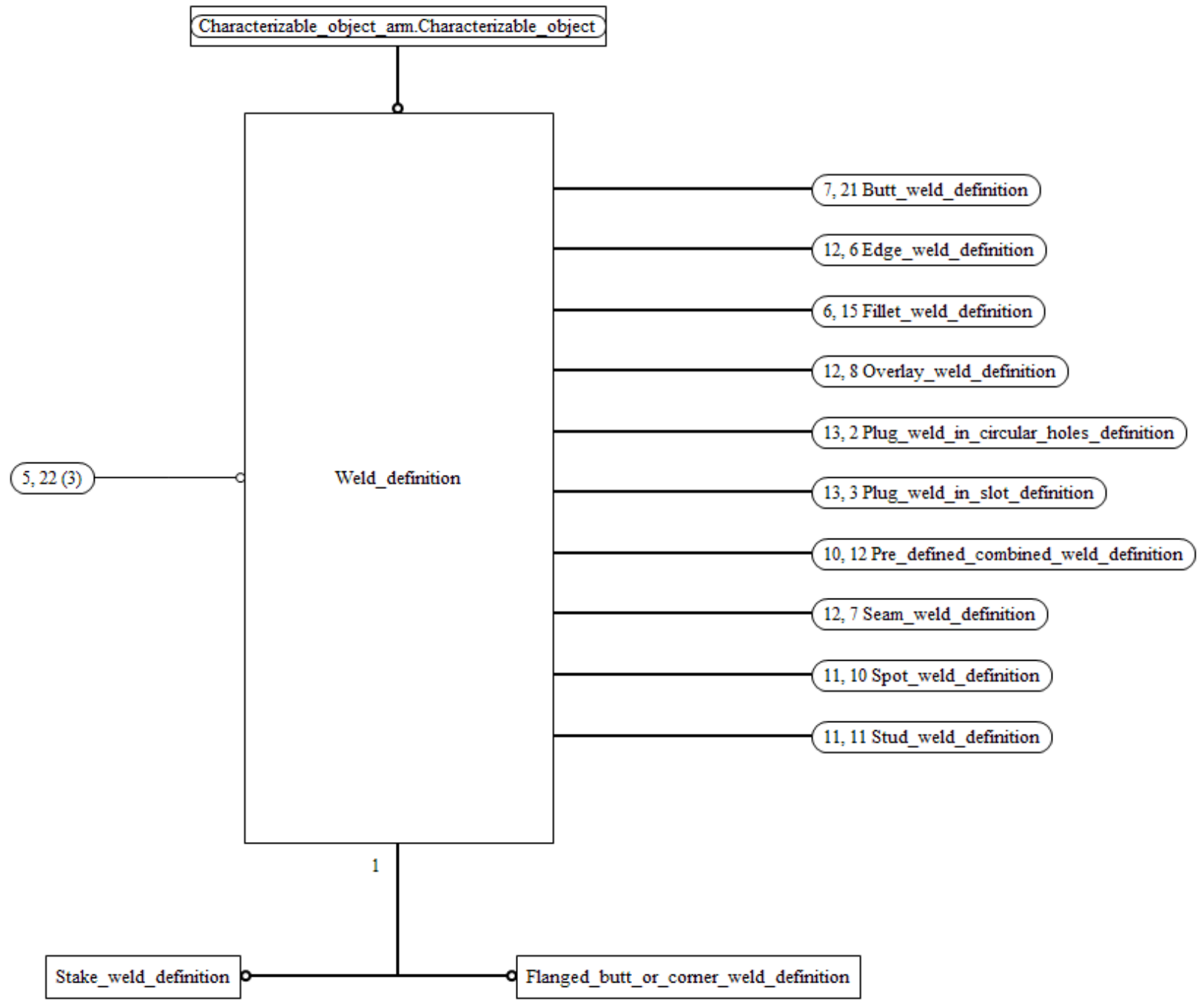

Figure 15 Weld ARM Diagram 4 of 14 


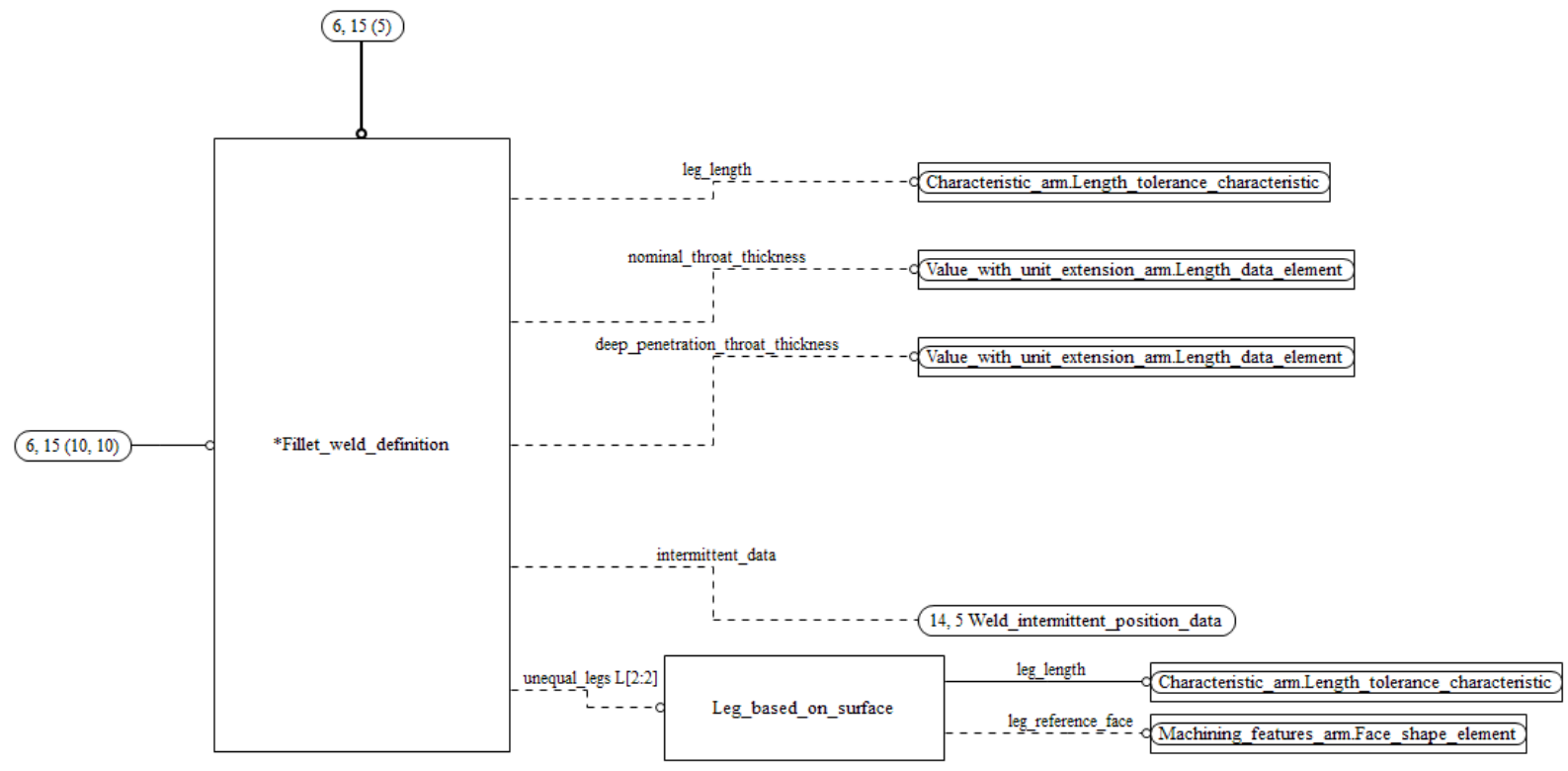

Figure 16 Weld ARM Diagram 5 of 14 

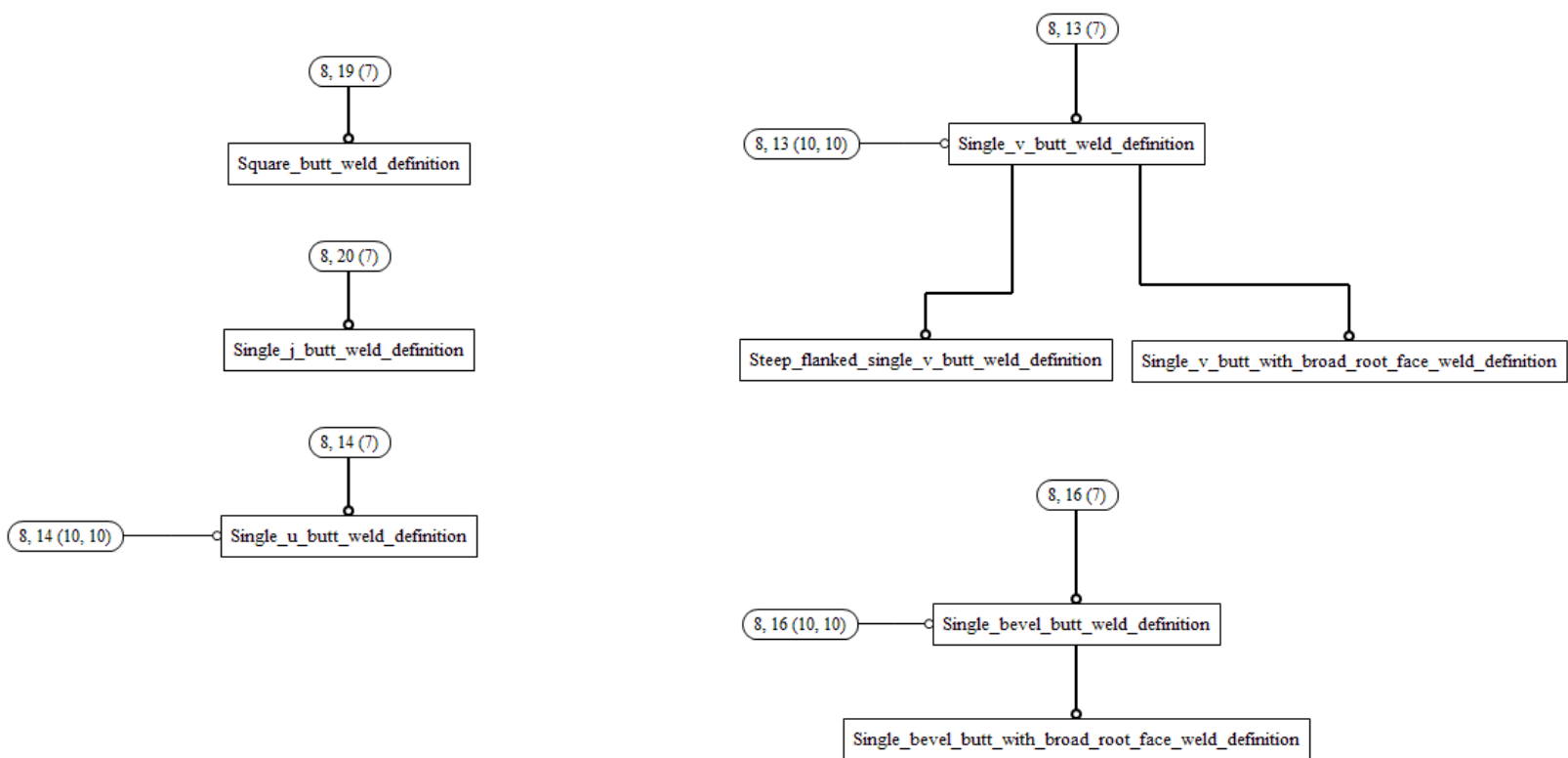

Figure 17 Weld ARM Diagram 6 of 14 


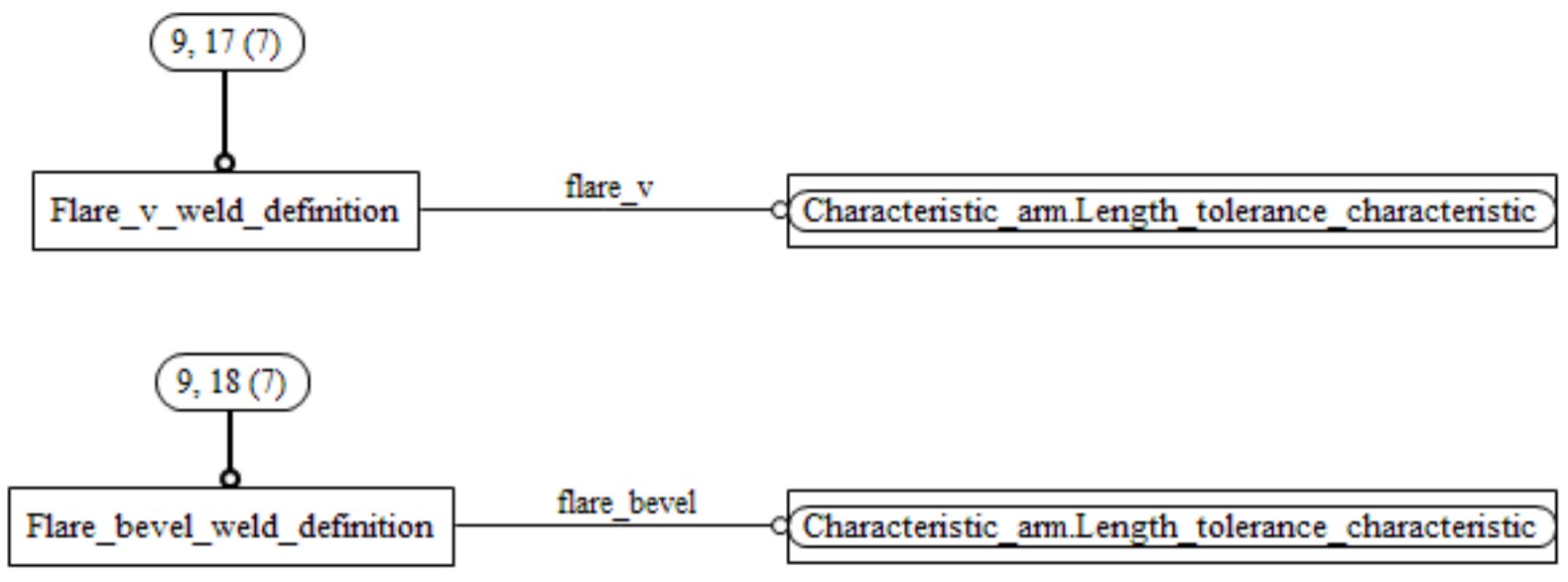

Figure 18 Weld ARM Diagram 7 of 14 


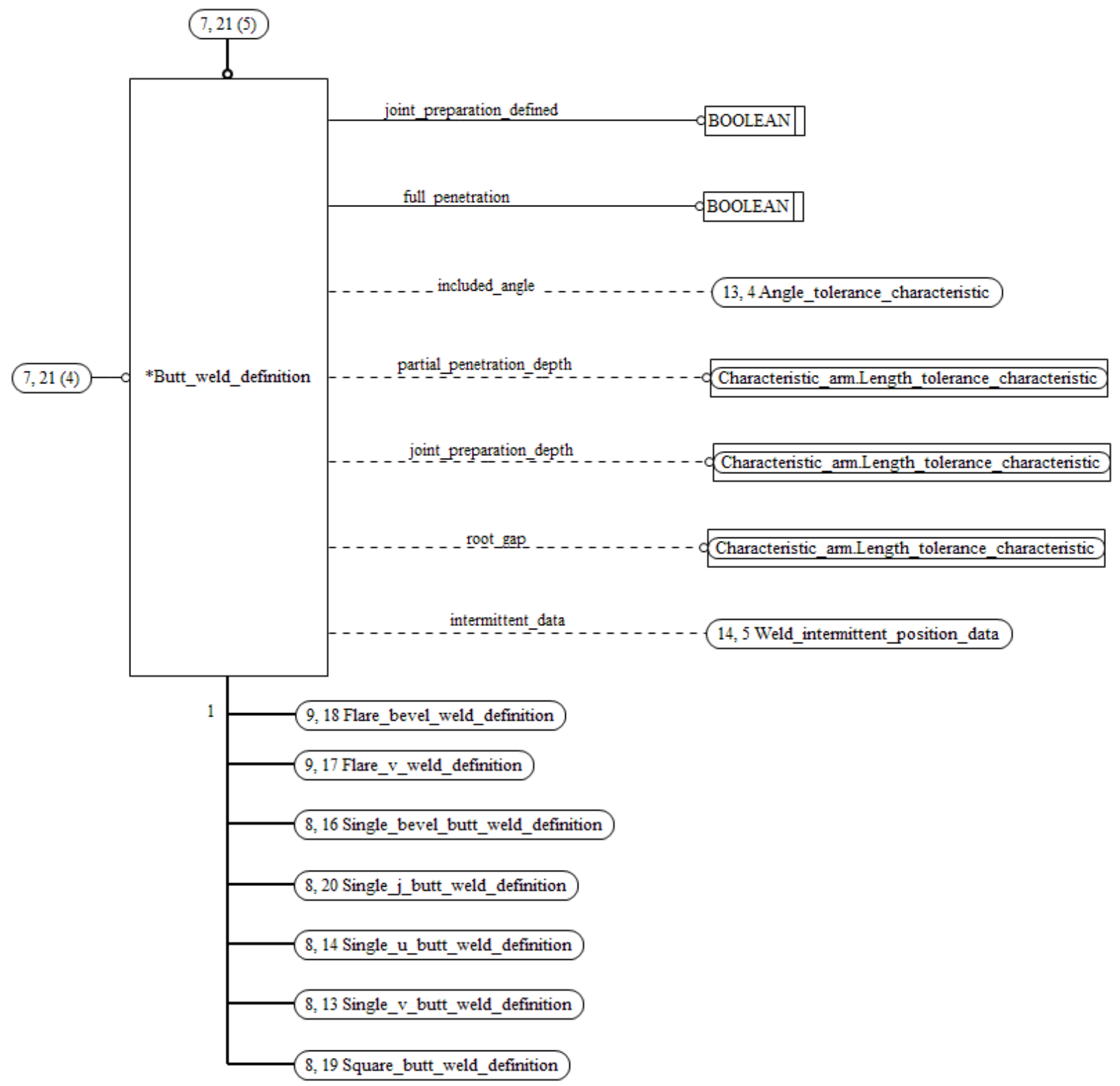

Figure 19 Weld ARM Diagram 8 of 14 

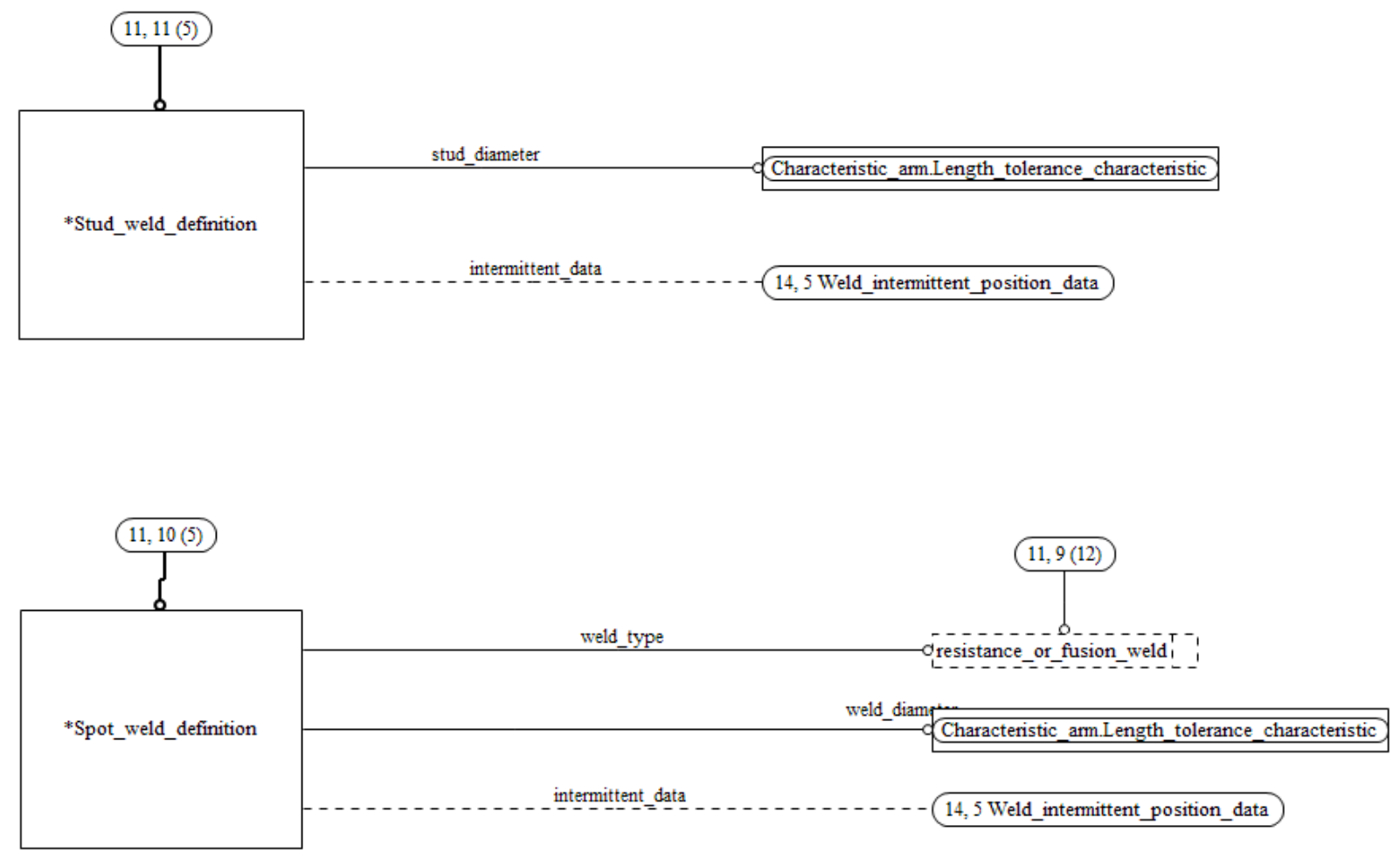

Figure 20 Weld ARM Diagram 9 of 14 


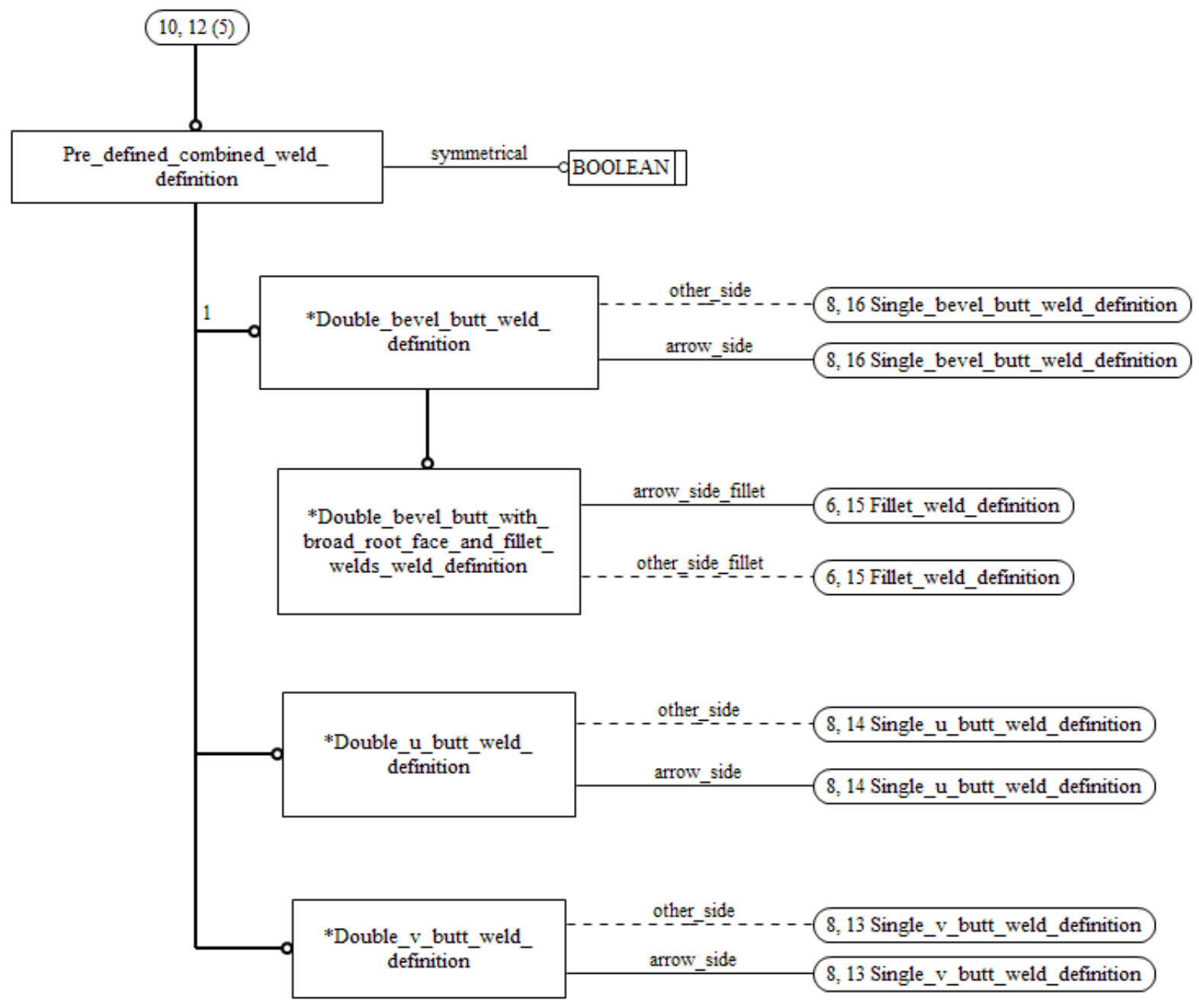

Figure 21 Weld ARM Diagram 10 of 14 

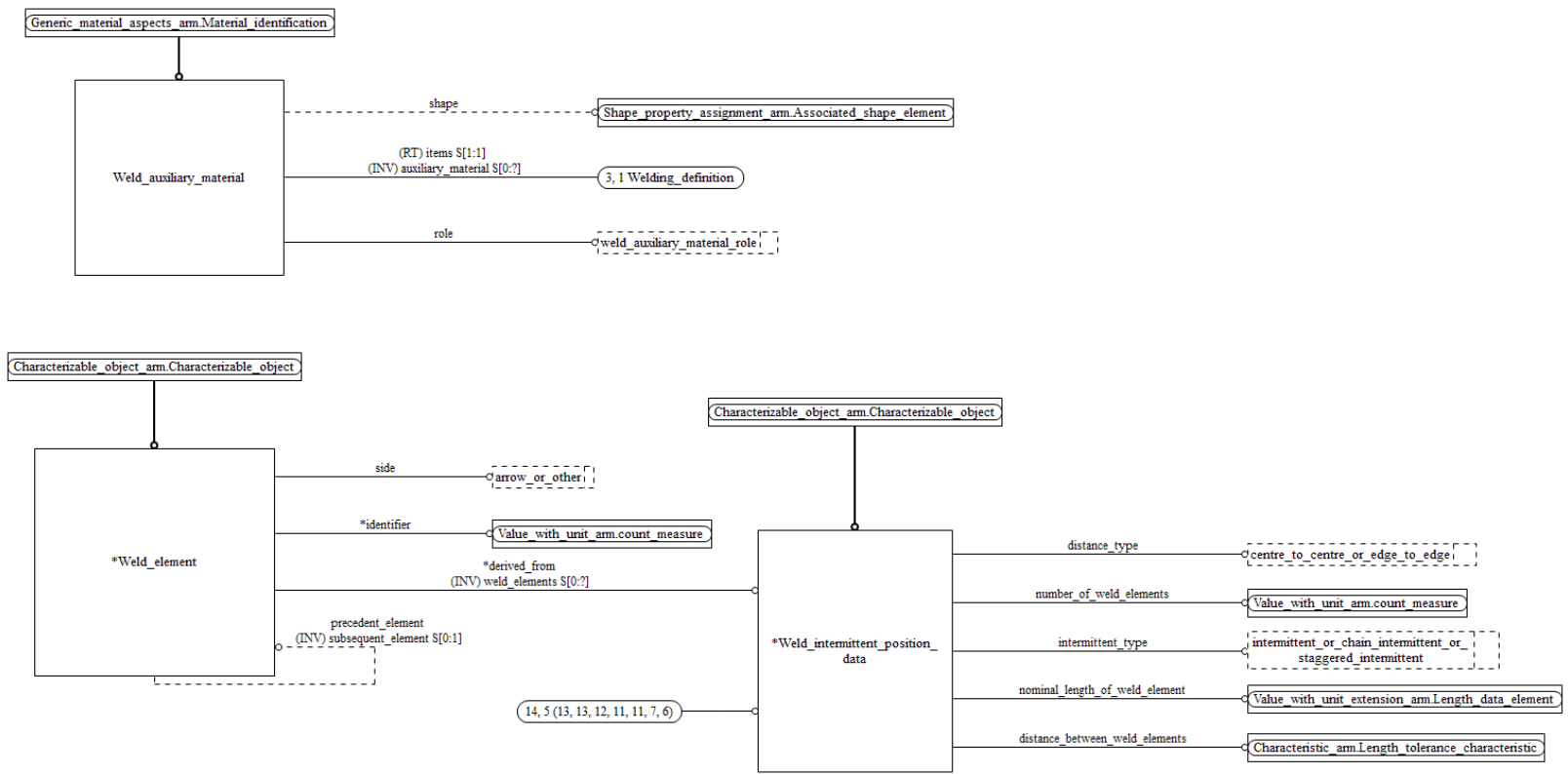

Figure 22 Weld ARM Diagram 11 of 14 

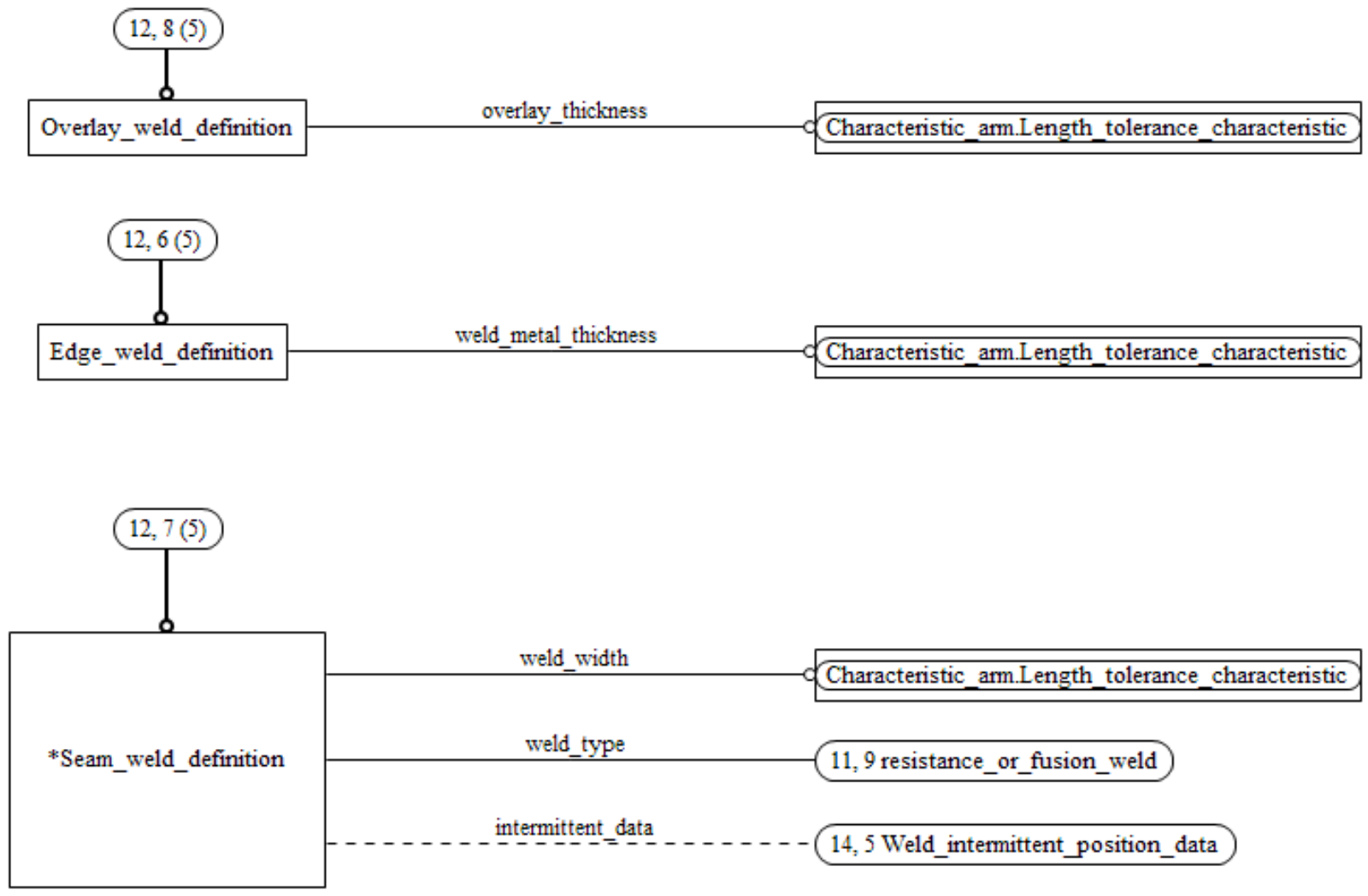

Figure 23 Weld ARM Diagram 12 of 14 

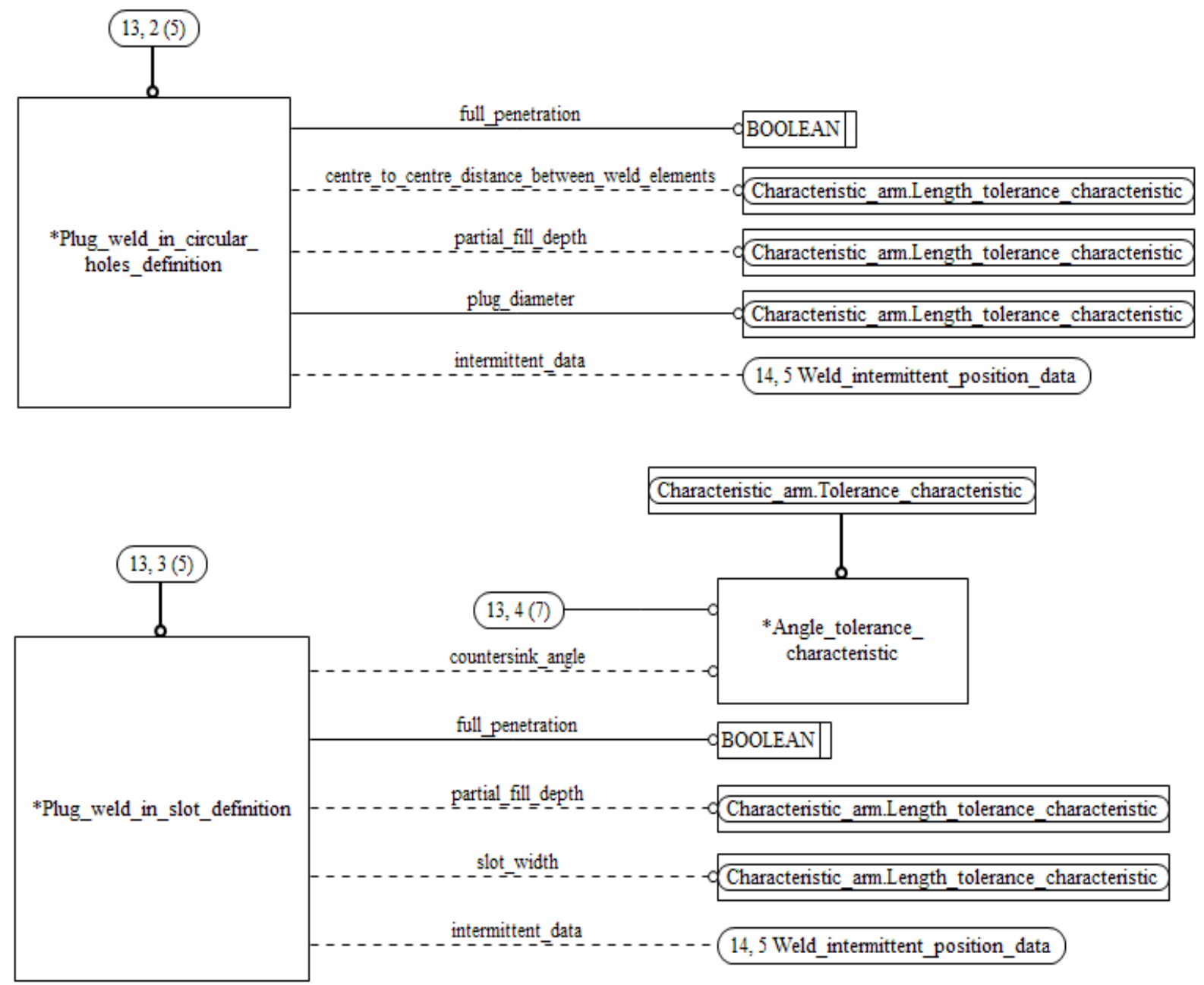

Figure 24 Weld ARM Diagram 13 of 14 

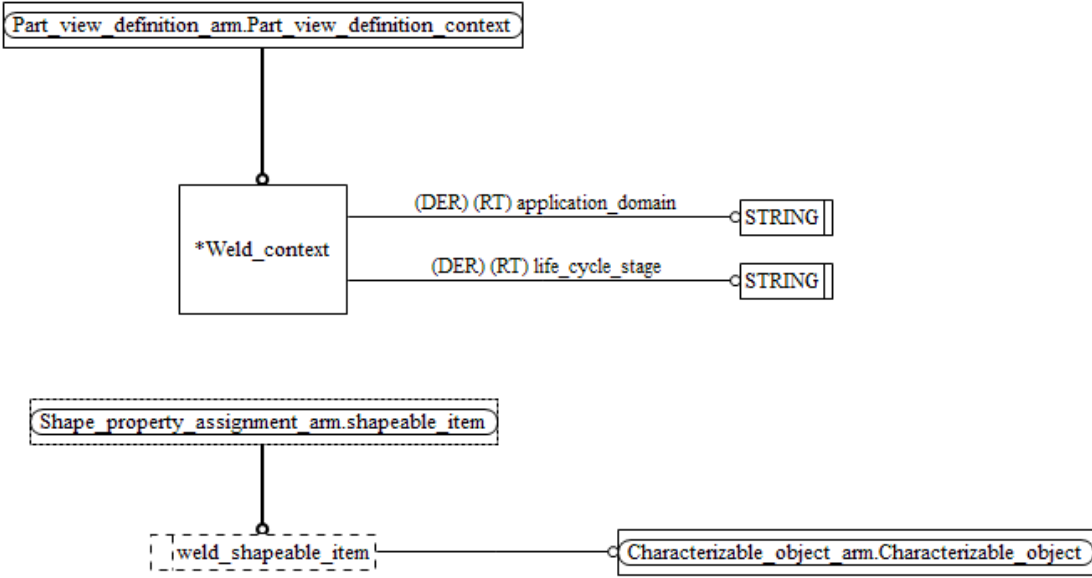

Dimension_tolerance_arm.dimension_target

weld dimension target

Model_based_3d_geometrical_dimensioning_and_tolerancing_representation_arm.Nominal_3d_feature

Figure 25 Weld ARM Diagram 14 of 14 


\section{concluding remarks}

New ARM EXPRESS for Screw thread and for Weld are proposed for STEP. The EXPRESS models were integrated using STEPmod[11] architecture instantiated in[12] into a long form EXPRESS model for evaluation. It is anticipated that further testing and validation of the Screw thread and Weld proposals will occur in the CAX-IF and LOTAR PMI projects.

\section{Acknowledgements}

The contribution of the AP 242 edition 1 project in providing source data in bugzilla that was used in the creation of this document is acknowledged. The contribution of the LOTAR PMI team in providing valuable subject matter expertise for welds and screw threads is acknowledged. The contribution of Kevin LeTutor and Animata Mbengue for EXPRESS-G diagram preparation is acknowledged. 
Works cited:

1. Kemmerer S. STEP the Grand Experience, NIST Special Publication 939. Washington, DC.: US Government Printing Office; 1999

2. ISO 10303-242:2014: Industrial automation systems and integration -- Product data representation and exchange -- Part 242: Application protocol: Managed Model-based 3D Engineering.

3. ISO 1101: Geometrical product specifications (GPS) -- Geometrical tolerancing -- Tolerances of form, orientation, location and run-out.

4. $\quad$ AWS A2.4:2012 Standard Symbols for Welding, Brazing and Nondestructive Examination.

5. ISO 2553:2013: Welding and allied processes-- Symbolic representation on drawings -- Welded joints.

6. Society of Automotive Engineers. Screw threads : UNJ profile, inch controlled radius root with increased minor diameter. [Warrendale Pa.]: SAE International; 2004

7. ISO 3161:1999 Aerospace - UNJ threads - General requirements and limit dimensions.

8. ISO 5855-1:1999 Aerospace - MJ threads - General requirements.

9. Feeney AB, Thurman TR. Introducing a New ISO Standard for Exchanging Product Manufacturing Information. to be published.

10. ISO 10303-11 Industrial automation systems and integration-Product data representation and exchange-Part 11: Description methods: The EXPRESS language reference manual. 2004

11. Feeney AB. The STEP modular architecture. Journal of Computing and Information Science in Engineering(Transactions of the ASME). 2002;2:132-135.

12. STEP Module and Resource Library (SMRL) version 6. 2014

13. LOTAR. www.lotar.org

14. SAE AS8879 Aerospace standard - UNJ Profile, Inch Controlled Radius Root with Increased Minor Diameter.

15. ISO/TS 10303-442: Industrial automation systems and integration -- Product data representation and exchange -- Part 442: Managed model based 3d engineering.

16. ISO/TS 10303-1026: Industrial automation systems and integration -- Product data representation and exchange -- Part 1026: Application module: Assembly structure.

17. Hamilton MH, Sullivan GR. Elements of a welding symbol. 2011

18. ISO/TS 10303-1649: Industrial automation systems and integration -- Product data representation and exchange -- Part 1649: Application module: Assembly technology. 19. ISO/TS 10303-1814: Industrial automation systems and integration -- Product data representation and exchange -- Part 1814: Application module: Machining features.

20. ISO/TS 10303-1051: Industrial automation systems and integration -- Product data representation and exchange -- Part 1051: Application module: Geometric tolerance. 
21. ISO/TS 10303-1050: Industrial automation systems and integration -- Product data representation and exchange -- Part 1050: Application module: Dimension tolerance.

22. ISO/TS 10303-1816: Industrial automation systems and integration -- Product data representation and exchange -- Part 1816: Application module: Model based 3d geometrical dimensioning and tolerancing representation.

23. ISO/TS 10303-1032: Industrial automation systems and integration -- Product data representation and exchange -- Part 1032: Application module: Shape property assignment.

24. ISO/TS 10303-1366: Industrial automation systems and integration -- Product data representation and exchange -- Part 1366: Application module: Tagged_text_representation. 25. ISO/TS 10303-1761: Industrial automation systems and integration -- Product data representation and exchange -- Part 1761: Application module: Information product.

26. ISO 10303-110: Industrial automation systems and integration -- Product data representation and exchange -- Part 1110: Application module: Surface conditions. 27. ISO 10303-224: Industrial automation systems and integration -- Product data representation and exchange -- Part 224: Application protocol: Mechanical product definition for process planning using machining features.

28. ISO 10303-238: Industrial automation systems and integration -- Product data representation and exchange -- Part 238: Application protocol: Application interpreted model for computerized numerical controllers.

29. ISO 14649-10 Industrial automation systems and integration -- Physical device control Data model for computerized numerical controllers -

Part 10: General process data.

30. ISO 10303-214: Industrial automation systems and integration -- Product data representation and exchange -- Part 214: Application protocol: Core data for automotive mechanical design processes.

31. Pratt MJ, Goult RJ. Analysis of Feature Definitions in Edition 2 of AP203 and in AP224 of ISO 10303. 2008;N8538

32. Thurman TR, Paff E. Review of ISO/TS 10303-1814 Machining features application module and of ISO 10303-522 Machining feature application interpreted construct. 2014 33. ISO 10303-242:2014 Industrial automation systems and integration -- Product data representation and exchange -- Part 242: Application protocol: Managed model based 3d engineering.

34. ISO 10303-41: Industrial automation systems and integration -- Product data representation and exchange -- Part 41: Integrated generic resource: Fundamentals of product description and support.

35. ISO/TS 10303-1671: Industrial automation systems and integration -- Product data representation and exchange -- Part 1671: Application module: Feature and connection zone. 